\title{
Active polymer nanofibers for photonics, electronics, energy generation and micromechanics
}

Luana Persano ${ }^{1,2, *}$, Andrea Camposeo ${ }^{1,2, *}$ and Dario Pisignano ${ }^{1,3, *}$

${ }^{1}$ National Nanotechnology Laboratory of Istituto Nanoscienze-CNR, via Arnesano, I-73100 Lecce (Italy)

${ }^{2}$ Soft Materials and Technologies S.r.l., Via Leuca, I-73020, Cavallino (Italy)

${ }^{3}$ Dipartimento di Matematica e Fisica “Ennio De Giorgi”, Università del Salento, via Arnesano I73100 Lecce (Italy)

*E-mail addresses: luana.persano@nano.cnr.it, andrea.camposeo@nano.cnr.it, dario.pisignano@unisalento.it; Phone number: +39 0832298104.

\begin{abstract}
Active polymer nanofibers for opto- and nano-electronics benefit from low cost and versatile fabrication processes and exhibit an unequalled flexibility in terms of chemical composition, physical properties and achievable functionality. For these reasons, they have rapidly emerged as powerful tool for nanotechnologies and as building blocks of a wide range of devices. Both bottom up and top down nanofabrication concepts have been developed to produce nanofibers made of conjugated or other functional polymers and blends. This article summarizes and reviews the chemico-physical and functional requirements for polymer nanofibers to be used in opto- and nanoelectronics, as well as recent advances in various promising device architectures, such as light emitting and photovoltaic devices, photodetectors, field-effect transistors, piezo- and thermoelectric generators, and actuators. The outlook of functional polymer nanofibers and of devices based on them is also outlined and discussed.
\end{abstract}

Keywords: Nanoelectronics, Optoelectronics, Energy harvesting, Polymer Nanofibers, Actuators, Electrospinning 
Published in Progress in Polymer Science 43, 48-95, doi: 10.1016/j.progpolymsci.2014.10.001 (2015).

\section{Contents}

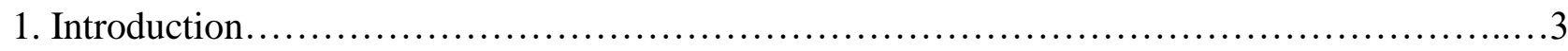

2. Requirements of polymer nanofibers for opto- and nano-electronic applications................6

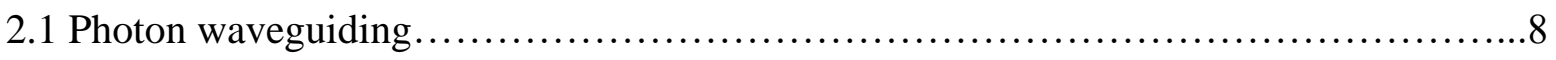

2.2 Photon management.......................................................

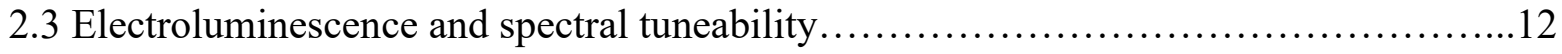

2.4 Charge-carrier transport..........................................................

2.5 Applications in smart textiles and electronic skin.............................. 15

3. Fabrication technologies for active polymer nanofibers ................................ 17

3.1 Self-assembly........................................................ 18

3.2 Templates.................................................................

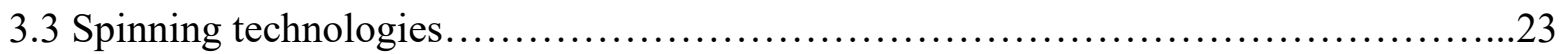

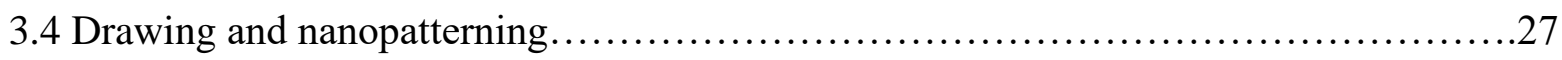

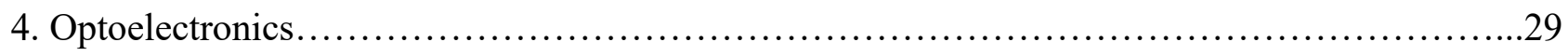

4.1 Light emitting devices and lasers......................................... 30

4.2 Photodetectors and photovoltaics.................................................

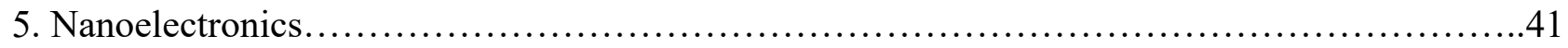

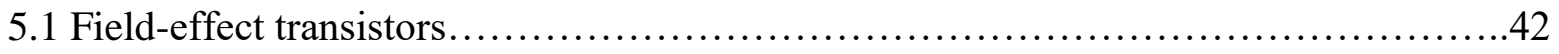

5.2 Energy nano-generators.................................................

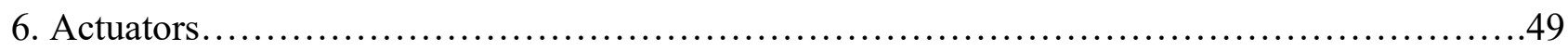

Conclusions and perspectives.................................................... 50

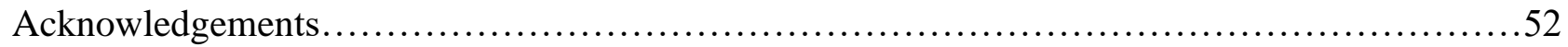

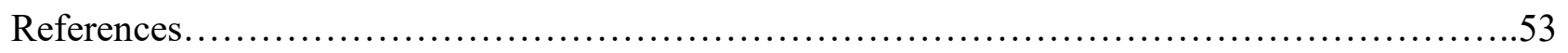

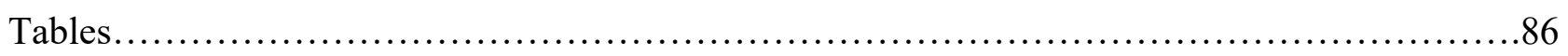


Published in Progress in Polymer Science 43, 48-95, doi: 10.1016/j.progpolymsci.2014.10.001 (2015).

\section{Introduction}

Synthetic and processing technologies to produce, functionalize and assemble polymer nanofibers and materials and devices based on them have achieved enormous advances in the last decade [1, 2, 3]. Indeed, the use of polymer nanofibers for an enormous variety of applications grounds on solid motivations due to their ultra-high surface-to-volume ratio, morphological and optical anisotropy, unique mechanical characteristics [4], and other physico-chemical properties, such as charge transport, thermal conductivity [5], and molecular adsorption capability, which can be significantly enhanced compared to bulk, film-forming, or macroscopic fiber-based materials. These features make polymer nanofibers interesting for all those technological fields where a very large surface is needed in order to allow nanostructures to interact effectively with external liquid or gas media. Applications include the realization of sensors and bio-sensors, the development of most efficient materials for catalysis, filtration, biotechnology, regenerative medicine and tissue engineering. In all these fields, technologies to realize nanofiber-based materials have already reached commercialization, with a lot of dedicated start-up companies [6]. As a matter of fact, some industrial, goods-producing segments, such as those addressing the commercialization of dedicated equipment and of products whose added value is directly provided by nanofibers, are gaining increasing visibility on the market. This is making the field one of the most vibrant in the broad framework of nanotechnologies, due to the actual perspectives of socio-economic impact.

In other areas of applications, polymer nanofibers are still relatively immature as newly developed material. However, a few of these fields, such as nanophotonics and nanoelectronics, are tremendously intriguing from a scientific and technological viewpoint. Here, future, novel materials and devices have being seeded over the last few years, by realizing nanofibers made of optically and electronically active molecules. The classes of materials utilizable to this aim are well-known. On one side, one can consider conjugated polymers, i.e. macromolecules presenting unpaired $(\pi)$ 
Published in Progress in Polymer Science 43, 48-95, doi: 10.1016/j.progpolymsci.2014.10.001 (2015).

electrons. These polymers can exhibit electrical conductivity, together with an either onedimensional or two-dimensional topological structures, as well as various other peculiar physical properties which make them excellent materials for many applications in optoelectronics, nanophotonics and nanoelectronics [7]. Such features comprise semiconducting behavior, wide tuneability of the energy gap between the highest occupied molecular orbital (HOMO) and the lowest unoccupied molecular orbital (LUMO), and at least partially controllable supramolecular ordering and anisotropy of the resulting optical and electronic properties. Nowadays it has become clear that active nanofibers can be realized with conjugated polymers, following a number of complementary fabrication approaches (Table 1). Examples include many derivatives of poly $(p$ phenylene vinylene) (PPV) [8-31], polythiophenes including especially poly(3-alkylthiophene)s (P3ATs) [9, 12, 20, 32-51], poly(fluorene)s [9, 14, 21, 52-62], various side-chain grafted poly(arylene ethynylene)s [63], polyacetylenes [64-66], etc.. The molecular structures of a few of these polymers, relevant in this framework, are shown in Fig. 1.

A different possibility relies on preparing nanocomposite nanofibers, namely blending polymers (even optically and electrically inert) with molecular dopants or nanocrystals as active buildingblocks. This strategy is often preferred when the preparation of nanofibers requires good viscoelastic or thermoplastic behavior from the used polymer solutions or matrices $[9,16,47,48$, 67-72], which conjugated polymers and low-molar-mass molecules do not normally provide. The range of applications where active nanofibers made of conjugated polymers or nanocomposites are exploited is continuously increasing, and include so far organic light-emitting devices (OLEDs), solid-state lasers, sub-wavelength waveguides and other nanophotonic components, photovoltaic cells, transistors and phototransistors, rectifying junctions, logic gates and nonvolatile flash memories.

Furthermore, even some insulating polymers can be of great interest as materials allowing active nanofibers to be fabricated. This is case, for instance, of poly(vinylidene fluoride) (PVDF) and its derivatives. In the last few years, these polymers have been employed to produce piezoelectric 
Published in Progress in Polymer Science 43, 48-95, doi: 10.1016/j.progpolymsci.2014.10.001 (2015).

nanofibers for energy harvesting through nanogenerators and for pressure sensing and accelerometry. Recently, not only piezo-polymers, but also conductive polymer materials and fibers with good thermoelectric properties have begun to be used for the realization of miniaturized components for energy generation, a perspective of remarkable interest for the development of distributed sources of renewable electricity recovering waste heat. Finally, the production of actuators exploiting the elongated and robust geometry of polymer nanofibers is another intriguing prospect, enabling the development of novel, effective and cheap elements for robotics and biotechnologies operating at different length-scales.

This review presents active polymer nanofibers for application in nanophotonics and nanoelectronics, summarizing the main technologies reported to date and allowing these nanostructures to be fabricated, and the main fields of use and proposed device platforms for the resulting materials. Rigorously speaking, by 'nanofibers' one should mean strongly elongated structures, in which the lateral size (diameter) are strictly smaller than $100 \mathrm{~nm}$. However, in current literature, a broader and less rigorous use of the word 'nanofiber' can be often found. In this case, 'nano' is frequently employed as ample synonym of 'miniaturized', 'extremely small' objects, and used to label fibers with lateral size below, or around the scale of $1 \mu \mathrm{m}$. On one side, this is partially justified due to the quite broad dispersion in size obtainable by various fabrication processes, leading to samples and mats where filaments having smaller diameter are present together with bigger fibers. Due to the importance of these structures for most of applications, here we will also include materials, techniques, and devices potentially involving fibers with diameter above $100 \mathrm{~nm}$, thus providing a more complete and wider view on recent progresses in the field.

Our aim is substantially different compared to other outstanding reviews already published on polymer nanofibers $[3,73-76]$. While synthetic and processing details for the various classes of active polymer nanofibers can be found in the original publications, our focus will be in highlighting the broad strategy at the base of recently reported developments. To this aim, the first 
Published in Progress in Polymer Science 43, 48-95, doi: 10.1016/j.progpolymsci.2014.10.001 (2015).

effort is addressed to sketching the desired features of electrically-active and light-emitting nanofibers, namely the requirements that these nanostructures have to fulfill in order to present a clear added value for next-generation photonics and electronics. Then, we proceed to review the recent achievements reported in the literature in this respect. What are the desired specifications for organic nanofibers to be useful in optoelectronics? Which performances or physical properties does one expect? Are clear and effective experimental routes available, in terms of material design and polymer processing, in order to approach or meet these specifications? We believe that a clear review at hand which addresses these issues can be helpful given the current stage of development of research on these polymer nanomaterials applied to the fields of nanophotonics and nanoelectronics. For these reasons, presenting those issues and answering the questions above, introducing prototype material design solutions which have been proposed to this aim, is the scope of the next Section (Sec. 2). In order to achieve the desired characteristics of nanofiber-based materials, a crucial role is played by polymer synthesis and processing methods which allow nanostructures to be produced. These technologies include self-assembly, synthetic or growth approaches involving either hard or so-called soft templates, various spinning techniques assisted by flows or by external fields, and even top-down drawing. The advancements in all these technologies are summarized in Sec. 3. Sections 4, 5 and 6 then present progresses in devicemaking methods and in technological platforms allowing active polymer nanofibers to be embedded in components as well as in more complex architectures for nanophotonics and nanoelectronics. The second peculiar feature of this work is of emphasizing research as recent as possible, with a clear focus on the last decade according to the spirit of reviews in Progress in Polymer Science.

\section{Requirements of polymer nanofibers for opto- and nano-electronic applications}

First of all, when designing polymer nanofibers for opto- and nano-electronics, what one does not usually look for are quantum confinement effects. In organic semiconductors, one normally has 
Published in Progress in Polymer Science 43, 48-95, doi: 10.1016/j.progpolymsci.2014.10.001 (2015).

Frenkel excitons with large binding energy and Bohr radius $\leq 1 \mathrm{~nm}$, which makes very hard confining them in nanostructures. Active polymer nanofibers are no exception in this respect. One should also point out that, although several demonstrations exist about soft nanolithography and spinning for producing $1 \mathrm{~nm}$-scale elongated features and fibers made of various resists, elastomers, and thermoplastic materials [78-80], reaching spatial resolutions below $10 \mathrm{~nm}$ in conjugated polymers has been proven to be much more difficult, due to issues related to the relatively lower viscoelasticity and solubility, formation of aggregates, oxidation making them susceptible at high temperatures, etc.

Instead, the geometry of nanofibers has rendered them the most intriguing nanostructures to allow photons to be managed and addressed within optical circuits, and to allow electrodes of nanoelectronic devices to be bridged. However, each particular application has its own specific requirements in terms of physical properties of its building blocks. Besides fulfilling such requirements, the main question when designing active polymer nanofibers in most recent literature has been if these nanostructures are able to outperform the corresponding bulk materials or thin films, thus becoming appealing for next-generation organic photonics and electronics. The answer to such question has been, most of times, positive.

For example, in photonic circuits nanofibers have been used to perform different functions. The multi-functional capability of these nanostructures, depending on the internal composition and molecular orientation while the elongated shape and other geometrical characteristics are kept unchanged, is without doubts an important point of strength. First, polymer nanofibers have been extensively employed as waveguides for carrying photons. Second, they could embed additional molecular components to provide the circuits with extra functional blocks along the path of light. Potential dopants include strongly adsorbing elements acting as polarizers, which is the case of embedded oriented molecules, or working as components of mode-locked fiber lasers, which is the case of so-called saturable adsorbers such as carbon nanotubes and graphene [81]. Similarly, active 
Published in Progress in Polymer Science 43, 48-95, doi: 10.1016/j.progpolymsci.2014.10.001 (2015).

compounds can be used in nanofibers which are suitably pumped to amplify light passing through them, thus showing optical gain, which is the case of organic nanofiber lasers (Sec. 4.1). These advancements will be reviewed in Section 4.

Similar considerations about multi-functionality have strongly motivated research on conductive or piezoelectric polymer nanofibers, since these systems can provide nanoelectronic components and circuits with enhanced miniaturization, improved charge-carrier or energy-harvesting properties, and easier interfacing with sensing platforms and soft robotics. The related advancements will be reviewed in Sections 5 and 6. In the following paragraphs (2.1-2.5), we focus instead on the desired specifications and properties of polymer nanofibers, and on the strategies already proposed in order to meet these requirements.

\subsection{Photon waveguiding}

In order to realize effective waveguides, one needs the refractive index, $n$, of polymer nanofibers to be larger than those of the surrounding media, which usually are air $(n \cong 1)$, additional polymer claddings incorporating the fibers $(n \cong 1.3-1.5)$, or the substrate materials on which fibers are deposited [ $n \cong 1.4-1.5$ for poly(methyl methacrylate), glass or silica]. Unfortunately, the refractive indexes of organics are generally quite low (frequently near 1.5), which poses some limitations to the actual waveguiding capability obtained in polymer nanofibers deposited on substrates. For this reason, among the various strategies developed in order to improve light confinement in polymer nanofiber waveguides, the first has been using resists which show relatively higher $n$ values. An example is the SU8 resist (MicroChem Corp.), which has a refractive index around 1.6 in the visible and show good mechanical stability [82]. In addition, doping polymers with inorganic nanoparticles such as quantum dots has allowed the effective refractive index along the fiber length to be further increased [82]. The resulting organic/inorganic nanofibers may be used as suspended waveguides, and exploit the quantum dots as light-emitting building blocks. Drawn polyacrylamide and 
Published in Progress in Polymer Science 43, 48-95, doi: 10.1016/j.progpolymsci.2014.10.001 (2015).

polystyrene are other matrices which are interesting in this framework, showing $n \cong 1.6$, being easy

to dope with quantum dots, and possibly showing an increase of refractive index following electronbeam irradiation as shown by the Tong group [83-85]. Some examples of these waveguiding and light-emitting nanofibers are displayed in Fig. 2a-f. It is very likely that methods in which active or inert polymers are mixed together with inorganic light-emitting nanoparticles will be further developed in the near future, due to their unequalled versatility in terms of material composition and achievable photonic properties. An alternative offering good $n$ values (up to about 2.1 in the visible spectral range) are conjugated polymers such as poly(9,9-dioctylfluorene) (PFO) [53] and poly[(9,9-dioctylfluorenyl-2,7-diyl)-co-( $N, N$-diphenyl)- $N, N$-di( $p$-butyl-

oxy-phenyl)-1,4-diaminobenzene)] [86], whose active nanowires have been demonstrated to guide internally emitted photons (Fig. 2g,h). Finally, other approaches could be interesting in view of tailoring the $n$ values in nanofibers. For example, the refractive index, together with other properties including the absorption spectrum and water wettability, can be optically controlled by means of photochromic molecules embedded in the polymer host constituting nanofibers or nanotubes [87, 88].

Other physical properties, which have been demonstrated to strongly affect waveguiding, are the Stokes shift between the absorption and the emission spectra of the active molecules in lightemitting nanofibers, and, for all the species of fibers (both active and passive), their morphology, both in terms of internal heterogeneity, uniformity of the fiber diameter and defects, and in terms of surface roughness. All these aspects directly influence optical losses, namely determine the amount of light which is ultimately transmitted along the nanofibers. It is clear that molecular systems with larger Stokes shift ( $\geq$ a few hundreds of $\mathrm{meV}$ ) are to be preferred as dopants in light-emitting polymer waveguides, because they feature lower self-absorption of emitted light and hence lower optical losses. Reduced self-absorption is very useful also to obtain lasing action from polymer nanofibers showing stimulated emission. In addition, allowing researchers to easily decouple 
Published in Progress in Polymer Science 43, 48-95, doi: 10.1016/j.progpolymsci.2014.10.001 (2015).

excitation and luminescence signals, large Stokes shifts also facilitate the experimental investigation of the optical and emission properties of polymer nanofibers, as carried out in many studies recalled later in this review. In many works, a very effective way to increase the Stokes shift of a light-

emitting system has been using blends in which the excitation is transferred from a high-energy host to a low-energy dopant. The latter then emits photons at a wavelength which is higher than those at which the host absorbs. These photons are therefore unlikely to be re-absorbed by the donor molecules, which constitute the most of the active medium. Sec. 2.3 will review some of these systems in the context of light-emitting polymer nanofibers.

\subsection{Photon management}

Photon coupling is another issue, since injecting light from external optics or free space into polymer nanofibers is always complex, especially if nanofibers have sub-wavelength diameter. In this respect, waveguiding excitation has been proposed as effective approach in order to obtain enhanced excitation efficiency in light emitting nanofibers, as well as improved photostability of the embedded chromophores [84]. The setup for waveguiding excitation in nanofibers, supported on a substrate with low refractive index such as $\mathrm{MgF}_{2}$, typically includes micromanipulators to handle a fiber taper and to couple evanescent light with nanofibers, and a fluorescence microscope equipped with a long working-distance objective.

More in general, a proper management of light in individual polymer nanofibers and in optical circuits composed of them is crucial because photons can work as carriers of information, induce non-linear response and optical gain in dyes and conjugated polymers at given sites, and so on. For all the applications above, however, light-scattering occurring within polymer fibers is a relevant issue, therefore dedicated efforts have been performed to investigate and possibly minimize scattering sources. In most of cases, in active polymer nanofibers light-scattering has been observed in the so-called Rayleigh regime, namely it originates from scattering centers which have size much smaller than the light wavelength [53]. These scattering centers have been ascribed to internal 
Published in Progress in Polymer Science 43, 48-95, doi: 10.1016/j.progpolymsci.2014.10.001 (2015).

crystallites or more in general to density or compositional inhomogeneities which can be distributed along the fiber length, and are critically related to the details of fiber production processes.

Consequently, achieving an effective management of photons in or by light-emitting nanofibers is extremely demanding from the point of view of nanofabrication technologies.

Another requirement for many applications (particularly for realizing excitation sources whose signal can be easily decoupled from the excited one [13], as needed in high-sensitivity diagnostics and in fluorescence microscopy) is the availability of polarized light emission. This is a field where the added value of photon sources based on polymer nanofibers is particularly relevant. Being made of molecules, and consequently embedding transition dipoles, which are on average much better aligned compared to films or bulk materials, polymer nanofibers have been found to generally exhibit superior polarization of emitted light. Furthermore, several techniques have allowed nanofibers to be mutually aligned and organized within arrays, which in turn has allowed polarized emission to be collected from macroscopic areas (usually up to the scale of $\mathrm{cm}^{2}$ ). These aspects thus make polymer nanofiber light sources easy to couple with other large-area platforms including biotechnologies and smart textiles. In particular, the polarization performance of nanofibers can be measured by the polarization ratio, $\chi$, which is given by the ratio of the intensity of light polarized along the fiber longitudinal direction $\left(I_{Z}\right)$ to the intensity of light polarized along a perpendicular direction $\left(I_{X}\right)$. Many papers also report on the so-called polarization anisotropy, $A_{\chi}=\left(I_{Z}-I_{x}\right) /\left(I_{Z}+2\right.$ $I_{X}$ ). An example of how the polarized emission from conjugated polymer nanofibers is modulated by varying the angle between the fiber longitudinal axis and the axis of a polarizer is shown in Fig. 3a. Overall, the $\chi$ and the $A_{\chi}$ values demonstrated so far are quite scattered, and strongly depend not only on the used polymer species, but also on the fabrication technology used to produce nanofibers and on the specific processing details. The conjugated polymer, poly[2-methoxy-5-(2'ethylhexyloxy)-1,4-phenylenevinylene] (MEH-PPV), is an interesting example in this respect (Fig. $3 b)$. A polarization ratio of about 2, and between 2.2 and 13 has been reported for nanofibers 
Published in Progress in Polymer Science 43, 48-95, doi: 10.1016/j.progpolymsci.2014.10.001 (2015).

realized by nanofluidic lithography [21] and by electrospinning [13, 15, 71], respectively. In fact, using composites in which MEH-PPV has been blended with optically inert and more processable polymers, such as polyethyleneoxide (PEO) $[17,18,20]$ and polycaprolactone, appears a promising strategy to increase the polarization ratio in these electrospun nanofibers $[15,71]$. Indeed, isolating conjugated polymer molecules within the host matrix, these blends have been supposed to suppress the interchain energy transfer mechanisms which are detrimental to polarized light emission [15]. Interestingly, values of the polarization ratio around 12 have also been achieved by a completely different method, namely orienting MEH-PPV in aligned mesoporous silica films [89]. Other polymers exhibit a similarly various phenomenology. For instance, PFO nanowires synthetized by molding in membrane templates (Sec. 3.2) reach $\chi=4.0$, outperforming electrospun nanofibers [57, 59, 90]. Electrospun nanofibers of poly[(9,9-dioctylfluorenyl-2,7-diyl)-co-(1,4-benzo-\{2,1'-3\}thiadiazole)] reach $\chi=5$ and $A_{\chi}=0.6$, but only upon properly choosing the processing solvents [91]. A few exotic systems have also been studied, such as poly[1-(trimethylsilyl)phenyl-2phenylacetylene] and polymers based on $\mathrm{N}$-alkyl-2,7-di(2-thienyl)carbazoles, in which the prevalent polarization of emitted light is perpendicular to the fiber length $[92,93]$. Finally, one should point out that, following fabrication, light-emitting nanofibers can be stretched to improve molecular alignment in them and consequently further increase the achievable polarization ratio [71]. In general, the investigation of polarization properties and of their time-resolved behavior following excitation is very useful to achieve information on the molecular assembly and excitonic dynamics in polymer nanofibers [94].

\subsection{Electroluminescence and spectral tuneability}

The prerequisite for realizing OLEDs based on active polymer nanofibers is the achievement of nanostructures which both emit bright light and present good charge transport. Advancements related to the latter aspect will be reviewed in the next sub-Section (2.4) and in Section 5. 
Published in Progress in Polymer Science 43, 48-95, doi: 10.1016/j.progpolymsci.2014.10.001 (2015).

Furthermore, all the applications ultimately enabled by polymer electroluminescence require an effective and broad tuneability of the emission color. For this reason, blending different molecular components in order to exploit processes of excitation transfer to tune the emission spectra has been traditionally one of the most active fields of research in organic optoelectronics. Fortunately, almost all the techniques allowing polymer nanofibers to be realized work with blends as well. By varying the relative concentration of the components, the emission of individual nanostructures and of mats composed of them have been effectively tuned throughout the visible range, obtaining even white emission with very good color purity as measured by the Commission Internationale de l'Eclairage (CIE) coordinates [95-97]. A straightforward approach using mats of nanofibers has been based on incorporating different dyes in each spatially separated nanostructure, thus largely reducing energy transfer phenomena and enabling a direct control of the resulting color emitted by the mats as shown in Fig. 4 [98]. More in general, how the physics of excitation transfer may vary due to the peculiar supramolecular organization inside polymer nanofibers has been very rarely explored. For example, an interesting blend to produce nanofibers can be made by soluble derivatives of PPV, such as the MEH-PPV, and poly(9-vinylcarbazole) (PVK) [10]. Besides color tuning in the resulting fibers, these materials have allowed other interesting effects to be observed. In particular, they undergo an intimate mixing of the different molecular components in fibers as suggested by the observed blue-shift of the emission from MEH-PPV, which indicates reduced aggregation and interchain interaction. The energy transfer from PVK to MEH-PPV is correspondingly more efficient in fibers than in reference films. An emission tuning from blue to green has been also found in electrospun non-wovens made of ternary blends of PFO, poly(2,3-dibutoxy-1,4-phenylene vinylene) and poly(methyl methacrylate) [99].

In addition, an effective color tuning can be obtained also by nanocomposite nanofibers exploiting compounds with very different molecular weights, for instance embedding compounds based on $\mathrm{Tb}$, Er or Re within suitable polymers, such as the polyvinyl alcohol (PVA) [100] or polyvinylpyrrolidone [101], or by incorporating either in-situ synthetized or colloidal quantum dots 
Published in Progress in Polymer Science 43, 48-95, doi: 10.1016/j.progpolymsci.2014.10.001 (2015).

[102-104], or dye-loaded zeolite L crystals [105, 106]. For instance, blending poly[p-xylene- $\alpha$ (dialkyl-sulfonium halide)], which is the sulfonium polyelectrolyte precursor of PPV, with PVA and nanocrystals, has allowed white emission to be obtained by electrospun nanofibers, with an important stabilizing role played by the polymer matrix [103]. Furthermore, using three or more components in organic nanofibers may allow cascades of multiple energy transfer processes to be observed, in which excitations migrate from the compounds with highest energy to those with lowest energy, thus further increasing the possibilities in terms of material design and ultimate spectral tuneability [107]. Finally, a tight control of the color of the emitted light can be achieved by using coaxial nanowire geometries as shown in Fig. 5 [108]. In this way, different radial regions of individual nanostructures present different chromophores, which allows a fine tuning of the spectral emission to be obtained.

\subsection{Charge-carrier transport}

Conductive polymer nanofibers have been increasingly studied in the last years, due to their potential utility as active building blocks to investigate the fundamental transport properties in organic nanostructures, and to their effective embedment in various electronic devices and architectures [76]. A few of such devices will be reviewed in Sec. 5.1. High charge-carrier mobilities and good coupling with electrodes and with dielectric interfaces are generally crucial requirements for these applications. Indeed, achieving high values of the charge mobility is very important both for improving performances of transistors and other electronic components, and for increasing the operation speed of organic-based photodetectors up to well above the MHz regime. Fortunately, a number of reports have demonstrated that polymer nanofibers, due to their frequently more ordered internal supramolecular organization and better molecular orientation, allow researchers to outperform the charge mobility of bulk organic materials and thin films. These results will be reviewed in Sec. 5.1 as well. However, not only pristine conjugated polymer nanofibers, but 
Published in Progress in Polymer Science 43, 48-95, doi: 10.1016/j.progpolymsci.2014.10.001 (2015).

also nanocomposite nanostructures and materials based on nanofibers have been employed to this aim. In addition, in order to realize nanocomposites, active polymer nanofibers can be used not only as host but also as fillers, dispersing the nanostructures within suitable, thermoplastic or thermosetting matrices. For example, mixing conductive polymer nanofibers with electrically insulating matrices has led to the achievement of conductive nanocomposites (Fig. 6) [109, 110]. For producing such materials, however, one needs good amounts of nanofibers, which have to reach homogeneous distributions in the host polymer. It is clear here that a very important role is played by filler-host chemical affinity and interfacial interactions, as well as their by their degrees of solubility in the solvents used to prepare the nanocomposites.

\subsection{Applications in smart textiles and electronic skin}

Another emerging class of applications of polymer nanofibers is found in smart textiles. These are highly bendable, conductive or adaptive coatings and wires which can be freestanding, deposited on various flexible surfaces, or even eventually mixed with weavable textile fibers, and that can be used for providing fabrics and clothes with various functionalities. Examples of functions potentially enabled by such materials include electromagnetic shielding, light-emission, energy harvesting through piezoelectricity or other mechanisms, photovoltaic properties, adaptive response to changes of external environment, sensing, antistatic behavior, and so on [29]. In general, polymer nanofibers are particularly advantageous materials for being embedded in smart textiles, through various stretchable and bendable device platforms [111]. For instance, non wovens made by conductive polymer nanofibers can be covered by thermochromic inks to produce color-changing textiles, as demonstrated by Laforgue using a combination of electrospinning, vapour-phase polymerization, and deposition of functional microcapsules (Fig. 7) [112]. These textiles can be decorated by painted logos and letters, and made electrochromic thanks to the heating properties of the nanofiber mats. The main requirement for effective tuneable and adaptive textiles is therefore a fast response, possibly within times of a few seconds. In this respect, it is clear that the 
Published in Progress in Polymer Science 43, 48-95, doi: 10.1016/j.progpolymsci.2014.10.001 (2015).

miniaturization of functional fibers is a promising route to increase the rate at which smart textiles may respond to external stimuli. Nowadays, these and other flexible, polymer-based materials and systems are being proposed as enabling tools for the Internet of Things, in which individual objects will be uniquely identifiable, will be inter-connected, and will interact by means of wireless tagging [113].

Electron skin is another field of use in which polymer nanofibers used in nanoelectronic devices and responsive textiles will be able to provide a clear added value. For example, both deformable field-effect transistors (FETs) based on conductive nanofibers [46], and the piezoelectric nanofibers [114] reviewed in Sec. 5.2, are well suited to build highly-sensitive pressure sensors and other flexible interfaces embeddable in electronic skin, with applications in hybrid and soft robotics. Here mechanical robustness and flexibility, as well as low cost become crucial in order to make smart textiles embedding active polymer nanofibers of actual interest for everyday use. For instance, interesting studies have been focused on the assessment of the wear resistance of electrospun mats of nanofibers, and on its dependence on processing and annealing conditions [115].

Importantly, a considerable advantage of nanofibers for electronic skin and wearable applications comes from their mechanical properties, and particularly for the mechanical stability that the nanofiber shape can confer on active organic materials, at large bending strains and even at large extensions. In particular, together with wavy device design which minimizes stress accumulation [116], preparing nanocomposite films embedding either carbon nanotubes [117] or nanofiber mats is nowadays one of the most important strategies available to realize flexible and stretchable supports and active layers for electronics. Targeted strain values can be very high, and even exceed $100 \%$ [118-120]. In this respect, the curve contour paths of interconnected nanofiber networks embedded in other polymers or organics can be very useful to stabilize the mechanical and conduction performances. Electrospinning can be especially useful as fiber deposition technology in this respect [111]. Examples of device applications relying on polymer nanofiber composites are numerous, and include stretchable transistor active components made of electrospun poly-3- 
Published in Progress in Polymer Science 43, 48-95, doi: 10.1016/j.progpolymsci.2014.10.001 (2015).

hexylthiophene (P3HT)-based filaments [121], stretchable conductive coatings exploiting poly(3,4ethylenedioxythiophene) (PEDOT)-based fibrils obtained by solution processing with surfactants [122], and highly bendable, large area bulk heterojunction films prepared by a cool-and-heat process and usable in organic photovoltaics [123].

\section{Fabrication technologies for active polymer nanofibers}

In addition to the methods summarized in the next sub-sections, other approaches deserve to be mentioned in this framework. In many studies, nanofibers have been directly achieved by polymerization of monomers, as in the well-known chemical oxidation process of polyaniline (PAN) [124-129]. For PAN, even the control of the sense of helical nanofibers has been achieved by using chiral acids as dopants and by copolymerization involving aniline and $N$-methyl aniline [130]. More in general, for PAN and other polymers, interfacial self-assembly of molecules and interfacial polymerization have both allowed active nanofibers to be produced with good throughput, exploiting interfaces (typically between immiscible fluids) as template surfaces. Interfacial approaches are especially useful if one uses amphiphilic building blocks, whose molecules either orient their hydrophilic and hydrophobic regions towards the polar and the unpolar medium, respectively, or form core-shell architectures [131]. In this way these molecules naturally define two-dimensional or one-dimensional fields, in which they can mutually interact to form nanofibers.

Conductive polymer nanofibers formed by interfacial techniques have been also deposited with high precision in form of monolayers on practically any substrate and under ambient processing conditions, by means of a method based on the emulsification of two immiscible liquids, such as water and oil, in a glass container [51]. To this aim, the two fluids together with polymer nanofibers have been agitated to generate a dispersion of water droplets, coated with fibers at their interface with oil. These droplets then rise at the surface of the emulsion and coalesce, thus leading to the formation of a concentration gradient of the covering nanofibers and to a consequent interfacial 
Published in Progress in Polymer Science 43, 48-95, doi: 10.1016/j.progpolymsci.2014.10.001 (2015).

surface tension gradient. This in turn origins a viscous flow spreading the fibers into monolayer coatings over solid surfaces.

\subsection{Self-assembly}

Self-assembling methods have been developed to produce polymer nanofibers through precipitation and eventual crystallization from solutions made by so-called marginal solvents [32, 33, 132, 133]. These are solvents in which organic macromolecules are relatively poorly soluble, or in which the solubility is relatively good only at high temperature. As a consequence, gradually reducing the solution temperature leads to the precipitation of the solid, polymeric content, which can form nanofibers by self-assembly. Of course, several details of the process have been varied. For example, mixtures of different solvents, in which polymer molecules are soluble to a different extent, can serve to obtain solutions priming the self-assembly of nanofibers. Finally, besides engineering the chemistry of solutions, depositing them on substrates can favor self-assembling processes, through the resulting interfacial interactions and solvent evaporation. For this reason, the substrate functionalization and the solvent vapor pressure play here a major role. This has been studied, for instance, in various reports in which active polymer nanofibers have been obtained following solution casting or spin-coating [39-41, 134-137]. A recent development of strategies using increased solvent vapor pressures is the so-called solvent vapor enhanced drop casting method [138]. Here, the cast polymer has been exposed to an atmosphere saturated with solvent vapor. This step can be particularly advantageous to obtain nanofibers out of materials, such as the donor-acceptor cyclopentadithiophene-benzothiadiazole copolymer, uneasy to be processed reliably otherwise. Interestingly, in all these approaches the resulting nanostructures often exhibit a good microcrystalline order, which is of great interest for making them highly conductive.

The case of self-assembled P3HT nanofibers has been the most largely investigated in this respect $[35-37,39,40,94,139,140]$. These nanostructures can reach a length of several micrometers and a width below $10 \mathrm{~nm}$, with a $\pi$-stacking direction which is parallel to the fiber longitudinal axis as 
Published in Progress in Polymer Science 43, 48-95, doi: 10.1016/j.progpolymsci.2014.10.001 (2015).

schematized in Fig. 8. This strongly favors the transport of holes along this direction (Sec. 5). In a study, the fiber width has been found to increase with the polymer molecular weight, saturating at about $15 \mathrm{~nm}$ for a weight of about 10,000 a.m.u., due to chain folding in a plane perpendicular to the fiber longitudinal axis [38]. In addition, the nanofiber diameter has been found to slightly increase also for P3ATs with longer alkyl chains [32, 33]. In another, systematic study on nanofibers made of P3ATs with varying alkyl chain lengths (from 3 to $9 \mathrm{C}$ atoms), for aliphatic and (chlorinated) aromatic hydrocarbon solvents the value of the solvent refractive index has been found to be somehow related to the feasibility of that solvent for inducing the formation of nanofibers [34]. Furthermore, the order achievable within nanofibers has been also found to depend on the alkyl chain length. Recently, a few studies have been focused on the occurrence of $\mathrm{H}$ - and Jaggregate character in self-assembled P3HT nanofibers [140, 141], and have highlighted the possibility of interesting molecular reorganization processes which take place in them, following heating or other processing in solution [142, 143].

The electron-conducting material, poly(benzobisimidazobenzophenanthroline) (BBL), has been studied due to its capability of forming nanobelts in solution [144]. These nanostructures have been achieved following dropwise addition of a methanesulfonic acid solution into a solution made by chloroform and methanol, which work as weakly-interacting solvent and deprotonating base for BBL, respectively (Fig. 9a). This allows the assembling process to be initiated and nanobelts to be formed (Fig. 9b). Interestingly, these nanobelts show an internal organization of the polymer molecules radically different from P3ATs, with a $\pi$-stacking direction perpendicular to the fiber longitudinal axis (Fig. 9c).

Self-assembling methods have been largely developed for block copolymers as well. Regioregular poly(3-butylthiophene)- $b$-poly(3-octylthiophene) in 1,2-dichlorobenzene solutions have been found to self-assemble into crystalline nanowires having a width of $13.5 \mathrm{~nm}$, and length from hundreds of $\mathrm{nm}$ to $1 \mu \mathrm{m}$ [145]. Diblock copolymers of P3HT and poly(methyl acrilate), following chloroform 
Published in Progress in Polymer Science 43, 48-95, doi: 10.1016/j.progpolymsci.2014.10.001 (2015).

solution casting under ambient conditions, self-assemble into complex nanofibrillar structures [41]. Another study has been focused on polythiophene diblock copolymers containing side chains of different polarity, such as hexyl and triethyliene glycol groups which are nonpolar and polar, respectively [146]. In these molecules, polar groups allow a strong solubility contrast to be obtained. As a consequence, following addition of methanol to chloroform solutions, nanowires are formed, and in presence of potassium ions during crystallization, helical fibers are obtained which assemble into superhelices (Fig. 10). Conjugated, amphiphilic block copolymers of polythiophene and polyethylene glycol have been found to form different superstructures composed of bundled and branched fibers, following their self-assembling with preformed polythiophene nanofibers and depending on the used solvents and on the specific chain length and species [147]. The triblock copolymer, poly(ferrocenyldimethylsilane- $b$-2-vinylpyridine- $b$-2,5-di-(2'-ethylhexyloxy)-1,4phenylvinylene), forms cylindrical micelles in 2-propanol solution [148]. These micelles can be fragmented by sonication, ultimately resulting in fiber-like micelles following the addition of extra diblock polymer, poly(ferrocenyldimethylsilane- $b$-2-vinylpyridine, in the solution. Iteratively repeated, this process so allows multiblock micelles to be produced, which present alternating lightemitting compartments corresponding to the presence of di-(2'-ethylhexyloxy)-1,4-phenylvinylene) moieties. In this way fluorescent blocks can be used to implement bar codes in polymer lightemitting nanofibers. So-called crystallization-driven self-assembly, which exploits seed micelles as initiators, has been also developed for obtaining cylindrical micelles of controlled length by P3HT$b$-poly(dimethylsiloxane) (P3HT- $b$-PDMS) [149].

Overall, the advantages of self-assembly methods stand in the very high flexibility offered by chemistry in terms of usable molecules and solvents, and in their easiness, cheapness, and relatively good throughput. So far, the main limitation has been related to the difficulty in handling the resulting structures, whose manipulation techniques are still poorly developed, in separating them down to the scale of individual nanofibers, and in effectively addressing single fibers to interface 
Published in Progress in Polymer Science 43, 48-95, doi: 10.1016/j.progpolymsci.2014.10.001 (2015).

them with electrodes. Many of these challenges are better solved with polymer nanofibers produced by template-based or spinning methods.

\subsection{Templates}

The formation of elongated nanostructures can be assisted by templates following two conceptually different approaches. On one side, templates have been largely employed, which are made of soft matter, namely surfactants, block copolymers, liquid crystals, micelles, biomolecules, or even cells and bacteria. These have been called soft templates or pseudo-templates, and used to prime or direct the formation of polymer nanofibers by a variety of methods. On the other side, hard membranes, most of times made of polycarbonate or alumina, have been widely used as well. These membranes, which are commercially available, present a high number of nanopores (with surface density as high as $10^{11}$ pores $/ \mathrm{cm}^{2}$ ). In many studies, such pores have been infiltrated by organic molecules and precursors, thus assisting the formation of polymer nanofibers or nanotubes.

We recall in the following a few examples of soft templates developed for polymer nanofibers or nanotubes in the last decade. Nematic, cholesteric, and smectic A liquid crystal fields have been used to direct the polymerization of various compounds, such as polybithiophene, polyacetylene, polypyrrole (PPY), PEDOT and poly[2,5-di(2-furyl)toluene] [44, 64-66, 150-152] (Fig. 11). PPY nanorods have been fabricated by electropolymerization within diblock copolymer templates as well [153], and the co-assembly of triblock peptide copolymers with an amino acid-substituted polythiophene derivative through ionic interaction has been developed to obtain nanotapes [154]. PEDOT nanorods and tubes have been also realized by chemical oxidation polymerization, exploiting water-in-oil microemulsions [155]. PAN nanofibers have been produced through cylindrical micelles, exploiting amphiphilic dopants [156] or by seeding polymerization reactions with small quantities of elongated objects of different nature (peptide fibrils, other fibers, inorganic fibers etc.) [157]. Also other nanofibers, including electrospun nanofibers as those described in the next Section, can be used as template, to grow PEDOT, PPY and other conjugated materials [112, 158-165]. In this way, conductive polymers can be synthetized directly on previously realized 
Published in Progress in Polymer Science 43, 48-95, doi: 10.1016/j.progpolymsci.2014.10.001 (2015).

nanofibers, for instance through growth from precursors in vapor phase, thus forming coatings of core-shell nanostructures [166, 167], or through in-situ polymerization in solution, as recently demonstrated for PAN nanofibers on carbon nanotube templates [168]. Finally, biological molecules and supramolecular architectures including DNA, polypeptides, and amyloid fibers have been used as templates for various species of conducting and light-emitting polymer nanofibers [169-174]. In general, the concept at the base of techniques based on soft templates is using interfacial or other template-polymer intermolecular interactions in order to address the formation of nanofibers. In this way, the ultimately formed nanostructures are somehow reminiscent of the template geometry or architecture. In the most powerful methods, the template has been finely controlled through solution chemistry, or through temperature, electric or magnetic fields, which has enormously increased the possibilities of researchers in terms of finally achievable polymer nanostructures. Interesting perspectives have been also opened by using inorganic semiconductor nanocrystals as templates for polymerizing nanofibers, thus realizing bottom-up nanocomposite materials, as recently demonstrated by polythiophenes and CdTe quantum dot surfaces [175]. Indeed, small organic linkers at the surface of nanocrystals, as well as linkers which may become part of the backbone of the polymerizing conducting macromolecules, are particularly promising in order to create very effective organic-inorganic interfaces, with intriguing possibilities for realizing improved photonic and electronic junctions and devices.

In hard template methods $[176,177]$ the nanopores of membranes can be exploited to produce active nanofibers by a variety of physico-chemical mechanisms. These include, for instance, polymerization processes performed in the confined volume defined by each nano-cavity (if membranes are infiltrated by monomer solutions or vapors), or molding processes on polymers driven above their glass-transition temperature (if membranes are wetted by melts). Electrochemical polymerization of monomers is frequently used to grow nanostructures in the membranes [42, 178]. In all cases, the very high surface-to-volume ratio of nanopores makes interfacial effects highly influential on the formation of the resulting polymer nanofibers. This is especially important, since 
Published in Progress in Polymer Science 43, 48-95, doi: 10.1016/j.progpolymsci.2014.10.001 (2015).

the interactions between the polymer molecules and the walls of pores can be somehow controlled and exploited to obtain empty nanotubes or core-shell nanofibers. Even Y-shaped nanostructures have been demonstrated, using branched nanopores [58]. Finally, following synthesis, nanostructures have been separated from the membrane by dissolving the latter with proper etchant compounds, and utilized for realizing devices. Consequently, these technologies have been proven to be quite versatile, and they have allowed a wide range of polymer nanofibers to be obtained and handled. In the last decade, hard template-based nanofabrication has been applied to realize nanofibers or nanotubes by PPV [23-25], MEH-PPV [8], PVK [95, 179], poly[(9,9dioctylfluorenyl-2,7-diyl)-co-(bithiophene)] (F8T2) [52], PFO [53-59], PPY [180, 181] PEDOT [182-184] polythiophenes $[42,185]$, and other polymers, copolymers, and nanocomposites [186188]. In this framework, coupling conjugated polymers with metals ( $\mathrm{Ni}, \mathrm{Au}$, etc.) or inorganic semiconductors in hybrid nanowires is an especially relevant field of research [24, 189, 190], because these nanostructures can directly constitute contactable nanoelectronic devices. Overall, specific advantages of hard templates include the high monodispersity in size of the resulting fibers, which is related to the uniformity of the original pores, the extremely small achievable fiber diameter (a few $\mathrm{nm}$ ), and the possibility to promptly fabricate fibers which are intrinsically aligned along the direction of the pore length. These features can be quite important for some device platforms, in order to easily contact parallel nanofibers with metal electrodes or to achieve polarized light from self-aligned nanostructures. The main limitation of these technologies is in the ultimate length of the produce fibers, which is of course set by the thickness of the template membrane $(\sim$ tens of $\mu \mathrm{m})$. In order to realize longer nanofibers, up to scales of length making these materials interesting for realizing smart textiles, one needs to consider spinning technologies.

\subsection{Spinning technologies}

Today, the electrostatic spinning or electrospinning is by far the most widely used technology for the production of polymer nanofibers $[2,3,73,74,77,191-198]$. In the last decade, this method has 
Published in Progress in Polymer Science 43, 48-95, doi: 10.1016/j.progpolymsci.2014.10.001 (2015).

been largely developed in order to realize active, conductive or luminescent fibers. Electrospinning is carried out by electrified jets of polymer solutions with sufficient molecular entanglements and viscoelastic properties. The process is operationally straightforward, although it is made complex by the high number of involved experimental parameters which are related to the used polymer solution, to the applied flow and electric field conditions, and to the processing atmosphere. Briefly, the polymer solution is prepared, placed in a syringe, injected through a spinneret (usually a metallic needle) at a controlled flux, and extruded by a high applied electric field (of the order of $10^{4} \mathrm{~V} / \mathrm{m}$ ). The field is provided by a voltage bias, delivered between the spinneret and a collecting surface by means of a generator. This leads to the formation of an electrified fluid jet, which rapidly accelerates toward the collector with the possible onset of various (whipping, bending, axisymmetric, etc.) instability regimes. The solvent evaporates meanwhile [199], which allows solid nanofibers to be finally deposited on the collecting surface. An in-depth understanding of the jet dynamics and of the molecular reconfiguration in the jet is particularly important in order to predict the resulting optoelectronic properties of electrospun polymer nanofibers [200-203]. Indeed, in the jet polymer molecules have been found to undergo a dramatic stretching along the solution elongation direction. Consequently, they can align along the longitudinal axis of the resulting nanofibers [13, 91, 204], which may determine improved charge-carrier mobility and emission of polarized light. In addition, eventual phase separation phenomena occurring in the jet have been found to affect the internal nanostructure of the formed fibers, their porosity, and their surface roughness, all features which in turn influence the ultimate optical and transport properties. Phase separation phenomena have been observed to be especially relevant when spinning blends of materials with different polarity or solubility properties. Spectacular examples have been reported with copolymers $[205,206]$ or with blends of polymers with fluorescent oligomers which spontaneously form core-sheath nanofibers upon electrospinning [207]. Conjugated polymer nanofibers demonstrated in recent years have been made of PPV [26-28] derivatives such as MEHPPV [9-19], fluorene-based materials such as PFO [9, 60], poly[(9,9-dioctylfluorenyl-2,7-diyl)-co- 
Published in Progress in Polymer Science 43, 48-95, doi: 10.1016/j.progpolymsci.2014.10.001 (2015).

(1,4-benzo- $\{2,1$ ',3 $\}$-thiadiazole)] (F8BT) [14, 61], conductive systems including PAN [208], various polythiophenes [9, 12, 45-48, 209] and many others as summarized in Table 1.

One of the reasons at the base of the success of electrospinning has been its extreme experimental simplicity, which makes the fabrication of good amounts of polymer nanofibers accessible to lowcost laboratories and to researchers with basic solution chemistry and processing know-how. The versatility of this technology is another point of strength, which has given rise to a number of derived approaches. For instance, in the so-called near-field electrospinning [11, 49, 208, 210-219] the distance between the spinneret and the collector has been reduced (from many $\mathrm{cm}$ or tens of $\mathrm{cm}$ as in classical electrospinning), down to the mm-scale, or even to $0.5 \mathrm{~mm}$. This has greatly improved the capability of positioning individual fibers with high precision on pre-patterned substrates. Moreover, increasing the local electric field near the spinneret, under proper process conditions the near-field methods could lead to an enhanced orientation of permanent molecular dipoles along the fiber length. To date, the method has been developed by both piezoelectric [215] and conjugated conductive [49, 208] and light-emitting [11] polymers.

Another technique attracting increasing interest is coaxial electrospinning, in which two or more concentric apertures are used to produce jets and consequently to realize core-sheath polymer nanofibers [12, 45, 46, 220-222]. Coaxial or colloid-based [223] electrospinning methods also allow the internal flows in the jet to be somehow assisted by the viscoelastic properties of the outer solution. This has been useful for a number of applications, particularly for producing active coreshell nanofibers in which the inner region is made of or decorated by organic molecules with low entanglement, which cannot be spun alone, such as fluorescent oligomers or dyes [224]. Vice versa, other reports have shown how fluorescent polymers, such as PPV or MEH-PPV, can be used for the shell of electrospun fibers having a polyvinylpyrrolidone, PVA, or polystyrene core [19, 27]. Importantly, external sheaths can also serve as protecting coating, at least partially screening the active core against ambient humidity and oxygen, or as electrically-insulating layer, if made of 
Published in Progress in Polymer Science 43, 48-95, doi: 10.1016/j.progpolymsci.2014.10.001 (2015).

materials unable to conduct charge-carriers. Both these features are critically important to realize stable light-emitting or conductive organic nanofibers.

An increasing number of spinning technologies have been developed, which do not make use of applied external voltage bias. For examples, liquids have been manipulated with high precision, and the formation of local jets primed, by pyroelectric forces generated by temperature gradients across lithium niobate [225, 226]. This approach has been demonstrated to work by free droplets sitting on substrates, without the need for electrodes and needles, thus overcoming in principle some complications of electrospinning (particularly the clogging of needles at high polymer solution concentration). In dry spinning, no external bias is applied and viscous solutions are simply extruded from holes by means of pistons. The same solutions have been then stretched with various jet draw ratios, thus allowing micro-fibers to be achieved. Recently, dry spinning has been developed to produce light-emitting fibers made of sulfinyl-based PPV (Fig. 12) [29]. To this aim, suitable soluble precursors have been synthetized, polymerized (Fig. 12a) and then dry-spun from a viscous, $45 \%$ wt polymer solution with chloroform. The resulting fibers exhibit a diameter below $60 \mu \mathrm{m}$, become insoluble following an additional thermal treatment at $150{ }^{\circ} \mathrm{C}$, and emit bright light under UV excitation (Fig. 12b).

Scalable processes have been recently proposed, in which fiber formation is favored by high velocity expanding gas jets [227]. Melt-spinning methods also deserve to be mentioned in this framework, particularly for their good throughput and solvent-free operation. In these techniques, polymers are brough to high temperature in order to overcome their glass transition threshold and lower their viscosity, and the process can be eventually assisted by electrostatic fields to prime spinning [228-231]. Other promising candidates for massive and solvent-free production of polymer fibers include multicomponent melt spinning as that developed at Toray Industries, Inc. [232], and so-called melt-blowing methods, in which the extrusion of fibers is achieved by delivering melts through a die and assisting the processes by the aerodynamic drag of a hot air jet [233]. The 
Published in Progress in Polymer Science 43, 48-95, doi: 10.1016/j.progpolymsci.2014.10.001 (2015).

mechanisms of fiber formation during melt-blowing have been recently investigated [234, 235], and the process has been applied to the realization of multilayered filament composites [236].

\subsection{Drawing and nanopatterning}

Being controlled top-down, drawing and nanolithographic technologies have offered the highest degree of precision in positioning individual nanofibers and in interfacing the nanostructures with device platforms, electrodes, etc. In order to draw individual nanofibers or arrays of aligned nanofibers, specific serial lithographic and fabrication methods have been developed. These generally consist either in scanning a beam or a probe tip on a surface (thus driving either a polymerization reaction or the deposition of molecules defining the nanofibers). As alternative, pulling a viscous fluid body allows one to strongly reduce its diameter up to obtaining a very thin filament.

In the first class of methods, both electron-beam lithography and dip-pen nanolithography have been used to locally cure polymers with nm-resolution, thus obtaining nanofibers whose chemical composition, density, or optical and electrical properties are different from those of the unexposed material. A recently developed approach in this respect is the electron-beam exposure of polyacrylamide nanofibers, which are initially nonluminescent and become luminescent once activated by the irradiation [85]. The resulting nanomaterial has been found to be quite stable against photobleaching, and to exhibit an increase of about 0.1 in its refractive index. Also electrospun nanofibers doped with metallo-organic precursors and then exposed to electron-beams have been found to become luminescent, due to the in-situ generation of light-emitting quantum dots which is activated by the irradiation [237]. In the framework of scanning probe methods, thermochemical nanopatterning [30] has been also demonstrated, which consists in scanning a heated tip or wire on a polymer precursor layer. Upon passing on the film, the wire locally heats and cross-links the polymer. This method has been applied to fabricate crossed, light-emitting nanofibers of PPV, with lateral resolution at the scale of a few tens of nm (Fig. 13). 
Published in Progress in Polymer Science 43, 48-95, doi: 10.1016/j.progpolymsci.2014.10.001 (2015).

Polymer nanofibers based on conjugated polymers could be also produced by nanofluidics, exploiting the spontaneous or pressure-driven penetration of polymer solutions into nanochannels. To this aim, the template capillaries have been fabricated by a substrate and an elastomeric mold placed in conformal contact onto the substrate, as in soft lithography. Some nanofluidic methods have been optimized by using mold materials which are able to resist against the eventual swelling by the organic solvents normally employed to make solutions with conjugated polymers [21]. Other techniques make use of prefabricated electrode templates to polymerize electrochemically some fluidic precursors in nano-channels, allowing arrays of heterogeneous conjugated polymer nanowires to be realized with high precision [238]. Microfluidic synthesis can be used to produce light-emitting nanofibers as well, particularly by so-called continuous flow lithographies which are based on one- [239] or two-photon [240] curing of organic materials flowing in micro-channels. In this way, the process is continuous, and highly controlled in its conditions. In particular, by using suitable photopolymerization spots with negative polymeric resists hosting fluorescent dopants, self-standing helical light-emitting nanofibers have been demonstrated.

Drawing methods have been developed in many variants, using either melts or polymer solutions, in order to produce light-emitting nanofibers [83-85, 241, 242]. Finally, one should recall that different lithographic technologies can be combined in many ways in order to produce or pattern active polymer nanofibers $[14,243]$. Some of the resulting hybrid methods constitute elegant examples of merging of top-down and bottom-up lithographies [197]. For instance, PEDOT:poly(styrenesulfonate) (PSS) nanowires have been realized through etching masks, in turn produced by block copolymer lithography with poly(styrene- $b$-dimethylsiloxane) [243]. Block copolymer lithography is particularly advantageous in this respect, since it allows large areas to be patterned in parallel, thus overcoming many drawbacks of high cost and low throughput of serial writing methods. 
Published in Progress in Polymer Science 43, 48-95, doi: 10.1016/j.progpolymsci.2014.10.001 (2015).

\section{Optoelectronics}

Realizing nanofibers by conjugated polymers or other organic materials have led to the observation of enhanced photonic properties, which in turn have contributed to make these nanostructures extremely appealing for optoelectronic devices [241, 244-246]. In fact, the improved properties have been often determined by a more ordered internal structure, enhanced crystallization, or higher degree of mutual alignment of molecules [247], all effects primed in nanofibers during fabrication and synthesis steps. This is, for instance, the case of PFO, for which an enhanced content of the $\beta$ phase [56] has been reported in nanofibers realized by hard templates (Sec. 3.2). Since the $\beta$-phase is usually related to a highly extended conjugation length, and to fundamental optical properties including higher luminescence yield and favored stimulated emission, it is clear that PFO nanowires exhibiting higher content of this crystalline phase are better candidates (compared, for example, to thin films) as active media for various devices including nanoscale OLEDs and solid-state organic lasers. Another important characteristic is the internal orientation of macromolecules along the longitudinal axis of the nanofibers, which has been found to induce significant polarization of the emitted light as mentioned in Sec. $2.2[13,89,91,204]$. These features have been found to be quite general and to some extent independent from the particular nanofabrication method. For example, in electrospinning molecular alignment has been investigated by a number of approaches including polarized Fourier transform infrared spectroscopy, polarized Raman scattering, polarized photoluminescence, and X-ray diffraction, and associated with the large stretching induced in the electrified jets due to the applied electric field [13, 91, 204]. In completely different experiments, in which aligned polymer filaments have been produced following incorporation of MEH-PPV into oriented mesoporous silica, Molekamp et al. have observed highly polarized PL emission [89], and so on. 
Published in Progress in Polymer Science 43, 48-95, doi: 10.1016/j.progpolymsci.2014.10.001 (2015).

Furthermore, various evidences have been reported, that polymer nanofibers may emit brighter light compared to films. In particular, PPV nanotubes [23] and F8BT nanofibers [248] show a quantum yield which is significantly higher than those of the corresponding thin films (39-46\% vs. 19-22\% and $40 \%$ vs. $27 \%$, respectively). Furthermore, the emission efficiency in fibers is strongly dependent on their particular microstructure, which can be thermally-induced as investigated in detail in the case of electrospun PFO [60]. Other interesting properties have been highlighted by a photophysical study of P3HT-block-poly(3-methanolthiophene) (P3HT-b-P3MT) and P3HT-blockpoly-(3-hexylthioacetate thiophene) (P3HT- $b$-P3ST) nanofibers [249]. Indeed, the resulting photophysics has been found to depend significantly on the specific functionalization and crosslinking, affecting strain and aggregation at molecular scale.

Finally, miniaturization and greatly increased surface-to-volume ratios are other obvious and advantageous consequences of using polymer nanofibers as active material for organic optoelectronics, opening new possibilities for the realization of low-cost nanoscale light sources, and for the development of improved optical sensors [83, 250]. On the other hand, the integration of large-area optoelectronics based on polymer nanofibers with smart textiles may be another, very interesting route for next-generation hybrid technologies.

\subsection{Light emitting devices and lasers}

For all the reasons presented above, at least three classes of light-emitting devices might benefit from using polymer nanofibers as active medium: OLEDs and organic light-emitting transistors (OLETs), which are electrically-pumped, and miniaturized lasers, which have been opticallypumped hitherto. For devices relying on electric injection and on radiative recombination of chargecarriers, such as OLEDs and OLETs, properly coupling polymer nanofibers with electrodes is of course one of the most critical issues. For instance, OLED architectures are typically realized by sandwiching the light-emitting and charge-transporting layers between the anode and the cathode. The two electrodes serve for injecting holes and electrons, respectively, and the recombination of charges with opposite signs then leads to radiative emission. For an efficient injection, the work 
Published in Progress in Polymer Science 43, 48-95, doi: 10.1016/j.progpolymsci.2014.10.001 (2015).

functions of involved metals have to match as closely as possible the HOMO and LUMO levels, respectively, of sandwiched organic materials. Several recent reviews exist on OLEDs and on the solution-processing of the related active materials [251-253], to which interested readers are referred for more details. These technologies are now very well established, and widely commercialized, for planar systems such as thin-film OLEDs. However, employing non-planar active nanostructures such as nanofibers requires a further development in terms of layer deposition and device configuration, in order to warrant an effective coupling with electrodes and hence electrical injection. In this framework, an elegant approach has been proposed by Vohra and coworkers [61], who have electrospun light-emitting F8BT-based nanofibers with a significant content of PEO (35\%). These nanofibers have been deposited on plasma-treated indium tin oxide/PEDOT:PSS, annealed at a temperature of $150^{\circ} \mathrm{C}$ which is higher than the melting temperature of PEO $\left(65^{\circ} \mathrm{C}\right)$, and then coated with spin-cast PVK and with a $\mathrm{Ca} / \mathrm{Al}$ cathode (Fig. 14). The annealing step is the most important during device preparation, because at $150^{\circ} \mathrm{C}$ the two polymer components have been found to separate. On one side, this leads to the formation of a continuous, electrically-insulating PEO layer, which serves as spacer between PEDOT:PSS and PVK thus blocking undesired current contributions not passing through the nanowires. On the other side, annealing renders F8BT nanofibers thinner than as-spun ones (down to a thickness of $150 \mathrm{~nm}$, making them more suitable to transport and radiative recombination of charge-carriers), and largely insoluble in toluene (used to spin the PVK layer on top of the fibers). Overall, this approach has shown a large number of advantages for the production of OLEDs based on light-emitting nanofibers. The resulting devices have exhibited electroluminescence for voltages above $6 \mathrm{~V}$, and luminances of $2300 \mathrm{~cd} / \mathrm{m}^{2}$ which are much higher than in samples with untreated nanofibers (36 $\left.\mathrm{cd} / \mathrm{m}^{2}\right)$.

In another, pioneering work by the Redmond group, PFO nanowires have been used as active material following their deposition on PEDOT:PSS in a glove box and Al evaporation on top, similarly to standard OLEDs [56]. This structure, whose working conditions are likely remarkably 
Published in Progress in Polymer Science 43, 48-95, doi: 10.1016/j.progpolymsci.2014.10.001 (2015).

affected by the overall surface density and networking of the deposited mat of nanowires, as well as by the enhanced content of the $\beta$-phase in the PFO nanostructures, has been reported to exhibit a threshold of $3.4 \mathrm{~V}$ for electroluminescence (Fig. 15). The same nanowires have been also used within light-emitting electrochemical cells [56]. Of course, it is likely that in the near future nanofiber-based devices based on a layer-by-layer structure will further benefit from exploiting extra layers for efficient hole- and electron injection and transport, as in conventional OLEDs. A third route has used instead a planar geometry, in which nanofibers are not sandwiched between anode and cathode, but instead bridge the electrodes similarly to FETs. This has been developed by Moran-Mirabal et al. and again has involved electrospun PEO nanofibers (this time doped by a redemitting, ruthenium-based complex) [69]. The fibers, with diameter between $150 \mathrm{~nm}$ and micrometers depending on the electrospinning parameters and PEO amount in the spun solution, have been directly deposited onto micropatterned gold interdigitated electrodes, realized on an insulating silica layer. For an inter-electrode spacing of $500 \mathrm{~nm}$, electroluminescence has been observed from fiber regions of size below $240 \times 325 \mathrm{~nm}^{2}$, for applied voltages above about $3 \mathrm{~V}$ (Fig. 16a). This geometry is suitable also for achieving light-emission from multiple inter-electrode gaps as shown in Fig. 16b-d, and for implementing OLETs [254] based on electroluminescent polymer nanofibers.

A fourth possibility has been opened by the implementation of light-emitting devices directly at single-nanofiber level, injecting charges at the core and at the surface of each individual fiber. This has been demonstrated by co-electrospinning methods [255], fabricating fiber-shaped heterostructures which have a liquid metal core, a ionic transition-metal complex polymer shell, and an external indium tin oxide coating. The measured thresholds for light emission from these radial OLED architectures have been of 4-5 V, and luminances have been of $23 \mathrm{~cd} / \mathrm{m}^{2}$. These fibers are clearly a significant advancement in view of the integration of electroluminescent devices with smart textiles. 
Published in Progress in Polymer Science 43, 48-95, doi: 10.1016/j.progpolymsci.2014.10.001 (2015).

A still different approach has been developed in the Nanofiber Light Improvement Technology (NLITe ${ }^{\mathrm{TM}}$ ) by Davis and coworkers at RTI International, allowing issues related to nanofiber/electrode coupling to be completely overcome [256]. In this case, highly reflective surfaces (with reflectance of $95 \%$ ) have been produced by nanofibers, and the photoluminescence of light-emitting nanofibers doped with quantum dots has been exploited instead of electroluminescence. Some examples of the pristine solutions and of the light-emitting nanofibercovered surfaces are shown in Fig. 17. For instance, large-area nanofiber mats have been mounted on lamp sockets and excited by means of standard blue LEDs. This has ultimately led to the realization of bright white light sources by nanofibers. So far, this technology has been unique in targeting illuminating engineering and design by means of active polymer nanofibers. Advantages are numerous, including low energy consumption and enjoyable illumination conditions due to good color rendering and light diffusion from the nanofiber surfaces. At the time of the preparation of this review, detailed information about NLITe ${ }^{\mathrm{TM}}$, including application notes and publications, has been made available at the RTI International website [256].

The investigation of the amplified spontaneous emission and lasing properties of polymer nanofibers is still at the beginning stage. It is clear that the peculiar supramolecular organization found in many nanofiber species directly affects optical transitions, and as such it is also expected to influence the stimulated emission cross-section of conjugated materials. However, this fundamental aspect is still unexplored, whereas pioneering works on lasing polymer nanofibers have been mainly focused on issues related to the cavity geometry [54, 68, 257]. In 2007, O'Carroll, Lieberwirth and Redmond have reported on PFO nanowires which show a clear Fabry-Pérot mode structure in their emission [54]. Following excitation with $0.7 \mathrm{~ns}$ pulses at $355 \mathrm{~nm}$ above a pumping threshold of 1.7-3.0 $\mathrm{mJ} \mathrm{cm}^{-2}$, these nanowires exhibit line-narrowing which is in agreement with the occurrence of lasing action. A prevalently single-mode emission has been observed. Furthermore, the emission collected from the tip (but not from the body) of the nanowires has shown a spectral modulation which is ascribable to resonances associated with an axial Fabry-Pérot microcavity. In 
Published in Progress in Polymer Science 43, 48-95, doi: 10.1016/j.progpolymsci.2014.10.001 (2015).

these systems, the microcavity is associated with the nanowire body, and reflections of internally guided light can occur at the end faces of the nanostructures (Fig. 18). This has been modeled by means of the following formula:

$$
\Delta \lambda_{\mathrm{c}}=\left(\frac{\lambda_{\mathrm{c}}^{2}}{2 L}\right)\left(n-\left.\lambda_{c} \frac{\mathrm{d} n}{\mathrm{~d} \lambda}\right|_{\lambda_{c}}\right)^{-1}
$$

where $\Delta \lambda_{\mathrm{c}}$ is the spacing between modes in the spectrum, $\lambda_{\mathrm{c}}$ is the wavelength of the central peak of the modulated photoluminescence, $L$ is the wire length, and $\mathrm{d} n / \mathrm{d} \lambda$ indicates the wavelength dispersion of the refractive index.

In 2009, Camposeo and coworkers have found a largely analogous behavior in electrospun lightemitting nanofibers embedding optically gaining organic dyes such as rhodamine (Fig. 19a,b) [68]. These nanofibers have been excited by $0.6 \mathrm{~ns}$ pulses at $532 \mathrm{~nm}$, exhibiting spectral line-narrowing above a threshold of $60 \mu \mathrm{J} \mathrm{cm}^{-2}$. Complex spectra, with a large number of very narrow peaks, have been observed in correspondence of nanofibers with a significant dispersion of their sizes (Fig. 19c). Although further investigation is likely needed in this respect, the occurrence of random lasing has been initially ruled out due to the stable number and spectral positions of these narrow modes. On the contrary, single-fiber spectra are consistent with Fabry-Pérot resonances as those predicted by Eq. (1).

In 2011, Das and coworkers have investigated electrospun nanofibers made of polystyrene embedding the dye 4-dicyanomethylene-2-methyl-6-p-dimethylaminostyryl-4H-pyran (DCM) [257]. These nanofibers have been excited by $0.5 \mathrm{~ns}$ pulses at $532 \mathrm{~nm}$, and found to exhibit a threshold of $1 \mu \mathrm{J}$ or $200 \mathrm{~nJ}$ when only one or both the edges of the fibers are cleaved, respectively (Fig. 20). This finding supports the occurrence of a significant axial feedback along the length of the fiber, as associated to reflectivity from end faces. However since the emission spectra have been collected from the fiber body, this work has also suggested the onset of complex, three-dimensional resonance schemes. Overall, it is likely that the different fabrication technologies developed to 
Published in Progress in Polymer Science 43, 48-95, doi: 10.1016/j.progpolymsci.2014.10.001 (2015).

produce active polymer nanofibers, as well as post-synthesis processing methods, can be optimized in order to control the local morphology and ultimately the reflectivity of the end facets and of the body surface of these nanostructures.

One should also point out that, by virtue of their cross-section which is frequently and intrinsically circular, polymer fibers can also generate whispering gallery modes, in which resonances are given by light travelling around a concave surface. These modes show numerous advantages due to their high quality factors and small mode volumes, which are in turn related to low thresholds for lasing. A beautiful demonstration of miniaturized lasers based on whispering gallery modes has been recently provided by drawn polymer microfibers doped with rhodamine (Fig. 21) [258]. Upon excitation with $1 \mathrm{~ns}$ pulses at $532 \mathrm{~nm}$, these microfibers have shown a threshold of $400 \mathrm{~nJ}$.

Finally, a distributed feedback laser, in which a grating has been imprinted on the top surface of an electrospun light-emitting nanofiber, has been demonstrated in 2014 [259]. This device has been reported to show emission peaked at $562 \mathrm{~nm}$, and a lasing threshold significantly reduced compared to thin films based on the same active material.

These studies, besides opening significant perspectives for the realization of optical circuits and other integrated systems based on lasing nanofibers, are going to stimulate next fundamental work in order to better assess microcavity effects in these organic nanostructures, and widen the set of photonic effects (i.e. random lasing, polaritons, etc.) observable in them.

\subsection{Photodetectors and photovoltaics}

Nowadays, organic optoelectronics has become a strategic field not only for the realization of novel light-sources and displays, but also for the exploitation of renewable energy. Indeed, conjugated polymers and many low-molar-mass molecules show interesting light-absorption properties (with absorption coefficient above $10^{5} \mathrm{~cm}^{-1}$ ), making them interesting candidates for building photodetectors and other devices including organic solar cells [260-262]. To collect photogenerated charges, however, not only absorption spectra have to be intense and to match the frequency windows of the radiation in which one is interested (specific laser light, light from lamps, sunlight, 
Published in Progress in Polymer Science 43, 48-95, doi: 10.1016/j.progpolymsci.2014.10.001 (2015).

etc.), but charge pairs have to be dissociated and the transport of carriers should be as efficient as possible, thus allowing the separated carriers to reach electrodes coupling the device with an external circuit. These issues are rendered even more complex by the eventual interplay existing between absorption and conduction properties in organics. It is clear that polymer nanofibers can be promising candidates in this respect, showing absorption features resembling those of the pristine materials as well as generally improved charge-carrier transport. Consequently, in the last decade the application of active polymer nanofibers to photovoltaics has stimulated an increasing curiosity of researchers. However, photovoltaic devices based on nanofibers are evidently affected by all those effects of organics/electrode coupling, and by the related complications, mentioned in the previous Section about OLEDs. For this reason, special care is necessary in all the synthesis and fabrication processes leading to this class of devices, particularly in the steps affecting the transport performances of nanofibers both in the polymer material and at its interfaces with electrodes. Concerning photodetectors, different approaches have been reported in the literature. We will review them in chronological order. Hybrid nanostructures made of conjugated polymers and either carbon or metals or semiconductors have been studied most widely in this framework, due to their better conductivity properties compared to barely organic nanostructures. In 2005, photoconductivity has been studied in single-bilayer nanotubes of PPV and carbonized PPV [263]. In that work, the PPV nanotubes have been produced by chemical vapor deposition polymerization within the pores of a membrane, and partially carbonized. The nanotubes have been contacted following the deposition of a drop of solution onto photolithographically-made gold microelectrodes, exhibiting a responsivity of 0.012-0.016 $\mathrm{mA} \mathrm{W}^{-1}$, and external quantum efficiencies of 4-6\% (under illumination with a Xe lamp with an irradiance of $1.5 \mathrm{~W} \mathrm{~cm}^{-2}$ ). In particular, the responsivity, in units of $\mathrm{A} \mathrm{W}^{-1}$, is a relevant parameter to assess the device performances, since it indicates the amount of photogenerated current per unit incident power of light. In 2006, single F8T2 nanowires, realized by wetting porous anodized alumina membranes as described in Sec. 3.2, have been firstly contacted by drop deposition onto prepatterned electrodes on 
Published in Progress in Polymer Science 43, 48-95, doi: 10.1016/j.progpolymsci.2014.10.001 (2015).

oxidized silicon chips [52]. This corresponds to a bottom-contact geometry, similar to that reported in the previous work, simple to realize and useful to characterize the (injection-limited) transport in the nanowires. Then, the same nanostructures have been used in a top-contact device, which has exhibited a responsivity of about $0.4 \mathrm{~mA} \mathrm{~W}^{-1}$ and an external quantum efficiency around approximately $0.1 \%$ under monochromatic illumination (at a laser wavelength of $405 \mathrm{~nm}$ and with an irradiance of $40 \mathrm{~W} \mathrm{~cm}^{-2}$ ). Interestingly, the responsivity measured for single F8T2 nanowires is comparable to those in other, previous reports on inorganic nanowires. A top-contact device architecture has been also used by Guo et al. in 2008, to investigate the photoconduction properties of hybrid, organic/inorganic $p-n$ junctions supported in nanowires made of PPY and CdS (Fig. 22) [190]. These junctions show a rectifying behavior which is light-dependent. In 2010, bulkheterojunction PPV-CdSe fibers have been realized, showing a spectral response of their photocurrent peaked at $500 \mathrm{~nm}$, together with a switch ratio close to five orders of magnitude under conditions of illumination with white light at $100 \mathrm{~mW} \mathrm{~cm}^{-2}$ and a bias voltage of $10 \mathrm{~V} \mathrm{[264].}$ Analogous fibers have been produced with PPV and $\mathrm{Fe}_{3} \mathrm{O}_{4}$ nanoparticles, allowing the orientation of nanostructures to be controlled by magnetic fields during electrospinning [265]. Finally, in 2012 Imahori and coworkers have demonstrated bottom-contact devices based on nanorods with two gold terminations and an intermediate segment of P3HT and fullerene, which show a decrease of their resistivity by more than one order of magnitude following illumination with white light [266]. In 2012, the photoconductivity of polyacrylonitrile/PAN core-shell nanofibers has also been investigated, exhibiting a fast response and a resistivity switch ratio exceeding four orders of magnitude [162].

In phototransistors using polymer nanofibers, the device geometry is enhanced by a third electrode acting as gate and allowing the characteristics to be tuned. For instance, increasing gate voltage has been reported to lead to a decrease of the photoluminescence from MEH-PPV nanofibers [267]. These devices have shown that the conductivity of nanofibers can be controlled through external illumination, with relaxation times of the photo-induced current of the order of tens of seconds 
Published in Progress in Polymer Science 43, 48-95, doi: 10.1016/j.progpolymsci.2014.10.001 (2015).

[268]. Here, the photoresponsivity is of $100 \mathrm{~mA} / \mathrm{W}$ for a MEH-PPV fiber with a diameter of 500 $\mathrm{nm}$. However, this class of components is still poorly explored and will deserve further investigation in the near future.

For photovoltaic devices, another, important figure of merit is the so-called fill factor, $F F=V_{M} I_{M} /$ $V_{O C} I_{S C}$, where $V_{M}$ and $I_{M}$ are the voltage and the current achieved at maximum power output, respectively, $V_{O C}$ is the open-circuit generated bias, and $I_{S C}$ is the short-circuit current. $F F$ so indicates the power conversion efficiency of the cell. For instance, MEH-PPV nanotubules of 200 nm and 100 nm show $F F=59 \%$ and $F F=67 \%$, respectively, which are significantly higher that values measured by MEH-PPV thin films (20-30\%) [8]. The fill factor is especially relevant for assessing the performances of solar cells, which have to collect sunlight in order to generate electricity. The typical device architecture has been based on sandwiching the active organics between indium tin oxide/PEDOT:PSS and a capping cathode, as in light-emitting diodes. Also in this field, active polymer nanofibers begin to be widely exploited, as building block which serves either as one of the two parts of bulk heterojunctions (for instance, using fibers as dopant in nanocomposite layers) or as carrier of junctions formed within the same nanostructures. In the resulting devices, power conversion efficiencies are of the order of a few percent. For instance, a study by the Jenekhe group has been focused on the photovoltaic properties of self-assembled nanowires of regioregular poly(3-pentylthiophene) (Fig. 23) [269]. Bulk heterojunction devices have been formed by layers incorporating the nanofibers (electron-donor) in $[6,6]-$ phenyl- $\mathrm{C}_{61}$ butyric acid methyl ester (PCBM) or [6,6]-phenyl $\mathrm{C}_{71}$-butyric acid methyl ester (acceptor). Under $100 \mathrm{~mW} / \mathrm{cm}^{2}$ AM1.5 sunlight illumination, these solar cells have shown a short-circuit current density up to $9.8 \mathrm{~mA} \mathrm{~cm}^{-2}, V_{O C}=0.54-0.56 \mathrm{~V}$, and $F F=63-65 \%$. Another report deals instead with poly(3-butylthiophene) nanofibers [270]. Furthermore, a systematic investigation varying the drying and annealing conditions has allowed the authors to observe that the internal structure of the nanocomposite layers and the density and morphology of the network of nanofibers critically affect the photovoltaic performances [271]. Indeed, dense and random webs of nanofibers leads the 
Published in Progress in Polymer Science 43, 48-95, doi: 10.1016/j.progpolymsci.2014.10.001 (2015).

acceptor component to aggregate in the small regions where fibers are not present. The nanocomposite layer so presents inter-penetrating domains of the two polymer species, and the resulting devices have shown high short-circuit current and relatively low $V_{O C}$. On the contrary, reducing the density of the nanofibers leads to lower currents and higher $V_{O C}$ values, with the presence of random bulk heterojunctions of spatially distributed acceptor and donor phases. These results have indicated that an optimal device structure can be obtained by retaining inter-penetrating domains of the two polymers, thus using highly-dense mats, but without the effect of bridging the two electrodes (hence reducing $V_{O C}$ ) which is associated with long poly(3-butylthiophene) nanofibers. Sonication can be another effective route to this aim, favoring the achievement of a homogeneous dispersion of the acceptor component nearby the surface of electron-donor nanofibers as shown in Fig. 24 [272]. The resulting solar cells have been found to exhibit a short-circuit current density up to $10.4 \mathrm{~mA} \mathrm{~cm}^{-2}, V_{O C}$ of $0.61 \mathrm{~V}$, and a fill factor up to 64-65\%. In another study, meltassisted wetting in porous membranes has allowed Wang et al. to produce arrays of core-shell nanofibers made of an external P3HT region and internal PCBM [43]. These nanostructures naturally form heterojunctions across the two polymers, thus allowing to apply in nanofibers a strategy which is largely used in organic solar cells. For instance, devices based on P3HT and PCBM have exhibited a short-circuit current density of 4.4-5.9 $\mathrm{mA} \mathrm{cm}^{-2}, V_{O C}=0.40-0.42 \mathrm{~V}$, and $F F=38-53 \%$, depending on the relative concentrations of the two polymers used to prepare the nanostructures. Annealing at $120^{\circ} \mathrm{C}$ has increased the short-circuit current density up to $8.7 \mathrm{~mA} \mathrm{~cm}^{-2}$ [43]. Good performances, improving those of bulk heterojunction devices, have been also reported by embedded electrospun poly[N-9'-hepta-decanyl-2,7-carbazole-alt-5,5-(4',7'-di-2thienyl-2',1',3'-benzothiadiazole)]-based nanofibers which promote exciton dissociation [273]. A recent study by Seidler et al. has been focused on P3HT nanofibers used in multilayer solar cells, which have been obtained through the successive deposition of the donor and the acceptor (PCBM) system, from solution based on chloroform (Fig. 25) [274]. While the crystallinity and film retention of the deposited layers can be controlled by the relative weight of di-tert-butyl peroxide 
Published in Progress in Polymer Science 43, 48-95, doi: 10.1016/j.progpolymsci.2014.10.001 (2015).

and chloroform in the solutions, the effect of adding di-tert-butyl peroxide is mainly directed to form networks of nanofibers and consequently improve hole mobility and increase the insolubility of the deposited P3HT. This in turn allows the acceptor to be subsequently cast by the same solvent. The resulting devices have been found to exhibit a short-circuit current density of 6.2-8.4 mA cm${ }^{-2}$ and $F F=40-52 \%$, depending on the annealing conditions. More in general, thermal annealing may be carried out in order to prime the formation of a network of P3HT fibrils directly in blend films, thus allowing to realize in-situ bulk heterojunctions based on conductive polymer nanofibers [275]. These cells have shown a short-circuit current density of $7.2 \mathrm{~mA} \mathrm{~cm}{ }^{-2}, V_{O C}=0.61 \mathrm{~V}$, and $F F=61 \%$. In another work with in-situ self-assembled poly(3-butylthiophene) nanofibers, cells have been found to reach short-circuit current densities of 3.4-9.0 mA cm${ }^{-2}, V_{O C}=0.57-0.61 \mathrm{~V}$, and $F F=35$ $56 \%$ [276], thus being comparable with analogous devices obtained by pre-assembling the nanofibers in solution and then dispersing them in the donor component [270]. Finally, dyesensitized solar cells based on PEDOT nanofiber counterelectrodes have been demonstrated to outperform similar devices, exploiting bulk PEDOT, in terms of ultimate conversion efficiency, and to reach current densities of $17.5 \mathrm{~mA} \mathrm{~cm}^{-2}, V_{O C}=0.72 \mathrm{~V}$, and $F F=72-73 \%$ [277]. Good performances have been also obtained by means of counterelectrodes based on electrospun conductive PAN/polylactic acid nanofibers [278].

As mentioned above, a very interesting strand of research which may open important perspectives in the near future is using active polymer nanofibers in smart textiles. Photovoltaic textiles would be especially relevant in this framework since their embedment in clothes or other objects of daily use (window shades, lens hoods, visors, parasols, furniture etc.) could enormously increase the number of surfaces available for sunlight collection and clean energy generation worldwide, and improve the related applications in personal and biomedical devices, smart phones, and domotics. For realizing photovoltaic textiles, however, issues related to nanofiber/electrode coupling become even more serious, as well as those associated with the eventual robustness of the realized nanofiber fabrics. A few recent advancements deserve to be mentioned in this respect. A pioneering work in 
Published in Progress in Polymer Science 43, 48-95, doi: 10.1016/j.progpolymsci.2014.10.001 (2015).

this framework has been carried out by the Ramakrishna group, reporting a non-woven composed of P3HT/PCBM electrospun nanofibers with short-circuit current densities of $3.2 \mathrm{nA} \mathrm{cm}^{-2}, V_{O C}=$ $0.12 \mathrm{~V}$, and $F F=22 \%$ [279]. While the conversion efficiency of these photovoltaic non-wovens is still very low $\left(<10^{-7}\right)$, the authors have proposed that various strategies can be developed in order to improve it in future, including the use of inert atmosphere during the synthesis and processing of nanofibers.

\section{Nanoelectronics}

An important finding making polymer nanofibers very interesting for nanoelectronic devices, such as FETs, has been that their charge-carrier mobilities are frequently orders of magnitude higher than those in thin films. For instance, MEH-PPV nanotubules of $200 \mathrm{~nm}$ diameter, made by hard template wetting (Sec. 3.2), have shown a hole mobility, $\mu_{\mathrm{H}}$, of $10^{-5} \mathrm{~cm}^{2} / \mathrm{V} \mathrm{s}$, which is one order of magnitude larger than in films [8]. $\mu_{\mathrm{H}}$ has been found to further increase up to $10^{-3} \mathrm{~cm}^{2} / \mathrm{V}$ s for MEH-PPV nanotubules with a diameter of $100 \mathrm{~nm}$. Similar results have been obtained in P3HT nanofibers, for which a mobility of $10^{-1} \mathrm{~cm}^{2} / \mathrm{V}$ s has been measured, which is 60 times higher than in films [50]. In particular, for these compounds the processes of fabrication of nanofibers have been often found to favor a molecular reassembly processes, enhancing the alignment of polymer backbones and the $\pi-\pi$ stacking along the longitudinal axis of the nanostructures. This effect is more and more relevant upon decreasing the diameter of the nanostructures below $100 \mathrm{~nm}$, and it has been reported for a number of different spinning and nanopatterning techniques including electrospinning, solvent-assisted molding [50, 280], and nanoimprint lithography [281, 282]. Another interesting example is given by a diketopyrrolopyrrole-benzothiadiazole-based copolymer, which has shown an ambipolar behavior with a hole and electron mobility up to $0.27 \mathrm{~cm}^{2} / \mathrm{V} \mathrm{s}$ and $0.34 \mathrm{~cm}^{2} / \mathrm{V} \mathrm{s}$, respectively, following thermal annealing of thin films [283]. These values have been increased up to $0.70 \mathrm{~cm}^{2} / \mathrm{V} \mathrm{s}$ and $1.45 \mathrm{~cm}^{2} / \mathrm{V} \mathrm{s}$, respectively, when spin-cast layers have been 
Published in Progress in Polymer Science 43, 48-95, doi: 10.1016/j.progpolymsci.2014.10.001 (2015).

replaced by parallel nano-stripes realized by lithographically-controlled wetting. Also PEDOT nanowires produced by electrochemical polymerization assisted by hard templates (Sec. 3.2) have been found to exhibit an increase of their conductivity by almost two orders of magnitude upon decreasing the diameter from $190 \mathrm{~nm}$ to $35 \mathrm{~nm}$ [183, 284]. Analogous results have been reported for nanoimprinted features of the liquid-crystalline polymer F8BT, in which backbones align parallel to the length of the features leading to an increase of the hole and electron mobility from $4-6 \times 10^{-4}$ $\mathrm{cm}^{2} / \mathrm{V} \mathrm{s}$ and $1-2 \times 10^{-4} \mathrm{~cm}^{2} / \mathrm{V}$ s to $8 \times 10^{-4}-1.2 \times 10^{-3} \mathrm{~cm}^{2} / \mathrm{V}$ s and to $2-3 \times 10^{-4} \mathrm{~cm}^{2} / \mathrm{V}$ s, respectively [62]. Of course, a lot of research has been carried out in the attempt to exploit such improved properties of conductive polymer nanofibers in FET devices and in logic gates and circuits.

\subsection{Field-effect transistors}

Various electronic components, and particularly Schottky nanodiodes [285, 286] and two-terminal chemical sensors [161, 287-289], have been realized by using conductive polymer nanofibers as active material. Four-probe devices have been presented as well, and utilized for fundamental studies of both the conductivity [290] and the magnetoresistance of polymer nanofibers and nanotubes $[129,183,291,292]$. These devices are remarkable due to their inherent simplicity, robustness, and straightforward realization. However, FETs are certainly the most interesting devices for electronics, due to the possibility to combine them in complementary metal oxide semiconductor-like logic gates and other more complex, integrated circuits. Many evidences have been reported that the performances of organic FETs critically depend not only on the used conjugated polymers, but also on the details of the device architectures, on the employed dielectric layer and on its functionalization, etc. In the following, for sake of simplicity we will review progresses in polymer nanofiber-based FETs by referring only to the active medium. Interested readers are encouraged to look for the device fabrication and processing details in the original references. 
Published in Progress in Polymer Science 43, 48-95, doi: 10.1016/j.progpolymsci.2014.10.001 (2015).

Early reports on electrospun camphorsulfonic-acid-doped PAN/PEO nanofibers have demonstrated hole mobilities of $1.4 \times 10^{-4} \mathrm{~cm}^{2} / \mathrm{V}$ s in the depletion regime [293]. Films of P3HT nanofibers obtained by polymerization in solution [294] have shown $\mu_{\mathrm{H}}=2.7 \times 10^{-3} \mathrm{~cm}^{2} / \mathrm{V} \mathrm{s}$, and current on/off ratios of $10^{3}$ [51]. P3AT nanowires produced by electrospinning or self-assembled from toluene, chloroform, or other solution casting approaches have allowed values of $\mu_{\mathrm{H}}$ of $(1-7) \times 10^{-2} \mathrm{~cm}^{2} / \mathrm{V} \mathrm{s}$ to be reached $[33,39,41,45,133,135,295,296]$. The typical characteristics, showing the dependence of the drain-source current, $I_{D}$, on the drain-source voltage, $V_{D}$, for various values of the gate voltage, $V_{G}$, are shown in Fig. 26 together with the morphology of networks of nanofibers made of P3ATs with different alkyl chain length [33]. Overall, recent studies suggest that in these compounds the impact of the alkyl chain length on the FET mobility, if any, is mainly indirect, through the influence of these chains on the molecular order at the organic/dielectric interface [297]. More recently, various unconventional approaches have been developed in order to improve considerably performances in FETs based on P3HT nanofibers. In a method, polyelectrolyte gate dielectrics based on UV-crosslinked hydrogels have been used together with electrospun P3HT fibers, allowing values of $\mu_{\mathrm{H}}$ as high as $2 \mathrm{~cm}^{2} / \mathrm{V}$ s to be measured [46]. In this and other studies, P3AT-based nanofibers have been reported to be very interesting systems for building flexible FETs, in which they offer good bending stability [209]. In another method, charge-carrier transport has been improved relying on Au-impregnated polymer nanofibers made of polyacrylonitrile and polythiopene in a core-shell architecture [166]. Also these devices have been found to reach $\mu_{\mathrm{H}}$ of 2 $\mathrm{cm}^{2} / \mathrm{V}$ s. Finally, the electrohydrodynamic lithographic deposition of organic nanowires has allowed values of field-effect mobility as high as $9.7 \mathrm{~cm}^{2} / \mathrm{V}$ s to be recorded by P3HT-based nanostructures with ion-gel dielectrics (Fig. 27) [49]. Current on/off ratios in these devices exceed $10^{5}$

Another breakthrough result reported for $p$-type nanofiber FETs has been obtained by cyclopentadithiophene-benzothiadiazole, reaching $\mu_{\mathrm{H}}$ of $3.3-5.5 \mathrm{~cm}^{2} / \mathrm{V} \mathrm{s}$, and current on/off ratios 
Published in Progress in Polymer Science 43, 48-95, doi: 10.1016/j.progpolymsci.2014.10.001 (2015).

of $10^{6}[138]$. These exceptional performances are due to the enhanced crystalline order obtained in these nanofibers, to their very low density of structural defects, and to the peculiar self-assembly configuration of molecules in the nanostructures. Differently to the most of other systems, cyclopentadithiophene-benzothiadiazole in nanofibers present $\pi$-stacking in a direction perpendicular to the fiber longitudinal axis as schematized in Fig. 28a, and backbones aligned along the fiber length, which can further favor charge transport. In the resulting device, an individual fiber can be contacted with Pt electrodes fabricated by a focused ion beam (Fig. 28b,c). In another, very recent work, FET devices based on individual nanowires of a diketopyrrolopyrrole-bearing copolymer have allowed researchers to measure current on/off ratios of $10^{4}$, and hole mobilities as high as $6.0-7.0 \mathrm{~cm}^{2} / \mathrm{V} \mathrm{s}[298]$.

Concerning $n$-type nanofiber FETs, devices made by individual BBL nanobelts or BBL nanobelt networks have been found to show electron mobility, $\mu_{\mathrm{E}}$, of $(3-7) \times 10^{-3} \mathrm{~cm}^{2} / \mathrm{V} \mathrm{s}$ and current on/off ratios of $10^{4}$, and these devices are stable for many months under ambient laboratory conditions [144].

Polymer nanofibers, obtained by a soft lithographically-controlled wetting method and made of an ambipolar diketopyrrolopyrrole-benzothiadiazole-based copolymer, have been also employed as building blocks of inverter circuits [283]. The copolymer molecular structure and schematics of the device fabrication method are shown in Fig. 29a. The corresponding electrical characteristics of the inverter are displayed in Fig. 29b,c. The achieved gain is of 105, which is more than three times higher than values found in devices with other ambipolar polymers, and comparable with performances reported by single crystals. Complementary inverter arrays have been also fabricated by using aligned P3HT-based nanowires, and $n$-type nanowires based on poly $\left\{\left[N, N_{0}\right.\right.$-bis $(2$ octyldodecyl)-naphthalene-1,4,5,8-bis(dicarboximide)-2, 6-diyl]-alt-5,5'-(2,2'-bithiophene)\}, which is a $n$-type semiconducting polymer [49]. These inverters have shown dynamic switching behavior as well as switching characteristics with gain of about 17. Another fundamental class of devices 
Published in Progress in Polymer Science 43, 48-95, doi: 10.1016/j.progpolymsci.2014.10.001 (2015).

which can be realized through the development of nanofiber-based FETs is given by flexible, nonvolatile flash transistor memories. These have been demonstrated on polyethylene naphthalate substrates, exploiting electrospun P3HT fibers doped by functionalized Au nanoparticles as active material, as schematized in Fig. 30a [299]. The proposed working mechanism relies on charge trapping in the nanoparticles, enabling programming and erasing functions through the resulting low-conducting or high-conducting states, respectively. These devices can be used with an operation voltage of $\pm 5 \mathrm{~V}$, threshold bias values for shifting are in the range of 3.5-10.6 V, and data retention is longer than $10^{4} \mathrm{~s}$ (Fig. 30b,c). In addition, they can be bent due to the flexibility of both the substrate and the spun fiber.

Finally, as introduced in Sec. 4.1, from nanofiber-based FETs one can derive organic light-emitting transistors. In particular, the formation of ordered polymer fibers made of poly(3,6dialkylthieno[3,2-b]thiophene-co-bithiophene) has allowed Hsu et al. to increase the charge-carrier mobility in the polymer, and consequently to enhance hole injection into another, light-emitting conjugated polymer [300]. This has led to a significant improvement of the photon emission, with brightness values approaching $9500 \mathrm{~cd} / \mathrm{m}^{2}$.

\subsection{Energy nano-generators}

Among various physical properties and functionalities enabling the generation of electrical energy, polymer nanofibers may exhibit significant piezoelectric and thermoelectric features. As well known, the electrical properties of piezoelectric polymers are not related to charge transport as in conductive macromolecules, but instead to the presence of non-centrosymmetric phases in which a voltage bias is generated as a consequence of a deformation of the involved materials. These characteristics make such compounds suitable for converting mechanical energy to electricity. Given the importance of energy harvesting devices in many modern technologies, shaping piezoelectric polymers as nanofibers, potentially embeddable in miniaturized generators and in smart textiles, has rapidly attracted the interest of many researchers. In particular, in the last decade 
Published in Progress in Polymer Science 43, 48-95, doi: 10.1016/j.progpolymsci.2014.10.001 (2015).

several reports have been published on the realization of spun piezoelectric nanofibers made of PVDF or of its derivative poly(vinylidenefluoride-co-trifluoroethylene) [P(VDF-TrFe)] [114, 215, 286, 301-319]. These polymers exhibit ferroelectric properties in their crystalline $\beta$-phase, due to electric dipoles related to hydrogen and fluorine atoms in the direction normal to the carbon backbones. In conventional materials and films, such properties have been normally obtained following electrical poling, or following mechanical stretching performed after synthesis and fabrication stages. Instead, various evidences have been reported, that techniques for producing nanofibers, and especially electrospinning methods, may induce ferroelectric properties in-situ during fiber drawing, thus making the a-posteriori poling unnecessary.

For example, randomly oriented PVDF nanofibers have been demonstrated to show an increasing content of the $\beta$-phase upon increasing the voltage bias used in electrospinning [316]. In addition, generators made by these nanofibers have been used to drive a Peltier cooler (Fig. 31a-k). Hence the combination of these devices may lead to mechanically-powered cooling textiles, possibly realized on large areas by suitable variants of the electrospinning method which show high throughput (Fig. 311). Similarly, the reduction of the nanofiber diameter has been found to be related with an increase of the $\beta$-phase content $[305,313]$. Such an increase has also been observed following the addition of $\mathrm{Ni}_{0.5} \mathrm{Zn}_{0.5} \mathrm{Fe}_{2} \mathrm{O}_{4}$ nanoparticles [306], of carbon nanotubes [307], or of the hydrated salt, nickel chloride hexahydrate, into the fibers [315]. Nano-generators based on PVDF-based piezoelectric nanofibers have been reported to be highly efficient in terms of conversion of elastic strain energy to electrical energy, with values of the energy conversion efficiency exceeding $20 \%$ [215].

As valuable alternative to PVDF polymers, the rod-like liquid crystalline poly $(\gamma$-benzyl $\alpha, \mathrm{L}-$ glutamate) (PBLG) has been electrospun and found to form piezoelectric nanofibers [320]. The authors have proposed that in this case achieving a polarity along the fiber longitudinal axis is made 
Published in Progress in Polymer Science 43, 48-95, doi: 10.1016/j.progpolymsci.2014.10.001 (2015).

easier compared to PVDF polymers, due to the particular, $\alpha$-helical conformation of poly $(\gamma$-benzyl

$\alpha$, L-glutamate).

Various device geometries have been proposed for using piezoelectric polymer nanofibers, including both vertical architectures in which nanofibers are sandwiched between electrodes (working by compression) [310], and planar configurations more suitable for stretching/release [215] and bending/release [114, 308] experiments (Fig. 32). Of course, such versatility, together with the extremely sensitive response of nanofibers to deformations, has made these devices useful not only for energy harvesting, but also for force, acceleration, strain and pressure sensors [114, 314]. In particular, large-area sheets of $\mathrm{P}(\mathrm{VDF}-\mathrm{TrFe})$ electrospun nanofibers have been employed to produce flexural sensors, and devices able to detect pressures as low as $0.1 \mathrm{~Pa}$ [114] (Fig. 33). In addition, in this work the piezoelectric behavior of polymer nanofibers has been modeled in detail. Thermoelectricity is an interesting alternative to fabricate polymer sources of renewable energy. The thermoelectric power is generated by a thermal gradient across a material exhibiting the socalled Seeback effect. Conjugated polymers, showing good transport properties and thermal conductivity much lower than metals, can be promising candidates for realizing thermoelectric generators [321]. However, the related measurements are experimentally quite complex. Recently, a study of the thermoelectric properties of various PEDOT samples has highlighted the significant dependence of the Seeback coefficient, $S$, on the molecular organization, as well as the important role played by bipolarons in this framework [322]. For this reason, conjugated polymer nanofibers, nanowires and nanotubes could be especially intriguing, due to their frequent higher molecular order and improved charge-carrier transport compared to more conventional structures such as thin films. Applications of cheap, thermoelectric systems based on organic fibers may be easily envisaged in waste heat capture in wearable electronics and smart textiles [323]. A few pioneering experiments have been reported on the thermoelectric properties of conjugated polymer nanofibers and nanotubes. 
Published in Progress in Polymer Science 43, 48-95, doi: 10.1016/j.progpolymsci.2014.10.001 (2015).

In 2010, PAN nanotubes doped by $\beta$-naphthalene sulfonic acid with a 4:1 ratio have been shown to have a Seeback coefficient of $212.4 \mu \mathrm{V} / \mathrm{K}$, namely about seven times higher than that of a reference sample without nanostructure at $300 \mathrm{~K}$ [324]. Interestingly, this promising result has been related to the concomitant increase of the electrical conductivity and decrease of the thermal conductivity $(0.21 \mathrm{~W} / \mathrm{mK})$ with respect to the reference. Both these effects can be attributed to the elongated and nanostructured shape of the conductive medium. As a consequence, the thermoelectric power factor, given by $S^{2}$ times the electrical conductivity, has been found to increase by two orders of magnitude with respect to the reference.

In 2011, Taggart et al. have compared $200 \mu \mathrm{m}$-long PEDOT nanowires, obtained by electropolymerizing the corresponding monomer, and thin films in terms of their thermoelectric performances [325]. The fabricated devices have included embedded heaters and thermocouples (Fig. 34). At $310 \mathrm{~K}$, the nanowires have been found to exhibit a Seeback coefficient of $122 \mu \mathrm{V} / \mathrm{K}$, which is significantly higher than the value found for films $(57 \mu \mathrm{V} / \mathrm{K})$. The enhanced Seeback coefficient can be mainly attributed to the relatively lower carrier concentration, in agreement with the Mott equation:

$$
S=\frac{\pi^{2} k^{2} m * T}{\left(3 \pi^{2}\right)^{2 / 3} \hbar e n_{e}^{2 / 3}},
$$

where $k$ is the Boltzmann constant, $\hbar$ is the Planck constant divided by $2 \pi, T$ is the absolute temperature, $m^{*}$ indicates the effective mass of majority carriers, $e$ is the charge and $n_{e}$ is the carrier concentration. In addition, notwithstanding such lower carrier concentration, the electrical conductivity in nanowires is still higher than that in films, due to the significantly higher mobility. Finally, in 2012 enhanced thermoelectric properties have been found in composite nanofibers made of carbon nanotubes and PAN, and fabricated by electrospinning and in-situ polymerization [326]. 
Published in Progress in Polymer Science 43, 48-95, doi: 10.1016/j.progpolymsci.2014.10.001 (2015).

\section{Actuators}

Many conjugated and shape-memory polymers can be used to realize actuators. Nanofibers realized by these compounds are especially attractive materials in this respect, due to their ultra-high surface to volume ratio (making them better controllable by chemical stimuli or through light or heat excitation) and to their elongated shape (resembling muscular tissues, tendons, and rods at the micro-scales). Other possible applications include micro-robotics, micro-tweezers, biologicallyinspired microelectro-mechanical systems, and valves for microfluidic circuits [327]. Another class of interesting materials in this respect is given by piezoelectric polymers forming nanofibers and used for actuation following the application of electric fields [309]. Various examples of piezoelectric nanofibers have been reviewed in the previous Section.

More in general, externally applied electric or magnetic fields can be used to induce mechanical actuation in a lot of suitable polymers or blends. To this aim, electrospun polycaprolactone nanofibers have been filled by multiwalled carbon nanotubes coated by $\mathrm{F}_{3} \mathrm{O}_{4}$ nanoparticles, making the nanostructures responsive to magnetic fields [328]. This has allowed remotely-controlled shape memory effects to be found in the hybrid nanofibers. Also some hydrogel fibers have been found to be electro-active, and controllable with relatively modest electric fields (500 $\left.\mathrm{V} \mathrm{m}^{-1}\right)$ [329], or by means of changes of $\mathrm{pH}$ [330-333]. In these systems as well as in conjugated polymers, the mechanisms at the base of actuation are mainly due to reversible swelling. This effect is in turn related to the embedment of dopant ions and solvent molecules. For instance, flash-welding of PAN nanofibers has allowed films to be obtained, which have shown rapid reversible actuation (bending and curling) following the interaction with aqueous acids and bases [334]. The flash-welding is a photo-thermal process, in which the high energy provided by incoming light leads the top surface of polymer nanostructures to melt, and may induce further cross-linking. The resulting asymmetric films and components have been demonstrated to be stable, and do not undergo delamination. Photographs of the films during actuation, together with sketches of their working principle, are shown in Fig. 35. An unprecedented $\left(>720^{\circ}\right)$ bending has been obtained in this way. In the last 
Published in Progress in Polymer Science 43, 48-95, doi: 10.1016/j.progpolymsci.2014.10.001 (2015).

years, flash welding has been also applied to nanocomposite films of PVA embedding PAN nanofibers to realize actuators [335].

Another approach has been based on wet spinning of chitosan, followed by in-situ polymerization of PAN [336] or of PPY [337]. Both $\mathrm{pH}$ variations and electric stimuli have been used to induce actuation in the resulting fibers. Liquid crystalline elastomers have also been processed through a wet spinning set-up, assisted by UV irradiation to promote cross-linking, and used to realize polymer filaments which exhibit reversible actuation following phase transitions in the liquid crystal (Fig. 36a-c) [338]. Other works have been focused on the electromechanical bending of electrospun PVA nanofibers followed by in-situ PAN polymerization [339], of cellulose acetate nanofibers embedding chopped PAN nanoparticles [314], of coaxially electrospun polycaprolactone, multi-walled carbon nanotubes, and poly(acrylic acid)/PVA [340], of polymer microfibers made from an ethoxysilane derivative of redox active polyferrocenylmethylvinylsilane (Fig. 36d,e) [341], and so on. In particular, strain induced by external stimuli have been found to occur rapidly, with response times of the order of $10 \mathrm{~ms}$ following electrochemical oxidation of the polymer fibers [341], which is very promising in view of the realization of real-time adaptive systems.

\section{Conclusions and perspectives}

The applications of active polymer nanofibers range from optoelectronics to energy generation, from electronic components to micromechanical systems. All of these are based on the versatility and on the tight control on molecular order, composition, and fiber geometry which have been made possible by the various technologies developed in the last years for the fabrication of these nanostructures. This review has presented the recent advancements of research on active polymer nanofibers, with a focus on important efforts performed in the last decade and on the criteria and requirements which have made this class of nanomaterials so attractive and useful for a so wide variety of applications. Many of the related fabrication techniques are quite unique in the broad 
Published in Progress in Polymer Science 43, 48-95, doi: 10.1016/j.progpolymsci.2014.10.001 (2015).

scenario of nanotechnologies, due to their significant perspectives of cost reduction and process upscaling.

Of course, polymer nanofibers can be useful in many fields other than opto- and nanoelectronics. Interested readers may look for excellent reviews specialized on filtration, biomedicine and tissue engineering [342-344], and complete details on these and other applications, as well as on the fundamentals of the different available fabrication and processing methods, may be found in dedicated books on polymer nanofibers $[1,2,77]$.

Importantly, spanning across polymer physics, chemistry, materials science and engineering, the investigation of fundamental properties of electronically-active and light-emitting polymer nanofibers and their embedment in devices can stimulate researchers with very different scientific backgrounds. The outlook for the integration of active nanofibers in materials and devices of practical use is increasingly bright, given that the related production technologies are becoming closer and closer to the industrial production scale [6]. On the other hand, a few important challenges still remain. For devices and applications based on individual nanofibers, especially in nanoelectronics, the capability of handling effectively the nanostructures developing smart positioning or manipulation methods, has to be greatly improved. In this respect, the integration of fiber spinning methods with high-precision and fast, controlled translation stages for printing can be certainly of help. Furthermore, for many techniques obtaining uniform nanofibers is still an issue. Improving our capabilities of further narrowing the fiber diameter distribution or of separating nanofibers of different size would be strongly beneficial to applications whose performance metrics critically depends on nanofiber transversal size. The development of novel hybrid materials involving inorganic nanoparticles in addition to polymers might greatly contribute in tailoring and ultimately enhancing various physical properties, such as nanofiber light emission, photon scattering, charge-carrier transport, and others. Finally, improving the capability of interfacing polymer nanofibers with metal and polymer electrodes, possibly within stretchable and bendable architectures, will certainly open new routes for wearable electronics. Overall, in the near future a 
Published in Progress in Polymer Science 43, 48-95, doi: 10.1016/j.progpolymsci.2014.10.001 (2015).

lot of opportunities still remain open, in order to develop new materials and new concepts of devices based on functional nanofibers, thanks to the endless possibilities given by polymer chemistry.

\section{Acknowledgements}

We acknowledge the European Research Council for supporting, under the European Union's Seventh Framework Programme (FP7/2007-2013), the ERC Starting Grant "NANO-JETS" (grant agreement n. 306357). We also acknowledge the support from the Apulia Regional Projects 'Networks of Public Research Laboratories', Wafitech (9) and M. I. T. T. (13), and the Italian Minister of University and Research through the Social Innovation Cluster SOCIAL-NANO (PAC02L6800087 , D.D. 436/Ric. 13/03/2013). We gratefully thank Miss Silvia Rizzo for her kind help in editing references and collecting editorial permissions. 
Published in Progress in Polymer Science 43, 48-95, doi: 10.1016/j.progpolymsci.2014.10.001 (2015).

\section{References}

[1] Andrady AL. Science and technology of polymer nanofibers. Hoboken: John Wiley \& Sons Inc; 2008.

[2] Pisignano D. Polymer nanofibers. Cambridge: Royal Society of Chemistry; 2013.

[3] Agarwal S, Greiner A, Wendorff JH. Functional materials by electrospinning of polymers. Prog Polym Sci 2013;38:963-91.

[4] Arinstein A, Burman M, Gendelman O, Zussman E. Effect of supramolecular structure on polymer nanofibre elasticity. Nat Nanotechnol 2007;2:59-62.

[5] Shen S, Henry A, Tong J, Zheng R, Chen G. Polyethylene nanofibres with very high thermal conductivities. Nat Nanotechnol 2010;5:251-5.

[6] Persano L, Camposeo A, Tekmen C, Pisignano D. Industrial upscaling of electrospinning and applications of polymer nanofibers: a review. Macromol Mater Eng 2013;298:504-20.

[7] Guo X, Baumgarten M, Müllen K. Designing $\pi$-conjugated polymers for organic electronics. Prog Polym Sci 2013;38:1832-908.

[8] Cannon JP, Bearden SD, Khatkhatay FM, Cook J, Zivanovic Selmic S, Gold SA. Confinementinduced enhancement of hole mobility in MEH-PPV. Synth Met 2009;159:1786-91.

[9] Babel A, Li D, Xia Y, Jenekhe SA. Electrospun nanofibers of blends of conjugated polymers: morphology, optical properties, and field-effect transistors. Macromolecules 2005;38:4705-11. [10] Balderas U, Falcony C, Moggio I, Arias E, Mondragón M. A photoluminescence study of electrospun fibers of conjugated poly[2-methoxy-5-(2'-ethylhexyloxy)-1,4-phenylenevinylene] blended with poly(9-vinylcarbazole). Polymer 2013;54:2062-6.

[11] Di Camillo D, Fasano V, Ruggieri F, Santucci S, Lozzi L, Camposeo A, Pisignano D. Nearfield electrospinning of light-emitting conjugated polymer nanofibers. Nanoscale 2013;5:11637-42. 
Published in Progress in Polymer Science 43, 48-95, doi: 10.1016/j.progpolymsci.2014.10.001 (2015).

[12] Li D, Babel A, Jenekhe SA, Xia Y. Nanofibers of conjugated polymers prepared by electrospinning with a two-capillary spinneret. Adv Mater 2004;16:2062-6.

[13] Pagliara S, Camposeo A, Polini A, Cingolani R, Pisignano D. Electrospun light-emitting nanofibers as excitation source in microfluidic devices. Lab Chip 2009;9:2851-6.

[14] Di Benedetto F, Camposeo A, Pagliara S, Mele E, Persano L, Stabile R, Cingolani R, Pisignano D. Patterning of light-emitting conjugated polymer nanofibres. Nat Nanotechnol 2008;3:614-9.

[15] Zhong W, Li F, Chen L, Chen Y, Wei Y. A novel approach to electrospinning of pristine and aligned MEH-PPV using binary solvents. J Mater Chem 2012;22:5523-30.

[16] Zhou R, Chen W, Jiang X, Wang S, Gong Q. Enhanced exciton migration in electrospun poly[2-methoxy-5-(2'-ethylhexyloxy)-1,4- phenylenevinylene]/poly(vinyl pyrrolidone) nanofibers. Appl Phys Lett 2010;96:133309/1-3.

[17] Ishii Y, Murata H. True photoluminescence spectra revealed in electrospun light-emitting single nanofibers. J Mater Chem 2012;22:4695-703.

[18] Zhu Z, Zhang L, Smith S, Fong H, Sun Y, Gosztola D. Fluorescence studies of electrospun MEH-PPV/PEO nanofibers. Synth Met 2009;159:1454-9.

[19] Zhao Q, Xin Y, Huang Z, Liu S, Yang C, Li Y. Using poly[2-methoxy-5-(2'-ethyl-hexyloxy)1,4-phenylene vinylene] as shell to fabricate the highly fluorescent nanofibers by coaxial electrospinning. Polymer 2007;48:4311-5.

[20] Yin K, Zhang L, Lai C, Zhong L, Smith S, Fong H, Zhu Z. Photoluminescence anisotropy of uni-axially aligned electrospun conjugated polymer nanofibers of MEH-PPV and P3HT. J Mater Chem 2011;21:444-8.

[21] De Marco C, Mele E, Camposeo A, Stabile R, Cingolani R, Pisignano D. Organic lightemitting nanofibers by solvent-resistant nanofluidics. Adv Mater 2008;20:4158-62.

[22] Liu HA, Zepeda D, Ferraris JP, Balkus KJ. Electrospinning of poly(alkoxyphenylenevinylene) and methanofullerene nanofiber blends. ACS Appl Mater Interfaces 2009;1:1958-65. 
Published in Progress in Polymer Science 43, 48-95, doi: 10.1016/j.progpolymsci.2014.10.001 (2015).

[23] Massuyeau F, Duvail JL, Athalin H, Lorcy JM, Lefrant S, Wéry J, Faulques E. Elaboration of conjugated polymer nanowires and nanotubes for tunable photoluminescence properties. Nanotechnology 2009;20:155701/1-8.

[24] Lorcy JM, Massuyeau F, Moreau P, Chauvet O, Faulques E, Wery J, Duvail JL. Coaxial nickel/poly(p-phenylene vinylene) nanowires as luminescent building blocks manipulated magnetically. Nanotechnology 2009;20:405601/1-7.

[25] Massuyeau F, Faulques E, Athalin H, Lefrant S, Duvail JL, Wéry J, Mulazzi E, Perego R. Steady state and transient photoluminescence in poly-p-phenylene vinylene films and nanofibers. $\mathbf{J}$ Chem Phys 2009;130:124706/1-6.

[26] Zhang W, Yan E, Huang Z, Wang C, Xin Y, Zhao Q, Tong Y. Preparation and study of PPV/PVA nanofibers via electrospinning PPV precursor alcohol solution. Eur Polym J 2007;43:802-7.

[27] Xin Y, Huang Z, Li W, Jiang Z, Tong Y, Wang C. Core-sheath functional polymer nanofibers prepared by co-electrospinning. Eur Polym J 2008;44:1040-5.

[28] Xin Y, Huang Z, Yang P, Jiang Z, Wang C, Shao C. Controlling the deposition of lightemitting nanofibers/microfibers by the electrospinning of a poly( $p$-phenylene vinylene) polyelectrolyte precursor. J Appl Polym Sci 2009;114:1864-9.

[29] Anderson EB, Ingildeev D, Hermanutz F, Müller A, Schweizer M, Buchmeiser MR. Synthesis and dry-spinning fibers of sulfinyl-based poly( $p$-phenylene vinylene) (ppv) for semi-conductive textile applications. J Mater Chem 2012;22:11851-60.

[30] Fenwick O, Bozec L, Credgington D, Hammiche A, Lazzerini GM, Silberberg YR, Cacialli F. Thermochemical nanopatterning of organic semiconductors. Nat Nanotechnol 2009;4:664-8.

[31] Padron S, Patlan R, Gutierrez J, Santos N, Eubanks T, Lozano K. Production and characterization of hybrid BEH-PPV/PEO conjugated polymer nanofibers by forcespinning ${ }^{\mathrm{TM}}$. $\mathrm{J}$ Appl Polym Sci 2012;125:3610-6. 
Published in Progress in Polymer Science 43, 48-95, doi: 10.1016/j.progpolymsci.2014.10.001 (2015).

[32] Mas-Torrent M, den Boer D, Durkut M, Hadley P, Schenning APHJ. Field effect transistors based on poly(3-hexylthiophene) at different length scales. Nanotechnology 2004;15:S265-9.

[33] Samitsu S, Shimomura T, Heike S, Hashizume T, Ito K. Field-effect carrier transport in poly(3alkylthiophene) nanofiber networks and isolated nanofibers. Macromolecules 2010;43:7891-4. [34] Oosterbaan WD, Vrindts V, Berson S, Guillerez S, Douhéret O, Ruttens B, D’Haen J, Adriaensens P, Manca J, Lutsen L, Vanderzande D. Efficient formation, isolation and characterization of poly(3-alkylthiophene) nanofibres: probing order as a function of side-chain length. J Mater Chem 2009;19:5424-35.

[35] Kiriy N, Jähne E, Adler H-J, Schneider M, Kiriy A, Gorodyska G, Minko S, Jehnichen D, Simon P, Fokin AA, Stamm M. One-dimensional aggregation of regioregular polyalkylthiophenes. Nano Lett 2003;3:707-12.

[36] Samitsu S, Shimomura T, Ito K. Nanofiber preparation by whisker method using solventsoluble conducting polymers. Thin Solid Films 2008;516:2478-86.

[37] Samitsu S, Takanishi Y, Yamamoto J. Self-assembly and one-dimensional alignment of a conducting polymer nanofiber in a nematic liquid crystal. Macromolecules 2009;42:4366-8. [38] Liu J, Arif M, Zou J, Khondaker SI, Zhai L. Controlling poly(3-hexylthiophene) crystal dimension: nanowhiskers and nanoribbons. Macromolecules 2009;42:9390-3.

[39] Zhang R, Li B, Iovu MC, Jeffries-El M, Sauvé G, Cooper J, Jia S, Tristram-Nagle S, Smilgies DM, Lambeth DN, McCullough RD, Kowalewski T. Nanostructure dependence of field-effect mobility in regioregular poly(3-hexylthiophene) thin film field effect transistors. J Am Chem Soc $2006 ; 128: 3480-1$.

[40] Kim DH, Han JT, Park YD, Jang Y, Cho JH, Hwang M, Cho K. Single-crystal polythiophene microwires grown by self-assembly. Adv Mater 2006;18:719-23.

[41] Sauvé G, McCullough RD. High field-effect mobilities for diblock copolymers of poly(3hexylthiophene) and poly(methyl acrylate). Adv Mater 2007;19:1822-5. 
Published in Progress in Polymer Science 43, 48-95, doi: 10.1016/j.progpolymsci.2014.10.001 (2015).

[42] DiCarmine PM, Fokina A, Seferos DS. Solvent/electrolyte control of the wall thickness of template-synthesized nanostructures. Chem Mater 2011;23:3787-94.

[43] Wang H-S, Lin L-H, Chen S-Y, Wang Y-L, Wei K-H. Ordered polythiophene/fullerene composite core-shell nanorod arrays for solar cell applications. Nanotechnology 2009;20:075201/1-5.

[44] Goto H, Akagi K. Asymmetric electrochemical polymerization: preparation of polybithiophene in a chiral nematic liquid crystal field and optically active electrochromism. Macromolecules 2005;38:1091-8.

[45] Lee S, Moon GD, Jeong U. Continuous production of uniform poly(3-hexylthiophene) (P3HT) nanofibers by electrospinning and their electrical properties. J Mater Chem 2009;19:743-8. [46] Lee SW, Lee HJ, Choi JH, Koh WG, Myoung JM, Hur JH, Park JJ, Cho JH, Jeong U. Periodic array of polyelectrolyte-gated organic transistors from electrospun poly(3-hexylthiophene) nanofibers. Nano Lett 2010;10:347-51.

[47] Bianco A, Bertarelli C, Frisk S, Rabolt JF, Gallazzi MC, Zerbi G. Electrospun polyalkylthiophene/polyethyleneoxide fibers: optical characterization. Synth Met 2007;157:276-81. [48] Kim T, Im JH, Choi HS, Yang SJ, Kim SW, Park CR. Preparation and photoluminescence (PL) performance of a nanoweb of P3HT nanofibers with diameters below $100 \mathrm{~nm}$. J Mater Chem 2011;21:14231-9.

[49] Min S-Y, Kim T-S, Kim BJ, Cho H, Noh Y-Y, Yang H, Cho JH, Lee T-W. Large-scale organic nanowire lithography and electronics. Nat Commun 2013;4:1773/1-9. [50] Mele E, Lezzi F, Polini A, Altamura D, Giannini C, Pisignano D. Enhanced charge-carrier mobility in polymer nanofibers realized by solvent-resistant soft nanolithography. J Mater Chem 2012;22:18051-6.

[51] D’Arcy JM, Tran HD, Tung VC, Tucker-Schwartz AK, Wong RP, Yang Y, Kaner RB. Versatile solution for growing thin films of conducting polymers. Proc Natl Acad Sci USA 2010;107:19673-8. 
Published in Progress in Polymer Science 43, 48-95, doi: 10.1016/j.progpolymsci.2014.10.001 (2015).

[52] O’Brien GA, Quinn AJ, Tanner DA, Redmond G. A single polymer nanowire photodetector. Adv Mater 2006;18:2379-83.

[53] O'Carroll D, Lieberwirth I, Redmond G. Melt-processed polyfluorene nanowires as active waveguides. Small 2007;3:1178-83.

[54] O'Carroll D, Lieberwirth I, Redmond G. Microcavity effects and optically pumped lasing in single conjugated polymer nanowires. Nat Nanotechnol 2007;2:180-4.

[55] Moynihan S, Iacopino D, O’Carroll D, Lovera P, Redmond G. Template synthesis of highly oriented polyfluorene nanotube arrays. Chem Mater 2008;20:996-1003.

[56] O’Carroll D, Iacopino D, O’Riordan A, Lovera P, O’Connor E, O’Brien GA, Redmond G. Poly(9,9-dioctylfluorene) nanowires with pronounced $\beta$-phase morphology: synthesis, characterization, and optical properties. Adv Mater 2008;20:42-8.

[57] Moynihan S, Lovera P, O'Carroll D, Iacopino D, Redmond G. Alignment and dynamic manipulation of conjugated polymer nanowires in nematic liquid crystal hosts. Adv Mater 2008;20:2497-502.

[58] O'Carroll D, Iacopino D, Redmond G. Luminescent conjugated polymer nanowire Y-junctions with on-branch molecular anisotropy. Adv Mater 2009;21:1160-5.

[59] O’Riordan A, Iacopino D, Lovera P, Floyd L, Reynolds K, Redmond G. Dielectrophoretic selfassembly of polarized light emitting poly(9,9-dioctylfluorene) nanofibre arrays. Nanotechnology 2011;22:105602/1-8.

[60] Lee C-C, Lai S-Y, Su W-B, Chen H-L, Chung C-L, Chen J-H. Relationship between the microstructure development and the photoluminescence efficiency of electrospun poly $(9,9-$ dioctylfluorene-2,7-diyl) fibers. J Phys Chem C 2013;117:20387-96.

[61] Vohra V, Giovanella U, Tubino R, Murata H, Botta C. Electroluminescence from conjugated polymer electrospun nanofibers in solution processable organic light-emitting diodes. ACS Nano 2011;5:5572-8. 
Published in Progress in Polymer Science 43, 48-95, doi: 10.1016/j.progpolymsci.2014.10.001 (2015).

[62] Zheng Z, Yim KH, Saifullah MSM, Welland ME, Friend RH, Kim J-S, Huck WTS. Uniaxial alignment of liquid-crystalline conjugated polymers by nanoconfinement. Nano Lett 2007;7:98792.

[63] Wang Y, Park JS, Leech JP, Miao S, Bunz UHF. Poly(aryleneethynylene)s with orange, yellow, green, and blue solid-state fluorescence. Macromolecules 2007;40:1843-50.

[64] Kyotani M, Matsushita S, Goh M, Nagai T, Matsui Y, Akagi K. Entanglement-free fibrils of aligned polyacetylene films that produce single nanofibers. Nanoscale 2010;2:509-14.

[65] Goh M, Matsushita S, Akagi K. From helical polyacetylene to helical graphite: synthesis in the chiral nematic liquid crystal field and morphology-retaining carbonization. Chem Soc Rev 2010;39:2466-76.

[66] Mori T, Kyotani M, Akagi K. Horizontally and vertically aligned helical conjugated polymers: comprehensive formation mechanisms of helical fibrillar morphologies in orientation-controlled asymmetric reaction fields consisting of chiral nematic liquid crystals. Chem Sci 2011;2:1389-5. [67] Camposeo A, Di Benedetto F, Stabile R, Cingolani R, Pisignano D. Electrospun dye-doped polymer nanofibers emitting in the near infrared. Appl Phys Lett 2007;90:143115/1-3.

[68] Camposeo A, Di Benedetto F, Stabile R, Neves AAR, Cingolani R, Pisignano D. Laser emission from electrospun polymer nanofibers. Small 2009;5:562-6.

[69] Moran-Mirabal JM, Slinker JD, DeFranco JA, Verbridge SS, Ilic R, Flores-Torres S, Abruña H, Malliaras GG, Craighead HG. Electrospun light-emitting nanofibers. Nano Lett 2007;7:458-63. [70] Dhakal KP, Lee H, Lee JW, Joo J, Guthold M, Kim J. Electrospinning and optical characterization of organic rubrene nanofibers. J Appl Phys 2012;111:123504/1-6.

[71] Campoy-Quiles M, Ishii Y, Sakai H, Murata H. Highly polarized luminescence from aligned conjugated polymer electrospun nanofibers. Appl Phys Lett 2008;92:213305/1-3.

[72] Chen M-C, Sun Y-C, Chen Y-H. Electrically conductive nanofibers with highly oriented structures and their potential application in skeletal muscle tissue engineering. Acta Biomater 2013;9:5562-72. 
Published in Progress in Polymer Science 43, 48-95, doi: 10.1016/j.progpolymsci.2014.10.001 (2015).

[73] Greiner A, Wendorff JH. Electrospinning: a fascinating method for the preparation of ultrathin fibers. Angew Chem Int Ed 2007;46:5670-3.

[74] Li D, Xia Y. Electrospinning of nanofibers: reinventing the wheel? Adv Mater 2004;16:115170.

[75] Kim FS, Ren G, Jenekhe SA. One-dimensional nanostructures of $\pi$-conjugated molecular systems: assembly, properties, and applications from photovoltaics, sensors, and nanophotonics to nanoelectronics. Chem Mater 2011;23:682-732.

[76] Long Y-Z, Li M-M, Gu C, Wan M, Duvail J-L, Liu Z, Fan Z. Recent advances in synthesis, physical properties and applications of conducting polymer nanotubes and nanofibers. Prog Polym Sci 2011;36:1415-42.

[77] Wendorff JH, Agarwal S, Greiner A. Electrospinning. Materials, Processing and Applications. Weihneim: Wiley-VCH; 2012.

[78] Huang C, Chen S, Lai C, Reneker DH, Qiu H, Ye Y, Hou H. Electrospun polymer nanofibres with small diameters. Nanotechnology 2006;17:1558-63.

[79] Elhadj S, Rioux RM, Dickey MD, DeYoreo JJ, Whitesides GM. Subnanometer replica molding of molecular steps on ionic crystals. Nano Lett 2010;10:4140-5.

[80] Gates BD, Xu Q, Stewart M, Ryan D, Willson CG, Whitesides GM. New approaches to nanofabrication: molding, printing, and other techniques. Chem Rev 2005;105:1171-96.

[81] Bao Q, Zhang H, Yang J-X, Wang S, Tang DY, Jose R, Ramakrishna S, Lim CT, Loh KP. Graphene-polymer nanofiber membrane for ultrafast photonics. Adv Funct Mater 2010; 20:782-91. [82] Liu H, Edel JB, Bellan LM, Craighead HG. Electrospun Polymer Nanofibers as Subwavelength Optical Waveguides Incorporating Quantum Dots. Small 2006;2:495-9.

[83] Meng C, Xiao Y, Wang P, Zhang L, Liu Y, Tong L. Quantum-dot-doped polymer nanofibers for optical sensing. Adv Mater 2011;23:3770-4.

[84] Gu F, Yu H, Wang P, Yang Z, Tong L. Light-emitting polymer single nanofibers via waveguiding excitation. ACS Nano 2010;4:5332-8. 
Published in Progress in Polymer Science 43, 48-95, doi: 10.1016/j.progpolymsci.2014.10.001 (2015).

[85] Wang P, Li Z, Zhang L, Tong L. Electron-beam-activated light-emitting polymer nanofibers. Opt Lett 2013;38:1040-2.

[86] Fasano V, Polini A, Morello G, Moffa M, Camposeo A, Pisignano D. Bright light emission and waveguiding in conjugated polymer nanofibers electrospun from organic salt added solutions. Macromolecules 2013;46:5935-42.

[87] Di Benedetto F, Mele E, Camposeo A, Athanassiou A, Cingolani R, Pisignano D. Photoswitchable organic nanofibers. Adv Mater 2008;20:314-8.

[88] Iacopino D, Redmond G. Reversible modulation of photoluminescence from conjugated polymer nanotubes by incorporation of photochromic spirooxazine molecules. Chem Commun $2011 ; 47: 9170-2$.

[89] Molenkamp WC, Watanabe M, Miyata H, Tolbert SH. Highly polarized luminescence from optical quality films of a semiconducting polymer aligned within oriented mesoporous silica. J Am Chem Soc 2004;126:4476-7.

[90] Kuo C-C, Wang C-T, Chen W-C. Highly-aligned electrospun luminescent nanofibers prepared from polyfluorene/PMMA blends: fabrication, morphology, photophysical properties and sensory applications. Macromol Mater Eng 2008;293:999-1008.

[91] Pagliara S, Vitiello M, Camposeo A, Polini A, Cingolani R, Scamarcio G, Pisignano D. Optical anisotropy in single light-emitting polymer nanofibers. J Phys Chem C 2011;115:20399_ 405.

[92] Kwak G, Fukao S, Fujiki M, Sakaguchi T, Masuda T. Nanoporous, honeycomb-structured network fibers spun from semiflexible, ultrahigh molecular weight, disubstituted aromatic polyacetylenes: superhierarchical structure and unique optical anisotropy. Chem Mater 2006;18:5537-42.

[93] Kawabata K, Goto H. Dynamically controllable emission of polymer nanofibers: electrofluorescence chromism and polarized emission of polycarbazole derivatives. Chem Eur J 2012;18:15065-72. 
Published in Progress in Polymer Science 43, 48-95, doi: 10.1016/j.progpolymsci.2014.10.001 (2015).

[94] Labastide JA, Baghgar M, McKenna A, Barnes MD. Time- and polarization-resolved photoluminescence decay from isolated polythiophene (P3HT) nanofibers. J Phys Chem C 2012;116:23803-11.

[95] Dawson K, Lovera P, Iacopino D, O’Riordan A, Redmond G. Multi-colour emission from dye doped polymeric nanotubes by host-guest energy transfer. J Mater Chem 2011;21:15995-6000.

[96] Camposeo A, Di Benedetto F, Cingolani R, Pisignano D. Full color control and white emission from conjugated polymer nanofibers. Appl Phys Lett 2009;94:043109/1-3.

[97] Ner Y, Grote JG, Stuart JA, Sotzing GA. White luminescence from multiple-dye-doped electrospun DNA nanofibers by fluorescence resonance energy transfer. Angew Chem Int Ed 2009;48: 5134-38.

[98] Kim J, Noh J, Jo S, Park KE, Park WH, Lee TS. Simple technique for spatially separated nanofibers/nanobeads by multinozzle electrospinning toward white-light emission. ACS Appl Mater Interfaces 2013;5:6038-44.

[99] Wang C-T, Kuo C-C, Chen H-C, Chen W-C. Non-woven and aligned electrospun multicomponent luminescent polymer nanofibers: effects of aggregated morphology on the photophysical properties. Nanotechnology 2009;20:375604/1-10.

[100] de Melo EF, Santana NdC, Bezerra Alves KG, de Sá GF, de Melo CP, Rodrigues MO, Júnior SA. LnMOF@PVA nanofiber: energy transfer and multicolor light-emitting devices. J Mater Chem C 2013;1:7574-81.

[101] Li S, Zhang K. Electrospinning composite fibers doped with rhenium complex: preparation, characterization and photophysical feature comparison. J Lumin 2014;147:134-40.

[102] Sui XM, Shao CL, Liu YC. White-light emission of polyvinyl alcohol/ZnO hybrid nanofibers prepared by electrospinning. Appl Phys Lett 2005;87:113115/1-3.

[103] Sun H, Zhang H, Zhang J, Wei H, Ju J, Li M, Yang B. White-light emission nanofibers obtained from assembling aqueous single-colored CdTe NCs into a PPV precursor and PVA matrix. J Mater Chem 2009;19:6740-4. 
Published in Progress in Polymer Science 43, 48-95, doi: 10.1016/j.progpolymsci.2014.10.001 (2015).

[104] Wang C, Yan E, Li G, Sun Z, Wang S, Tong Y, Li W, Xin Y, Huang Z, Yan P. Tunable photoluminescence of poly(phenylene vinylene) nanofibers by doping of semiconductor quantum dots and polymer. Synth Met 2010;160:1382-6.

[105] Vohra V, Devaux A, Dieu L-Q, Scavia G, Catellani M, Calzaferri G, Botta C. Energy transfer in fluorescent nanofibers embedding dye-loaded zeolite L crystals. Adv Mater 2009;21:1146-50. [106] Vohra V, Calzaferri G, Destri S, Pasini M, Porzio W, Botta C. Toward white light emission through efficient two-step energy transfer in hybrid nanofibers. ACS Nano 2010;4:1409-16.

[107] Giansante C, Schäfer C, Raffy G, Del Guerzo A. Exploiting direct and cascade energy transfer for color-tunable and white-light emission in three-component self-assembled nanofibers. J Phys Chem C 2012;116:21706-16.

[108] Garreau A, Massuyeau F, Cordier S, Molard Y, Gautron E, Bertoncini P, Faulques E, Wery J, Humbert B, Bulou A, Duvail J-L. Color control in coaxial two-luminophore nanowires. ACS Nano $2013 ; 7: 2977-87$.

[109] Lu G, Tang H, Qu Y, Li L, Yang X. Enhanced electrical conductivity of highly crystalline polythiophene/insulating-polymer composite. Macromolecules 2007;40:6579-84.

[110] Qiu L, Lee WH, Wang X, Kim JS, Lim JA, Kwak D, LeeS, Cho K. Organic thin-film transistors based on polythiophene nanowires embedded in insulating polymer. Adv Mater 2009;21:1349-53.

[111] Sun B, Long Y-Z, Chen Z-J, Liu S-L, Zhang H-D, Zhang J-C, Han W-P. Recent advances in flexible and stretchable electronic devices via electrospinning. J Mater Chem C 2014;2:1209-19. [112] Laforgue A. Electrically controlled colour-changing textiles using the resistive heating properties of PEDOT nanofibers. J Mater Chem 2010;20:8233-5.

[113] Zhan Y, Mei Y, Zheng L. Materials capability and device performance in flexible electronics for the internet of things. J Mater Chem C 2014;2:1220-32. 
Published in Progress in Polymer Science 43, 48-95, doi: 10.1016/j.progpolymsci.2014.10.001 (2015).

[114] Persano L, Dagdeviren C, Su Y, Zhang Y, Girardo S, Pisignano D, Huang Y, Rogers JA. High performance piezoelectric devices based on aligned arrays of nanofibers of poly(vinylidenefluoride-co-trifluoroethylene). Nat Commun 2013;4:1633/1-10.

[115] Mannarino MM, Rutledge GC. Mechanical and tribological properties of electrospun PA 6(3)T fiber mats. Polymer 2012;53:3017-25.

[116] Park S-I, Xiong Y, Kim R-H, Elvikis P, Meitl M, Kim D-H, Wu J, Yoon J, Yu C-J, Liu Z, Huang Y, Hwang K-C, Ferreira P, Li X, Choquette K, Rogers JA. Printed assemblies of inorganic light-emitting diodes for deformable and semitransparent displays. Science 2009;325:977-81.

[117] Sekitani T, Noguchi Y, Hata K, Fukushima T, Aida T, Someya T. A rubberlike stretchable active matrix using elastic conductors. Science 2008;321:1468-72.

[118] Benight SJ, Wang C, Tok JBH, Bao Z. Stretchable and self-healing polymers and devicesfor electronic skin. Prog Polym Sci 2013;38:1961-77.

[119] Park M, Park J, Jeong U. Design of conductive composite elastomers for stretchable electronics. Nano Today 2014;9:244-60.

[120] Zhao X, Wang Q. Harnessing large deformation and instabilities of soft dielectrics: theory, experiment, and application. Appl Phys Rev 2014;1: 021304/1-24.

[121] Shin M, Song JH, Lim G-H, Lim B, Park J-J, Jeong U. Highly stretchable polymer transistors consisting entirely of stretchable device components. Adv Mater 2014;26:3706-11.

[122] Oh JY, Shin M, Lee JB, Ahn J-H, Baik HK, Jeong U. Effect of PEDOT nanofibril networks on the conductivity, flexibility, and coatability of PEDOT:PSS films. ACS Appl Mater Interfaces 2014;6:6954-61.

[123] Oh JY, Shin M, Lee TI, Jang WS, Lee Y-J, Kim CS, Kang J-W, Myoung J-M, Baik HK, Jeong U. Highly bendable large-area printed bulk heterojunction film prepared by the self-seeded growth of poly(3-hexylthiophene) nanofibrils. Macromolecules 2013;46:3534-43.

[124] Rahy A, Yang DJ. Synthesis of highly conductive polyaniline nanofibers. Mater Lett $2008 ; 62: 4311-4$. 
Published in Progress in Polymer Science 43, 48-95, doi: 10.1016/j.progpolymsci.2014.10.001 (2015).

[125] Li D, Huang J, Kaner RB. Polyaniline nanofibers: a unique polymer nanostructure for versatile applications. Acc Chem Res 2009;42:135-45.

[126] Bhadra S, Khastgir D, Singha NK, Lee JH. Progress in preparation, processing and applications of polyaniline. Prog Polym Sci 2009;34:783-810.

[127] Huang J, Kaner RB. The intrinsic nanofibrillar morphology of polyaniline. Chem Commun 2006;4:367-76.

[128] Pramanik S, Karak N, Banerjee S, Kumar A. Effects of solvent interactions on the structure and properties of prepared PAni nanofibers. J Appl Polym Sci 2012;126:830-6.

[129] Bozdag KD, Chiou N-R, Prigodin VN, Epstein AJ. Anomalous charge transport in multiple interconnected polyaniline nanofibers. Org Electron 2013;14:1419-23.

[130] Yan Y, Fang J, Liang J, Zhang Y, Wei Z. Helical heterojunctions originating from helical inversion of conducting polymer nanofibers. Chem Commun 2012;48:2843-5.

[131] Ornatska M, Peleshanko S, Genson KL, Rybak B, Bergman KN, Tsukruk VV. Assembling of amphiphilic highly branched molecules in supramolecular nanofibers. J Am Chem Soc 2004;126:9675-84.

[132] Merlo JA, Frisbie CD. Field effect conductance of conducting polymer nanofibers. J Polym Sci Part B Polym Phys 2003;41:2674-80.

[133] Merlo JA, Frisbie CD. Field effect transport and trapping in regioregular polythiophene nanofibers. J Phys Chem B 2004;108:19169-79.

[134] Kim DH, Park DY, Jang Y, Kim S, Cho K. Solvent vapor-induced nanowire formation in poly(3-hexylthiophene) thin films. Macromol Rapid Commun 2005;26:834-9.

[135] Kim DH, Jang Y, Park YD, Cho K. Controlled one-dimensional nanostructures in poly(3hexylthiophene) thin film for high-performance organic field-effect transistors. J Phys Chem B 2006;110:15763-8. 
Published in Progress in Polymer Science 43, 48-95, doi: 10.1016/j.progpolymsci.2014.10.001 (2015).

[136] Grenier CRG, Pisula W, Joncheray TJ, Müllen K, Reynolds JR. Regiosymmetric poly(dialkylphenylenedioxythiophene)s: electron-rich, stackable p-conjugated nanoribbons. Angew Chem Int Ed 2007;46:714-7.

[137] Simmonds H, Bennett N, Elliott M, Macdonald E. Self-assembled organic nanowires: a structural and electronic study. J Vac Sci Technol B 2009;27:831-5.

[138] Wang S, Kappl M, Liebewirth I, Müller M, Kirchhoff K, Pisula W, Müllen K. Organic fieldeffect transistors based on highly ordered single polymer fibers. Adv Mater 2012;24:417-20.

[139] Niles ET, Roehling JD, Yamagata H, Wise AJ, Spano FK, Moulé AJ, Grey JK. J-aggregate behavior in poly-3-hexylthiophene nanofibers. J Phys Chem Lett 2012;3:259-63.

[140] Martin TP, Wise AJ, Busby E, Gao J, Roehling JD, Ford MJ, Larsen DS, Moulé AJ, Grey JK. Packing dependent electronic coupling in single poly(3-hexylthiophene) $\mathrm{H}$ - and J-aggregate nanofibers. J Phys Chem B 2013;117:4478-87.

[141] Baghgar M, Labastide JA, Bokel F, Hayward RC, Barnes MD. Effect of polymer chain folding on the transition from $\mathrm{H}$ - to J-aggregate behavior in P3HT nanofibers. J Phys Chem C 2014;118:2229-35.

[142] Roehling JD, Arslan I, Moulé AJ. Controlling microstructure in poly(3-hexylthiophene) nanofibers. J Mater Chem 2012;22:2498-506.

[143] Gao J, Kamps A, Park S-J, Grey JK. Encapsulation of poly(3-hexylthiophene) J-aggregate nanofibers with an amphiphilic block copolymer. Langmuir 2012;28:16401-7.

[144] Briseno AL, Mannsfeld SCB, Shamberger PJ, Ohuchi FS, Bao Z, Jenekhe SA, Xia Y. Selfassembly, molecular packing, and electron transport in n-type polymer semiconductor nanobelts. Chem Mater 2008;20:4712-9.

[145] Wu P-T, Ren G, Li C, Mezzenga R, Jenekhe SA. Crystalline diblock conjugated copolymers: synthesis, self-assembly, and microphase separation of poly(3-butylthiophene)-b-poly(3octylthiophene). Macromolecules 2009;42:2317-20. 
Published in Progress in Polymer Science 43, 48-95, doi: 10.1016/j.progpolymsci.2014.10.001 (2015).

[146] Lee E, Hammer B, Kim J-K, Page Z, Emrick T, Hayward RC. Hierarchical helical assembly of conjugated poly(3-hexylthiophene)-block-poly(3-triethylene glycol thiophene) diblock copolymers. J Am Chem Soc 2011;133:10390-3.

[147] Kamps AC, Fryd M, Park S-J. Hierarchical self-assembly of amphiphilic semiconducting polymers into isolated, bundled, and branched nanofibers. ACS Nano 2012;6:2844-52.

[148] He F, Gädt T, Manners I, Winnik MA. Fluorescent "barcode” multiblock co-micelles via the living self-assembly of di- and triblock copolymers with a crystalline core-forming metalloblock. J Am Chem Soc 2011;133:9095-103.

[149] Patra SK, Ahmed R, Whittell GR, Lunn DJ, Dunphy EL, Winnik MA, Manners I. Cylindrical micelles of controlled length with a $\pi$-conjugated polythiophene core via crystallization-driven selfassembly. J Am Chem Soc 2011;133:8842-5.

[150] Goto H. Electrochemical polymerization of pyrrole in cholesteric liquid crystals: morphology and optical properties. J Polym Sci Part A Polym Chem 2007;45:1377-87.

[151] Goto H, Kawabata K. Visualization of nematic liquid crystal director by alignment of $\pi$ conjugated polymer nanotubes. Macromolecules 2008;41:4551-4.

[152] Goto H, Nimori S. Liquid crystal electropolymerisation under magnetic field and resultant linear polarised electrochromism. J Mater Chem 2010;20:1891-8.

[153] Lee JI, Cho SH, Park S-M, Kim JK, Kim JK, Yu J-W, Kim YC, Russell TP. Highly aligned ultrahigh density arrays of conducting polymer nanorods using block copolymer templates. Nano Lett 2008;8:2315-20.

[154] Li F, Martens AA, Aslund A, Konradsson P, de Wolf FA, Cohen Stuart MA, Sudhölter EJR, Marcelis ATM, Leermakers FAM. Formation of nanotapes by co-assembly of triblock peptide copolymers and polythiophenes in aqueous solution. Soft Matter 2009;5:1668-73.

[155] Yoon H, Chang M, Jang J. Formation of 1D poly(3,4-ethylenedioxythiophene) nanomaterials in reverse microemulsions and their application to chemical sensors. Adv Funct Mater $2007 ; 17: 431-6$. 
Published in Progress in Polymer Science 43, 48-95, doi: 10.1016/j.progpolymsci.2014.10.001 (2015).

[156] Anilkumar P, Jayakannan M. Self-assembled cylindrical and vesicular molecular templates for polyaniline nanofibers and nanotapes. J Phys Chem B 2009;113:11614-24.

[157] Zhang X, Goux WJ, Manohar SK. Synthesis of polyaniline nanofibers by “nanofiber seeding”. J Am Chem Soc 2004;126:4502-3.

[158] Nair S, Hsiao E, Kim SH. Melt-welding and improved electrical conductivity of nonwoven porous nanofiber mats of poly(3,4-ethylenedioxythiophene) grown on electrospun polystyrene fiber template. Chem Mater 2009;21:115-21.

[159] Laforgue A, Robitaille L. Deposition of ultrathin coatings of polypyrrole and poly(3,4ethylenedioxythiophene) onto electrospun nanofibers using a vapor-phase polymerization method. Chem Mater 2010;22:2474-80.

[160] Laforgue A, Robitaille L. Production of conductive PEDOT nanofibers by the combination of electrospinning and vapor-phase polymerization. Macromolecules 2010;43:4194-200.

[161] Wang W, Li Z, Jiang T, Zhao Z, Li Y, Wang Z, Wang C. Sulfonated poly(ether ether ketone)/polypyrrole core-shell nanofibers: a novel polymeric adsorbent/conducting polymer nanostructures for ultrasensitive gas sensors. ACS Appl Mater Interfaces 2012;4:6080-4. [162] Wang W, Lu X, Li Z, Li X, Xu X, Lei J, Wang C, Baughman RH, Fang S. Weak-acceptorpolyacrylonitrile/donor-polyaniline core-shell nanofibers: a novel 1D polymeric heterojunction with high photoconductive properties. Org Electron 2012;13:2319-25.

[163] Rana S, Kim SD, Cho JW. Conducting core-sheath nanofibers for electroactive shapememory applications. Polym Adv Technol 2013;24:609-14.

[164] Miao Y-E, Fan W, Chen D, Liu T. High-performance supercapacitors based on hollow polyaniline nanofibers by electrospinning. ACS Appl Mater Interfaces 2013;5:4423-8.

[165] Liang B, Qin Z, Zhao J, Zhang Y, Zhou Z, Lu Y. Controlled synthesis, core-shell structures and electrochemical properties of polyaniline/polypyrrole composite nanofibers. J Mater Chem A 2014;2:2129-35. 
Published in Progress in Polymer Science 43, 48-95, doi: 10.1016/j.progpolymsci.2014.10.001 (2015).

[166] Wang L, Hu J, Zhang H, ZhangT. Au-impregnated polyacrylonitrile (PAN)/polythiophene (PTH) core-shell nanofibers with high-performance semiconducting properties. Chem Commun 2011;47:6837-9.

[167] Kwon OS, Park SJ, Lee JS, Park E, Kim T, Park HW, You SA, Yoon H, Jang J.

Multidimensional conducting polymer nanotubes for ultrasensitive chemical nerve agent sensing. Nano Lett 2012;12:2797-802.

[168] Liao Y, Yu D-G, Wang X, Chain W, Li X-G, Hoek EMV, Kaner RB. Carbon nanotubetemplated polyaniline nanofibers: synthesis, flash welding and ultrafiltration membranes. Nanoscale $2013 ; 5: 3856-62$.

[169] Bardavid Y, Ghabboun J, Porath D, Kotylar AB, Yitzchaik S. Formation of polyaniline layer on DNA by electrochemical polymerization. Polymer 2008;49:2217-22.

[170] Pruneanu S, Al-Said SAF, Dong L, Hollis TA, Galindo MA, Wright NG, Houlton A, Horrocks BR. Self-assembly of DNA-templated polypyrrole nanowires: spontaneous formation of conductive nanoropes. Adv Funct Mater 2008;18:2444-54.

[171] Houlton A, Pike AR, Galindo MA, Horrocks BR. DNA-based routes to semiconducting nanomaterials. Chem Commun 2009;14:1797-806.

[172] Wigenius J, Björk P, Hamedi M, Aili D. Supramolecular assembly of designed $\alpha$-helical polypeptide-based nanostructures and luminescent conjugated polyelectrolytes. Macromol Biosci 2010;10:836-41.

[173] Herland A, Thomsson D, Mirzov O, Scheblykin IG, Inganäs O. Decoration of amyloid fibrils with luminescent conjugated polymers. J Mater Chem 2008;18:126-32.

[174] Björk P, Thomsson D, Mirzov O, Wigenius J, Inganäs O, Scheblykin IG. Oligothiophene assemblies defined by DNA interaction: from single chains to disordered clusters. Small 2009;5:96103. 
Published in Progress in Polymer Science 43, 48-95, doi: 10.1016/j.progpolymsci.2014.10.001 (2015).

[175] Strong V, Uribe-Romo FJ, Battson M, Kaner R. Oriented polythiophene nanofibers grown from CdTe quantum dot surfaces. Small 2012;8:1191-6.

[176] Wu C-G, Bein T. Conducting polyaniline filaments in a mesoporous channel host. Science $1994 ; 264: 1757-9$.

[177] Martin CR. Nanomaterials: a membrane-based synthetic approach. Science 1994;266:1961-6.

[178] Il Cho S, Bok Lee S. Fast electrochemistry of conductive polymer nanotubes: synthesis, mechanism, and application. Acc Chem Res 2008;41:699-707.

[179] Moynihan S, Iacopino D, O’Carroll D, Doyle H, Tanner DA, Redmond G. Emission color tuning in semiconducting polymer nanotubes by energy transfer to organo-lanthanide dopants. Adv Mater 2007;19:2474-9.

[180] Rahman A, Sanyal MK, Gangopadhayy R, De A, Das I. Evidence of a ratchet effect in nanowires of a conducting polymer. Phys Rev B 2006;73:125313/1-5.

[181] Rahman A, Sanyal MK. Observation of charge density wave characteristics in conducting polymer nanowires: possibility of Wigner crystallization. Phys Rev B 2007;76:045110/1-6. [182] Duvail JL, Rétho P, Fernandez V, Louarn G, Molinié P, Chauvet O. Effects of the confined synthesis on conjugated polymer transport properties. J Phys Chem B 2004;108:18552-6. [183] Long YZ, Duvail JL, Chen ZJ, Jin AZ, Gu CZ. Electrical properties of isolated poly(3,4ethylenedioxythiophene) nanowires prepared by template synthesis. Polym Adv Technol $2009 ; 20: 541-4$.

[184] Döbbelin M, Tena-Zaera R, Carrasco PM, Sarasua J-R, Cabañero G, Mecerreyes D. Electrochemical synthesis of poly(3,4-ethylenedioxythiophene) nanotube arrays using $\mathrm{ZnO}$ templates. J Polym Sci Part A Polym Chem 2010;48:4648-53.

[185] Mei S, Feng X, Jin Z. Polymer nanofibers by controllable infiltration of vapour swollen polymers into cylindrical nanopores. Soft Matter 2013;9:945-51.

[186] Bhattacharyya D, Yang R, Gleason KK. High aspect ratio, functionalizable conducting copolymer nanobundles. J Mater Chem 2012;22:17147-52. 
Published in Progress in Polymer Science 43, 48-95, doi: 10.1016/j.progpolymsci.2014.10.001 (2015).

[187] Park DH, Hong YK, Cho EH, Kim MS, Kim D-C, Bang J, Kim J, Joo J. Light-emitting color barcode nanowires using polymers: nanoscale optical characteristics. ACS Nano 2010;4:5155-62. [188] Massuyeau F, Zhao Y, El Mel AA, Yaya A, Geschier F, Gautron E, Lefrant S, Mevellec JY, Ewels C, Hsu C-S, Faulques E, Wéry J, Duvail JL. Improved photoconductive properties of composite nanofibers based on aligned conjugated polymer and single-walled carbon nanotubes. Nano Res 2013;6:149-58.

[189] Park S, Chung S-W, Mirkin CA. Hybrid organic-inorganic, rod-shaped nanoresistors and diodes. J Am Chem Soc 2004;126:11772-3.

[190] Guo Y, Tang Q, Liu H, Zhang Y, Li Y, Hu W, Wang S, Zhu D. Light-controlled organic/inorganic p-n junction nanowires. J Am Chem Soc 2008;130:9198-9.

[191] Reneker DH, Fong H. Polymeric nanofibers. Washington DC: American Chemical Society; 2006.

[192] Ramakrishna S, Fujihara K, Teo W-E, Lim T-C, Ma Z. An Introduction to Electrospinning and Nanofibers. Singapore: World Scientific Publishing Co; 2005.

[193] Chronakis I. Novel nanocomposites and nanoceramics based on polymer nanofibers using electrospinning process-A review. J Mater Process Technol 2005;167:283-93.

[194] He J-H, Liu Y, Mo L-F, Wan Y-Q, Xu L. Electrospun Nanofibers and Their Applications. Shawbury: iSmithers; 2008.

[195] Reneker DH, Yarin AL. Electrospinning jets and polymer nanofibers. Polymer 2008;49:2387-425.

[196] Feng C, Khulbe KC, Matsuura T. Recent progress in the preparation, characterization, and applications of nanofibers and nanofiber membranes via electrospinning/interfacial polymerization. J Appl Polym Sci 2010;115:756-76.

[197] Persano L, Camposeo A, Pisignano D. Integrated bottom-up and top-down soft lithographies and microfabrication approaches to multifunctional polymers. J Mater Chem C 2013;1:7663-80. 
Published in Progress in Polymer Science 43, 48-95, doi: 10.1016/j.progpolymsci.2014.10.001 (2015).

[198] Hou Z, Li G, Lian H, Lin J. One-dimensional luminescent materials derived from the electrospinning process: preparation, characteristics and application. J Mater Chem 2012;22:525476.

[199] Wu X-F, Salkovskiy Y, Dzenis YA. Modeling of solvent evaporation from polymer jets in electrospinning. Appl Phys Lett 2011;98:223108/1-3.

[200] Reneker DH, Yarin AL, Zussman E, Xu H. Electrospinning of nanofibers from polymer solutions and melts. Adv Appl Mech 2007;41:43-195.

[201] Greenfeld I, Arinstein A, Fezzaa K, Rafailovich MH, Zussman E. Polymer dynamics in semidilute solution during electrospinning: a simple model and experimental observations. Phys Rev E 2011;84:041806/1-9.

[202] Greenfeld I, Fezzaa K, Rafailovich MH, Zussman E. Fast X-ray phase-contrast imaging of electrospinning polymer jets: measurements of radius, velocity, and concentration. Macromolecules 2012;45:3616-26.

[203] Camposeo A, Greenfeld I, Tantussi F, Pagliara S, Moffa M, Fuso F, Allegrini M, Zussman E, Pisignano D. Local mechanical properties of electrospun fibers correlate to their internal nanostructure. Nano Lett 2013;13:5056-62.

[204] Kakade MV, Givens S, Gardner K, Lee KH, Chase DB, Rabolt JF. Electric field induced orientation of polymer chains in macroscopically aligned electrospun polymer nanofibers. J Am Chem Soc 2007;129:2777-82.

[205] Ma M, Krikorian V, Yu JH, Thomas EL, Rutledge GC. Electrospun polymer nanofibers with internal periodic structure obtained by microphase separation of cylindrically confined block copolymers. Nano Lett 2006;6:2969-72.

[206] Pai CL, Boyce MC, Rutledge GC. Morphology of porous and wrinkled fibers of polystyrene electrospun from dimethylformamide. Macromolecules 2009;42:2102-14. 
Published in Progress in Polymer Science 43, 48-95, doi: 10.1016/j.progpolymsci.2014.10.001 (2015).

[207] García LA, Arias E, Moggio I, Romero J, Ledezma A, Ponce A, Perez O. Fluorescent coresheath fibers by electrospinning of a phenyleneethynylene/poly(styrene-co-maleimide) blend. Polymer 2011;52:5326-34.

[208] Chen D, Lei S, Chen Y. A single polyaniline nanofiber field effect transistor and its gas sensing mechanisms. Sensors 2011;11:6509-16.

[209] Manuelli A, Persano L, Pisignano D. Flexible organic field-effect transistors based on electrospun conjugated polymer nanofibers with high bending stability. Org Electron 2014;15:105661.

[210] Sun D, Chang C, Li S, Lin L. Near-field electrospinning. Nano Lett 2006;6:839-42.

[211] Chang C, Limkrailassiri K, Lin L. Continuous near-field electrospinning for large area deposition of orderly nanofiber patterns. Appl Phys Lett 2008;93:123111/1-3

[212] Rinaldi M, Ruggieri F, Lozzi L, Santucci S. Well-aligned $\mathrm{TiO}_{2}$ nanofibers grown by nearfield-electrospinning. J Vac Sci Technol B 2009;27:1829-33.

[213] Hellmann C, Belardi J, Dersch R, Greiner A, Wendorff JH, Bahnmueller S. High precision deposition electrospinning of nanofibers and nanofiber nonwovens. Polymer 2009;50:1197-205. [214] Kim H-Y, Lee M, Park KJ, Kim S, Mahadevan L. Nanopottery: coiling of electrospun polymer nanofibers. Nano Lett 2010;10:2138-40.

[215] Chang C, Tran VH, Wang J, Fuh Y-K, Lin L. Direct-write piezoelectric polymeric nanogenerator with high energy conversion efficiency. Nano Lett 2010;10:726-31.

[216] Bisht GS, Canton G, Mirsepassi A, Kulinsky L, Oh S, Dunn-Rankin D, Madou MJ. Controlled continuous patterning of polymeric nanofibers on three-dimensional substrates using low-voltage near-field electrospinning. Nano Lett 2011;11:1831-7.

[217] Zhou F-L, Hubbard PL, Eichhorn SJ, Parker GJM. Jet deposition in near-field electrospinning of patterned polycaprolactone and sugar-polycaprolactone coreeshell fibres. Polymer 2011;52:3603-10. 
Published in Progress in Polymer Science 43, 48-95, doi: 10.1016/j.progpolymsci.2014.10.001 (2015).

[218] Zheng G, Li W, Wang X, Wu D, Sun D, Lin L. Precision deposition of a nanofibre by nearfield electrospinning. J Phys D Appl Phys 2010;43:415501/1-6.

[219] Wang H, Zheng G, Li W, Wang X, Sun D. Direct-writing organic three-dimensional nanofibrous structure. Appl Phys A 2011;102:457-461.

[220] Sun Z, Zussman E, Yarin AL, Wendorff JH, Greiner A. Compound core-shell polymer nanofibers by co-electrospinning. Adv Mater 2003;15:1929-32.

[221] Li D, Xia Y. Direct fabrication of composite and ceramic hollow nanofibers by electrospinning. Nano Lett 2004;4:933-8.

[222] Ma Q, Wang J, Dong X, Yu W, Liu G. Electrospinning fabrication and characterization of magnetic-upconversion fluorescent bifunctional core-shell nanofibers. J Nanopart Res $2014 ; 16: 2239 / 1-10$.

[223] Crespy D, Friedemann K, Popa A-M. Colloid-electrospinning: fabrication of multicompartment nanofibers by the electrospinning of organic or/and inorganic dispersions and emulsions. Macromol Rapid Commun 2012;33:1978-95.

[224] Li D, McCann JT, Xia Y. Use of electrospinning to directly fabricate hollow nanofibers with functionalized inner and outer surfaces. Small 2005;1:83-6.

[225] Ferraro P, Coppola S, Grilli S, Paturzo M, Vespini V. Dispensing nano-pico droplets and liquid patterning by pyroelectrodynamic shooting. Nat Nanotechnol 2010;5:429-35.

[226] Grilli S, Coppola S, Vespini V, Merola F, Finizio A, Ferraro P. 3D lithography by rapid curing of the liquid instabilities at nanoscale. Proc Natl Acad Sci USA 2011;108:15106-11. [227] Benavides RE, Jana SC, Reneker DH. Nanofibers from scalable gas jet process. ACS Macro Lett 2012;1:1032-6.

[228] Lyons J, Li C, Ko F. Melt-electrospinning part I: processing parameters and geometric properties. Polymer 2004;45:7597-603.

[229] Dalton PD, Klinkhammer K, Salber J, Klee D, Möller M. Direct in vitro electrospinning with polymer melts. Biomacromolecules 2006;7:686-90. 
Published in Progress in Polymer Science 43, 48-95, doi: 10.1016/j.progpolymsci.2014.10.001 (2015).

[230] Zhmayev E, Cho D, Joo YL. Nanofibers from gas-assisted polymer melt electrospinning. Polymer 2010;51:4140-4.

[231] Brown TD, Dalton PD, Hutmacher DW. Direct writing by way of melt electrospinning. Adv Mater 2011;23:5651-7.

[232] http://www.toray.com/technology/technology/core/cor_002.html

[233] Ellison CJ, Phatak A, Giles DW, Macosko CW, Bates FS. Melt blown nanofibers: Fiber diameter distributions and onset of fiber breakup. Polymer 2007;48:3306-16.

[234] Xin S, Wang X. Mechanism of fiber formation in melt blowing. Ind Eng Chem Res 2012;51:10621-8.

[235] Xie S, Zeng Y. Turbulent air flow field and fiber whipping motion in the melt blowing process: experimental study. Ind Eng Chem Res 2012;51:5346-52.

[236] Hassounah IA, Rowland WC, Sparks SA, Orler EB, Joseph EG, Camelio JA, Mahajan RL. Processing of multilayered filament composites by melt blown spinning. J Appl Polym Sci $2014 ; 131: 40786 / 1-8$

[237] Persano L, Camposeo A, Di Benedetto F, Stabile R, Laera AM, Piscopiello E, Tapfer L, Pisignano D. CdS-polymer nanocomposites and light-emitting fibers by in situ electron-beam synthesis and lithography. Adv Mater 2012;24:5320-6.

[238] Chen Y, Luo Y. Precisely defined heterogeneous conducting polymer nanowire arrays fabrication and chemical sensing applications. Adv Mater 2009;21:2040-4.

[239] Bong KW, Bong KT, Pregibon DC, Doyle PS. Hydrodynamic focusing lithography. Angew Chem Int Ed 2010;49:87-90.

[240] Laza SC, Polo M, Neves AAR, Cingolani R, Camposeo A, Pisignano D. Two-photon continuous flow lithography. Adv Mater 2012;24:1304-8.

[241] Wang P, Wang Y, Tong L. Functionalized polymer nanofibers: a versatile platform for manipulating light at the nanoscale. Light Sci Appl 2013;2:e102/1-10. 
Published in Progress in Polymer Science 43, 48-95, doi: 10.1016/j.progpolymsci.2014.10.001 (2015).

[242] Yu H, Li B. Wavelength-converted wave-guiding in dye-doped polymer nanofibers. Sci Rep $2013 ; 3: 1674 / 1-6$.

[243] Jung YS, Jung WC, Tuller HL, Ross CA. Nanowire conductive polymer gas sensor patterned using self-assembled block copolymer lithography. Nano Lett 2008;8:3776-80.

[244] O’Carroll DM, Petoukhoff CE, Kohl J, Yu B, Carter CM, Goodman S. Conjugated polymerbased photonic nanostructures. Polym Chem 2013;4:5181-96.

[245] Camposeo A, Persano L, Pisignano D. Light-emitting electrospun nanofibers for nanophotonics and optoelectronics. Macromol Mater Eng 2013;298:487-503.

[246] Tong L, Zi F, Guo X, Lou J. Optical microfibers and nanofibers: a tutorial. Opt Comm 2012;285:4641-7.

[247] Richard-Lacroix M, Pellerin C. Molecular orientation in electrospun fibers: from mats to single fibers. Macromolecules 2013;46:9473-93.

[248] Morello G, Polini A, Girardo S, Camposeo A, Pisignano D. Enhanced emission efficiency in electrospun polyfluorene copolymer fibers. Appl Phys Lett 2013;102:211911/1-3.

[249] Baghgar M, Pentzer E, Wise AJ, Labastide JA, Emrick T, Barnes MD. Cross-linked functionalized poly(3-hexylthiophene) nanofibers with tunable excitonic coupling. ACS Nano $2013 ; 7: 8917-23$.

[250] Wang Y, La A, Ding Y, Liu Y, Lei Y. Novel signal-amplifying fluorescent nanofibers for naked-eye-based ultrasensitive detection of buried explosives and explosive vapors. Adv Funct Mater 2012;22:3547-55.

[251] So F, Krummacher B, Mathai MK, Poplavskyy D, Choulis SA, Choong V-E. Recent progress in solution processable organic light emitting devices. J Appl Phys 2007;102:091101/1-21. [252] Kamtekar KT, Monkman AP, Bryce MR. Recent advances in white organic light-emitting materials and devices (WOLEDs). Adv Mater 2010;22:572-82.

[253] Zhong C, Duan C, Huang F, Wu H, Cao Y. Materials and devices toward fully solution processable organic light-emitting diodes. Chem Mater 2011;23,326-40. 
Published in Progress in Polymer Science 43, 48-95, doi: 10.1016/j.progpolymsci.2014.10.001 (2015).

[254] Muccini M. A bright future for organic field-effect transistors. Nat Mater 2006;5:605-13. [255] Yang H, Lightner CR, Dong L. Light-emitting coaxial nanofibers. ACS Nano 2012;6:622-8. [256] www.rti.org/NLITe

[257] Das AJ, Lafargue C, Lebental M, Zyss J, Narayan KS. Three-dimensional microlasers based on polymer fibers fabricated by electrospinning. Appl Phys Lett 2011;99:263303/1-3. [258] Ta VD, Chen R, Ma L, Ying YJ, Sun HD. Whispering gallery mode microlasers and refractive index sensing based on single polymer fiber. Laser Photon Rev 2013;7:133-9. [259] Persano L, Camposeo A, Del Carro P, Fasano V, Moffa M, Manco R, D’Agostino S, Pisignano D. Distributed feedback imprinted electrospun fiber lasers. Adv Mater 2014; DOI: 10.1002/adma.201401945.

[260] Gunes S, Neugebauer H, Sariciftci NS. Conjugated polymer-based organic solar cells. Chem Rev 2007;107:1324-38.

[261] Mayer AC, Scully SR, Hardin BE, Rowell MW, McGehee MD. Polymer-based solar cells. Mater Today 2007;10:28-33.

[262] Kroon R, Lenes M, Hummelen JC, Blom PWM, De Boer B. Small bandgap polymers for organic solar cells (Polymer material development in the last 5 years). Polym Rev 2008;48:531-82. [263] Kim K, Kim BH, Joo S-H, Park J-S, Joo J, Jin J-I. Photoconductivity of single-bilayer nanotubes consisting of poly( $p$-phenylenevinylene) (PPV) and carbonized-PPV layers. Adv Mater $2005 ; 17: 464-8$.

[264] Xin Y, Huang Z, Jiang Z, Li D, Peng L, Zhai J, Wang D. Photoresponse of a single poly(pphenylene vinylene)-CdSe bulk-heterojunction submicron fiber. Chem Commun 2010;46:2316-8. [265] Xin Y, Huang ZH, Peng L, Wang DJ. Photoelectric performance of poly(p-phenylene vinylene)/ $/ \mathrm{Fe}_{3} \mathrm{O}_{4}$ nanofiber array. J Appl Phys 2009;105:086106/1-3.

[266] Imahori H, Kitaura S, Kira A, Hayashi H, Nishi M, Hirao K, Isoda S, Tsujimoto M, Takano M, Zhe Z, Miyato Y, Noda K, Matsushige K, Stranius K, Tkachenko NV, Lemmetyinen H, Qin L, 
Published in Progress in Polymer Science 43, 48-95, doi: 10.1016/j.progpolymsci.2014.10.001 (2015).

Hurst SJ, Mirkin CA. A photoconductive, thiophene-fullerene double-cable polymer, nanorod device. J Phys Chem Lett 2012;3:478-81.

[267] Tu D, Pagliara S, Camposeo A, Persano L, Cingolani R, Pisignano D. Single light-emitting polymer nanofiber field-effect transistors. Nanoscale 2010;2:2217-22.

[268] Tu D, Pagliara S, Cingolani R, Pisignano D. An electrospun fiber phototransistor by the conjugated polymer poly[2-methoxy-5-(2'-ethylhexyloxy)-1,4-phenylene-vinylene]. Appl Phys Lett 2011;98:023307/1-3.

[269] Wu P-T, Xin H, Sunjoo Kim F, Ren G, Jenekhe SA. Regioregular poly(3-pentylthiophene): synthesis, self-assembly of nanowires, high-mobility field-effect transistors, and efficient photovoltaic cells. Macromolecules 2009;42:8817-26.

[270] Xin H, Sunjoo Kim F, Jenekhe SA. Highly efficient solar cells based on poly(3butylthiophene) nanowires. J Am Chem Soc 2008;130:5424-5.

[271] Xin H, Reid OG, Ren G, Sunjoo Kim F, Ginger DS, Jenekhe SA. Polymer nanowire/fullerene bulk heterojunction solar cells: how nanostructure determines photovoltaic properties. ACS Nano 2010;4:1861-72.

[272] Kim B-G, Kim M-S, Kim J. Ultrasonic-assisted nanodimensional self-assembly of poly-3hexylthiophene for organic photovoltaic cells. ACS Nano 2010;4:2160-6.

[273] Kim T, Yang SJ, Kim SK, Choi HS, Park CR. Preparation of PCDTBT nanofibers with a diameter of $20 \mathrm{~nm}$ and their application to air-processed organic solar cells. Nanoscale $2014 ; 6: 2847-54$

[274] Seidler N, Lazzerini GM, Li Destri G, Marletta G, Cacialli F. Enhanced crystallinity and film retention of P3HT thin films for efficient organic solar cells by use of preformed nanofibers in solution. J Mater Chem C 2013;1:7748-57.

[275] Yang X, Loos J, Veenstra SC, Verhees WJH, Wienk MM, Kroon JM, Michels MAJ, Janssen RAJ. Nanoscale morphology of high-performance polymer solar cells. Nano Lett 2005;5:579-83. 
Published in Progress in Polymer Science 43, 48-95, doi: 10.1016/j.progpolymsci.2014.10.001 (2015).

[276] Xin H, Ren G, Sunjoo Kim F, Jenekhe SA. Bulk heterojunction solar cells from poly(3butylthiophene)/fullerene blends: in situ self-assembly of nanowires, morphology, charge transport, and photovoltaic properties. Chem Mater 2008;20:6199-207.

[277] Lee TH, Do K, Lee YW, Geon SS, Kim C, Ko J, Im SS. High-performance dye-sensitized solar cells based on PEDOT nanofibers as an efficient catalytic counter electrode. J Mater Chem 2012;22:21624-9.

[278] Peng S, Zhu P, Wu Y, Mhaisalkar SG, Ramakrishna S. Electrospun conductive polyanilinepolylactic acid composite nanofibers as counter electrodes for rigid and flexible dye-sensitized solar cells. RSC Adv 2012;2:652-7.

[279] Sundarrajan S, Murugan R, Nair AS, Ramakrishna S. Fabrication of P3HT/PCBM solar cloth by electrospinning technique. Mater Lett 2010;64:2369-72.

[280] Ding G, Wu Y, Weng Y, Zhang W, Hu Z. Solvent-assistant room temperature nanoimprinting-induced molecular orientation in poly(3-hexylthiophene) nanopillars. Macromolecules 2013;46:8638-43.

[281] Aryal M, Trivedi K, Hu W. Nano-confinement induced chain alignment in ordered P3HT nanostructures defined by nanoimprint lithography. ACS Nano 2009;3:3085-90.

[282] Hlaing H, Lu X, Hofmann T, Yager KG, Black CT, Ocko BM. Nanoimprint-induced molecular orientation in semiconducting polymer nanostructures. ACS Nano 2011;5:7532-8. [283] Gentili D, Sonar P, Liscio F, Cramer T, Ferlauto L, Leonardi F, Milita S, Dodabalapur A, Cavallini M. Logic-gate devices based on printed polymer semiconducting nanostripes. Nano Lett $2013 ; 13: 3643-7$.

[284] Duvail JL, Long Y, Cuenot S, Chen A, Gu C. Tuning electrical properties of conjugated polymer nanowires with the diameter. Appl Phys Lett 2007;90:102114/1-3.

[285] Pinto NJ, González R, Johnson AT, MacDiarmid AG. Electrospun hybrid organic/inorganic semiconductor Schottky nanodiode. Appl Phys Lett 2006;89:033505/1-3. 
Published in Progress in Polymer Science 43, 48-95, doi: 10.1016/j.progpolymsci.2014.10.001 (2015).

[286] Martínez O, Bravo AG, Pinto NJ. Fabrication of Poly(vinylidene fluoride-

trifluoroethylene)/poly(3,4-ethylenedioxythiophene)-polystyrene sulfonate composite nanofibers via electrospinning. Macromolecules 2009;42:7924-9.

[287] Pinto NJ, Ramosa I, Rojas R, Wang P-C andJohnson AT. Electric response of isolated electrospun polyaniline nanofibers to vapors of aliphatic alcohols. Sens Actuators B Chem 2008;129:621-7.

[288] Liu H, Kameoka J, Czaplewski DA, Craighead HG. Polymeric nanowire chemical sensor. Nano Lett 2004;4:671-5.

[289] Wang Q, Dong X, Pang Z, Du Y, Xia X, Wei Q, Huang F. Ammonia sensing behaviors of $\mathrm{TiO}_{2}$-PANI/PA6 composite nanofibers. Sensors 2012;12:17046-57.

[290] Zhijiang C, Ruihan Z, Xingjaun S. Preparation and characterization of polyindole nanofibers by electrospinning method. Synth Met 2012;162:2069-74.

[291] Long Y, Chen Z, Shen J, Zhang Z, Zhang L, Huang K, Wan M, Jin A, Gu C, Duvail JL. Magnetoresistance studies of polymer nanotube/wire pellets and single polymer nanotubes/wires. Nanotechnology 2006;17:5903-11.

[292] Nath C, Kumar A. Effect of temperature and magnetic field on the electrical transport of polyaniline nanofibers. J Appl Phys 2013;113:093707/1-10.

[293] Pinto NJ, Johnson AT, MacDiarmid AG, Mueller CH, Theofylaktos N, Robinson DC, Miranda FA. Electrospun polyaniline/polyethylene oxide nanofiber field-effect transistor. Appl Phys Lett 2003;83:4244-6.

[294] Tran HD, Wang Y, D’Arcy JM, Kaner RB. Toward an understanding of the formation of conducting polymer nanofibers. ACS Nano 2008;2:1841-8.

[295] Liu H, Reccius CH, Craighead HG. Single electrospun regioregular poly(3-hexylthiophene) nanofiber field-effect transistor. Appl Phys Lett 2005;87:253106/1-3. 
Published in Progress in Polymer Science 43, 48-95, doi: 10.1016/j.progpolymsci.2014.10.001 (2015).

[296] Chou C-C, Wu H-C, Lin C-J, Ghelichkhani E, Chen W-C. Morphology and field-effect transistor characteristics of electrospun nanofi bers prepared from crystalline poly(3hexylthiophene) and polyacrylate blends. Macromol Chem Phys 2013;214:751-60. [297] Oosterbann WD, Bolsée J-C, Gadisa A, Vrindts V, Bertho S, D’Haen J, Cleij TJ, Lutsen L, McNeill CR, Thomsen L, Manca JV, Vanderzande D. Alkyl-chain-length-independent hole mobility via morphological control with poly(3-alkylthiophene) nanofibers. Adv Funct Mater 2010;20:792-802.

[298] Kim JH, Lee DH, Yang DS, Heo DU, Kim KH, Shin J, Kim H-J, Baek K-Y, Lee K, Baik H, Cho MJ, Choi DH. Novel polymer nanowire crystals of diketopyrrolopyrrole-based copolymer with excellent charge transport properties. Adv Mater 2013;25:4102-6.

[299] Chang H-S, Liu C-L, Chen W-C. Flexible nonvolatile transistor memory devices based on one-dimensional electrospun P3HT:Au hybrid nanofibers. Adv Funct Mater 2013;23:4960-8. [300] Hsu BBY, Seifter J, Takacs CJ, Zhong C, Tseng H-R, Samuel IDW, Namdas EB, Bazan GC, Huang F, Cao Y, Heeger AJ. Ordered polymer nanofibers enhance output brightness in bilayer light-emitting field-effect transistors. ACS Nano 2013;7:2344-51.

[301] Zhao Z, Li J, Yuan X, Li X, Zhang Y, Sheng J. Preparation and properties of electrospun poly(vinylidene fluoride) membranes. J Appl Polym Sci 2005;97:466-74.

[302] Yee WA, Kotaki M, Liu Y, Lu X. Morphology, polymorphism behavior and molecular orientation of electrospun poly(vinylidene fluoride) fibers. Polymer 2007;48:512-21.

[303] Zheng J, He A, Li J, Han CC. Polymorphism control of poly(vinylidene fluoride) through electrospinning. Macromol Rapid Commun 2007;28:2159-62.

[304] Choi SW, Kim JR, Ahn YR, Jo SM, Cairns EJ. Characterization of electrospun PVdF fiberbased polymer electrolytes. Chem Mater 2007;19:104-15.

[305] Andrew JS, Clarke DR. Effect of electrospinning on the ferroelectric phase content of polyvinylidene difluoride fibers. Langmuir 2008;24:670-2. 
Published in Progress in Polymer Science 43, 48-95, doi: 10.1016/j.progpolymsci.2014.10.001 (2015).

[306] Andrew JS, Clarke DR. Enhanced ferroelectric phase content of polyvinylidene difluoride fibers with the addition of magnetic nanoparticles. Langmuir 2008;24:8435-8.

[307] Huang S, Yee WA, Tjiu WC, Liu Y, Kotaki M, Boey YCF, Ma J, Liu T, Lu X.

Electrospinning of polyvinylidene difluoride with carbon nanotubes: synergistic effects of extensional force and interfacial interaction on crystalline structures. Langmuir 2008;24:13621-6. [308] Hansen BJ, Liu Y, Yang R, Wang ZL. Hybrid nanogenerator for concurrently harvesting biomechanical and biochemical energy. ACS Nano 2010;4:3647-52.

[309] Pu J, Yan X, Jiang Y, Chang C, Lin L. Piezoelectric actuation of direct-write electrospun fibers. Sens Actuators A Phys 2010;164:131-6.

[310] Fang J, Wang X, Lin T. Electrical power generator from randomly oriented electrospun poly(vinylidene fluoride) nanofibre membranes. J Mater Chem 2011;21:11088-91.

[311] Mandal D, Yoon S, Kim KJ. Origin of piezoelectricity in an electrospun poly(vinylidene fluoride-trifluoroethylene) nanofiber web-based nanogenerator and nano-pressure sensor. Macromol Rapid Commun 2011;32:831-7.

[312] He F, Sarkar M, Lau S, Fan J, Chan LH. Preparation and characterization of porous poly(vinylidene fluoride-trifluoroethylene) copolymer membranes via electrospinning and further hot pressing. Polym Test 2011;30:436-41.

[313] Baji A, Mai Y-W, Lib Q, Liu Y. Electrospinning induced ferroelectricity in poly(vinylidene fluoride) fibers. Nanoscale 2011;3:3068-71.

[314] Wang YR, Zheng JM, Ren GY, Zhang PH, Xu C. A flexible piezoelectric force sensor based on PVDF fabrics. Smart Mater Struct 2011;20:045009/1-7.

[315] Dhakras D, Borkar V, Ogale S, Jog J. Enhanced piezoresponse of electrospun PVDF mats with a touch of nickel chloride hexahydrate salt. Nanoscale 2012;4:752-6.

[316] Fang J, Niu H, Wang H, Wang X, Lin T. Enhanced mechanical energy harvesting using needleless electrospun poly(vinylidene fluoride) nanofibre webs. Energy Environ Sci 2013;6:2196202. 
Published in Progress in Polymer Science 43, 48-95, doi: 10.1016/j.progpolymsci.2014.10.001 (2015).

[317] Edmondson D, Jana S, Wood D, Fang C, Zhang M. Uniaxially-aligned PVDF nanofibers as a sensor and transmitter for biotelemetry. Analyst 2013;138:7135-9.

[318] Soin N, Shah TH, Anand SC, Geng J, Pornwannachai W, Mandal P, Reid D, Sharma S, Hadimani RL, Vatansever Bayramol D, Siores E. Novel “3-D spacer” all fibre piezoelectric textiles for energy harvesting applications. Energy Environ Sci 2014; 7:1670-1679.

[319] Shaohui L, Jiwei Z, Jinwen W, Shuangxi X, Wenqin Z. Enhanced energy storage density in poly(vinylidene fluoride) nanocomposites by a small loading of suface-hydroxylated $\mathrm{Ba}_{0.6} \mathrm{Sr}_{0.4} \mathrm{TiO}_{3}$ nanofibers. ACS Appl Mater Interfaces 2014;6:1533-40.

[320] Farrar D, Ren K, Cheng D, Kim S, Moon W, Wilson WL, West JE, Yu SM. Permanent polarity and piezoelectricity of electrospun $\alpha$-helical poly( $\alpha$-amino acid) fibers. Adv Mater 2011;23:3954-8.

[321] Dubey N, Leclerc M. Conducting polymers: efficient thermoelectric materials. J Polym Sci Part B Polym Phys 2011;49:467-75.

[322] Bubnova O, Khan ZU, Wang H, Braun S, Evans DR, Fabretto M, Hojati-Talemi P, Dagnelund D, Arlin J-B, Geerts YH, Desbief S, Breiby DW, Andreasen JW, Lazzaroni R, Chen WM, Zozoulenko I, Fahlman M, Murphy PJ, Berggren M, Crispin X. Semi-metallic polymers. Nat Mater 2014;13:190-4.

[323] Chabinyc M. Behind organics’ thermopower. Nat Mater 2014;13:119-21.

[324] Sun Y, Wei Z, Xu W, Zhu D. A three-in-one improvement in thermoelectric properties of polyaniline brought by nanostructures. Synth Met 2010;160:2371-6.

[325] Taggart DK, Yang Y, Kung S-C, McIntire TM, Penner RM. Enhanced thermoelectric metrics in ultra-long electrodeposited PEDOT nanowires. Nano Lett 2011;11:125-31.

[326] Wang Q, Yao Q, Chang J, Chen L. Enhanced thermoelectric properties of CNT/PANI composite nanofibers by highly orienting the arrangement of polymer chains. J Mater Chem 2012;22:17612-8. 
Published in Progress in Polymer Science 43, 48-95, doi: 10.1016/j.progpolymsci.2014.10.001 (2015).

[327] Otero TF, Martinez JG, Arias-Pardilla J. Biomimetic electrochemistry from conducting polymers. A review. Artificial muscles, smart membranes, smart drug delivery and computer/neuron interfaces. Electrochim Acta 2012;84:112-28.

[328] Gong T, Li W, Chen H, Wang L, Shao S, Zhou S. Remotely actuated shape memory effect of electrospun composite nanofibers. Acta Biomater 2012;8:1248-59.

[329] Bassil M, Ibrahim M, El Tahchi M. Artificial muscular microfibers: hydrogel with high speed tunable Electroactivity. Soft Matter 2011;7:4833-8.

[330] Samatham R, Park I-S, Kim KJ, Nam J-D, Whisman N, Adams J. Electrospun nanoscale polyacrylonitrile artificial muscle. Smart Mater Struct 2006;15:N152-6.

[331] Gestos A, Whitten PG, Wallace GG, Spinks GM. Actuating individual electrospun hydrogel nanofibres. Soft Matter 2012;8:8082-7.

[332] Lee DY, Kim Y, Lee S-J, Lee M-H, Lee J-Y, Kim B-Y, Cho N-I. Characteristics of chemomechanically driven polyacrylonitrile fiber gel actuators. Mat Sci Eng C-Bio S 2008;28:294-8. [333] Nakagawa H, Hara Y, Maeda S, Hasimoto S. A pendulum-like motion of nanofiber gel actuator synchronized with external periodic pH oscillation. Polymer 2011;3:405-12. [334] Baker CO, Shedd B, Innis PC, Whitten PG, Spinks GM, Wallace GG and Kaner RB. Monolithic actuators from flash-welded polyaniline nanofibers. Adv Mater 2008;20:155-8. [335] Gao H, Zhang J, Yu W, Li Y, Zhu S, Li Y, Wang T, Yang B. Monolithic polyaniline/polyvinyl alcohol nanocomposite actuators with tunable stimuli-responsive properties. Sens Actuators B Chem 2010;145:839-46.

[336] Ismail YA, Shin SR, Shin KM, Yoon SG, Shon K, Kim SI, Kim SJ. Electrochemical actuation in chitosan/polyaniline microfibers for artificial muscles fabricated using an in situ polymerization. Sens Actuators B Chem 2008;129:834-40.

[337] Ismail YA, Martínez JG, Al Harrasi AS, Kim SJ, Otero TF. Sensing characteristics of a conducting polymer/hydrogel hybrid microfiber artificial muscle. Sens Actuators B Chem 2011;160:1180-90. 
Published in Progress in Polymer Science 43, 48-95, doi: 10.1016/j.progpolymsci.2014.10.001 (2015).

[338] Ohm C, Morys M, Forst FR, Braun L, Eremin A, Serra C, Stannarius R, Zentel R. Preparation of actuating fibres of oriented main-chain liquid crystalline elastomers by a wetspinning process. Soft Matter 2011;7:3730-4.

[339] Ismail YA, Shin MK, Kim SJ. A nanofibrous hydrogel templated electrochemical actuator: from single mat to a rolled-up structure. Sensor Actuat B Chem 2009;136:438-43. [340] McKeon-Fischer KD, Flagg DH, Freeman JW. Poly(acrylic acid)/poly(vinyl alcohol) compositions coaxially electrospun with poly( $\varepsilon$-caprolactone) and multi-walled carbon nanotubes to create nanoactuating scaffolds. Polymer 2011;52:4736-43.

[341] McDowell JJ, Zacharia NS, Puzzo D, Manners I, Ozin GA. Electroactuation of alkoxysilanefunctionalized polyferrocenylsilane microfibers. J Am Chem Soc 2010;132:3236-7.

[342] Barhate RS, Ramakrishna S. Nanofibrous filtering media: filtration problems and solutions from tiny materials. J Membr Sci 2007;296:1-8.

[343] Lim SH, Mao H-Q. Electrospun scaffolds for stem cell engineering. Adv Drug Deliv Rev 2009;61:1084-96.

[344] Zahedi P, Rezaeian I, Ranaei-Siadat S-O, Jafari S-H, Supaphol P. A review on wound dressings with an emphasis on electrospun nanofibrous polymeric bandages. Polym Adv Technol 2010;21:77-95. 
Published in Progress in Polymer Science 43, 48-95, doi: 10.1016/j.progpolymsci.2014.10.001 (2015).

Tables

\begin{tabular}{|c|c|c|c|c|c|c|c|}
\hline & $\begin{array}{c}\text { Self- } \\
\text { assembly }\end{array}$ & $\begin{array}{l}\text { Templates } \\
\text { (hard) }\end{array}$ & $\begin{array}{c}\text { Templates } \\
\text { (soft) }\end{array}$ & $\begin{array}{l}\text { Electro- } \\
\text { spinning }\end{array}$ & $\begin{array}{c}\text { Melt / } \\
\text { Dry } \\
\text { spinning }\end{array}$ & $\begin{array}{c}\text { Drawing / } \\
\text { Lithography }\end{array}$ & $\begin{array}{l}\text { Polymerization } \\
\text { / Interfacial }\end{array}$ \\
\hline BBL & 144 & & & & & & \\
\hline F8T2 & & 52 & & & & & \\
\hline MEH-PPV & & 8 & & $9-20$ & & 21 & \\
\hline P3HT- $b$-PDMS & 149 & & & & & & \\
\hline PAN & & & $\begin{array}{l}156,169 \\
171\end{array}$ & 208 & & & $\begin{array}{l}51,124,125 \\
127,128,130\end{array}$ \\
\hline PEDOT & & $182-184$ & $\begin{array}{l}112,152 \\
155,158- \\
160\end{array}$ & & & 238 & \\
\hline $\begin{array}{l}\text { Poly[2,5-(2'- } \\
\text { ethylhexyloxy)]-1,4- } \\
\text { phenylenevinylene }\end{array}$ & & & & 22 & & & \\
\hline $\begin{array}{l}\text { Poly[1- } \\
\text { (trimethylsilyl)phenyl-2- } \\
\text { phenylacetylene] }\end{array}$ & & & & 92 & & & \\
\hline $\begin{array}{l}\text { Poly[3,4-(4,5- } \\
\text { didodecylphenylene) } \\
\text { dioxythiophene }]\end{array}$ & 136 & & & & & & \\
\hline PFO & & $53-59$ & & 9,60 & & & \\
\hline F8BT & & & & 14,61 & & 21,62 & \\
\hline $\begin{array}{l}\text { Poly[(9,9-dioctylfluorenyl- } \\
\text { 2,7-diyl)-co-(1,4- } \\
\text { diphenylenevinylene-2- } \\
\text { methoxy-5-\{2 } \\
\text { ethylhexyloxy\}-benzene)] }\end{array}$ & & & & 14,91 & & 21 & \\
\hline $\begin{array}{l}\text { Poly[(9,9-dioctylfluorenyl- } \\
\text { 2,7-diyl)-alt-co-(9-hexyl- } \\
\text { 3,6-carbazole)] }\end{array}$ & & & & 14 & & & \\
\hline Polyacetylene & & & $64-66$ & & & & \\
\hline $\begin{array}{l}\text { Poly (cyclopenta } \\
\text { dithiophene- } \\
\text { benzothiadiazole) }\end{array}$ & 138 & & & & & & \\
\hline $\begin{array}{l}\text { Polythiophenes (including } \\
\text { P3ATs) }\end{array}$ & $\begin{array}{c}32-41 \\
137\end{array}$ & 42,43 & 44 & $\begin{array}{c}9,12,20 \\
45-49 \\
209\end{array}$ & & 50 & 51 \\
\hline PPV & & $23-25$ & & $26-28$ & 29 & 30 & \\
\hline PPY & & 180,181 & $\begin{array}{l}150,153 \\
170,171\end{array}$ & & & 238 & \\
\hline PVK & & 95,179 & & 10 & & & \\
\hline
\end{tabular}

Table 1. Summary of the main conjugated polymers which have been processed to synthetize nanofibers, and corresponding fabrication technologies. 

(2015).

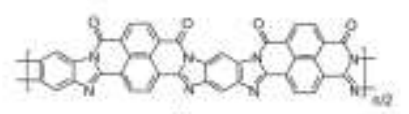

a

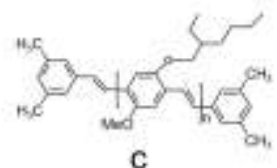

C

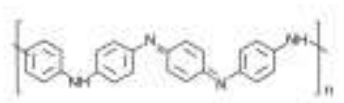

e
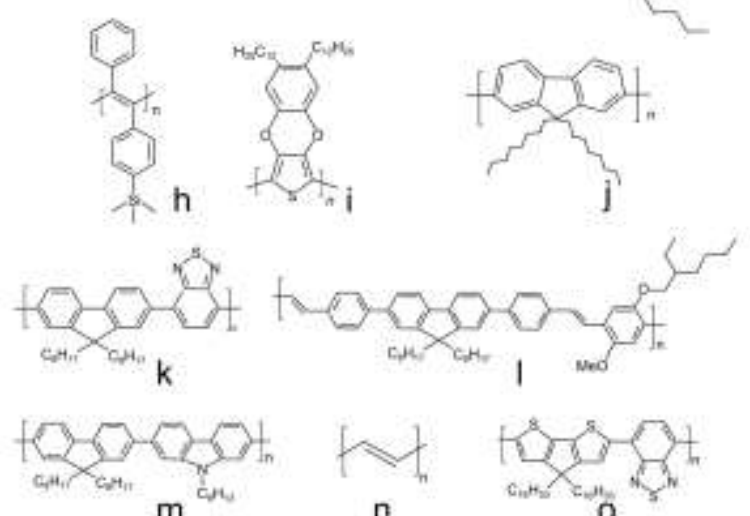

$\mathrm{m}$

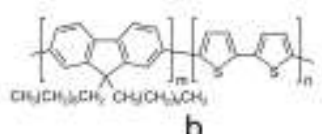

b
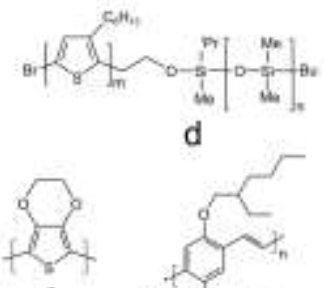

$\mathrm{f}$
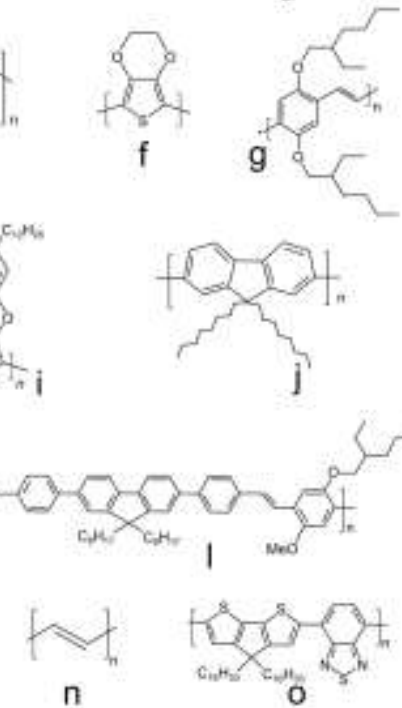

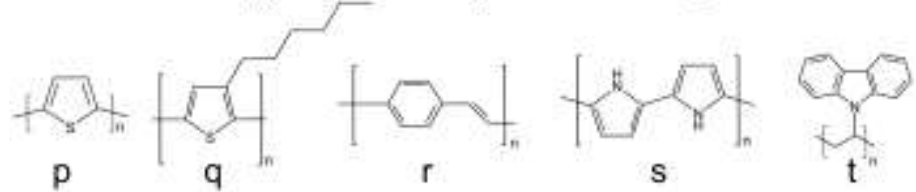

Fig. 1 Molecular structures of the main conjugated polymers from which nanofibers have been produced. BBL (a) [144], F8T2 (b), MEH-PPV (c), P3HT-b-PDMS (d) [149], PAN (emeraldine base, e), PEDOT (f), poly[2,5-(2'ethylhexyloxy)]-1,4-phenylenevinylene (g), poly[1-(trimethylsilyl)phenyl-2-phenylacetylene] (h) [92], poly[3,4-(4,5didodecylphenylene) dioxythiophene] (i) [136], PFO (j), F8BT (k), poly[(9,9-dioctylfluorenyl-2,7-diyl)-co-(1,4diphenylenevinylene-2-methoxy-5-\{2 ethylhexyloxy $\}$-benzene) $]$ (1), poly[(9,9-dioctylfluorenyl-2,7-diyl)-alt-co-(9hexyl-3,6-carbazole)] (m), trans-polyacetylene (n), poly (cyclopenta dithiophene-benzothiadiazole) (o) [138], polythiophene (p), P3HT (q), PPV (r), PPY (s), and PVK (t). Relevant related references are also listed in Table 1. 

(2015).

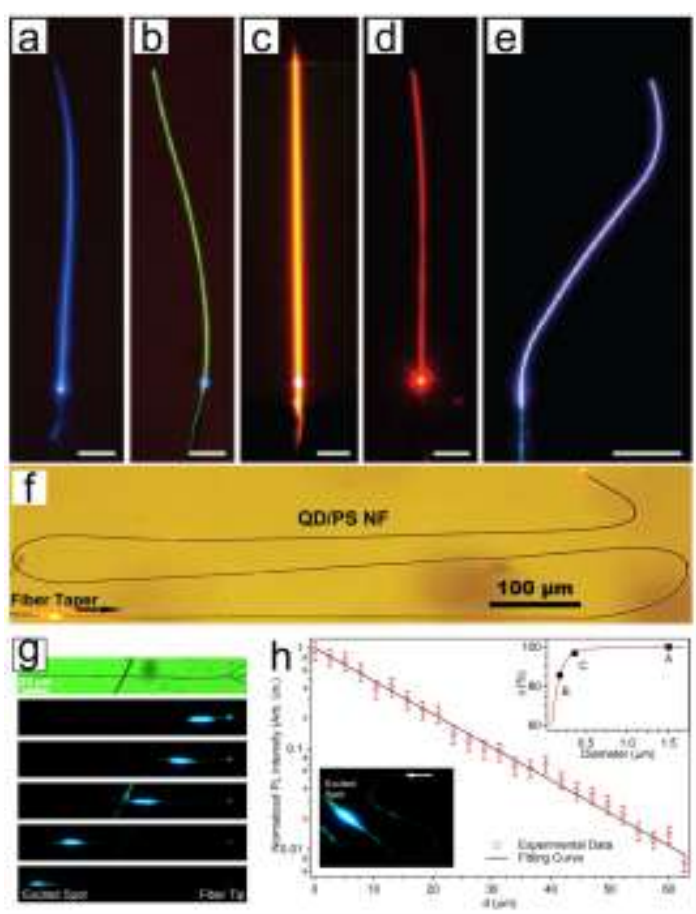

Fig. 2 (a-e) Fluorescence micrographs of drawn nanofibers emitting at various wavelengths. Fibers are made of perylene-doped polystyrene (a), polyacrylamide embedding fluorescein sodium salt-doped (b) and tris(2,2'bipyridine)ruthenium(II) chloride (c), zinc phthalocyanine-doped PEO (d), and polystyrene embedding perylene, rhodamine B and zinc phthalocyanine (e). Scale bars $=50 \mu \mathrm{m}$. Adapted with permission from ACS Nano 2010;4:53328 [84]. Copyright (c) 2010 American Chemical Society. (f) Optical micrograph of a polystyrene fiber doped with $\mathrm{CdSe} / \mathrm{ZnS}$ core-shell quantum dots (here abbreviated as QD/PS NF) and having diameter of $560 \mathrm{~nm}$. Light at a wavelength of $672 \mathrm{~nm}$ is launched by a fiber taper (bottom-left part of the figure) and travels across the fiber up to the other tip (top-right). Adapted with permission from Adv Mater 2011;23:3770-4 [83]. Copyright (c) 2011 Wiley-VCH Verlag GmbH \& Co. KGaA. (g, h) Bright field optical micrograph (top panel in g), and fluorescence micrographs of an electrospun poly[(9,9-dioctylfluorenyl-2,7-diyl)-co-( $N, N^{\prime}$-diphenyl)- $N, N^{\prime}$-di(p-butyl-oxy-phenyl)-1,4-diaminobenzene)] nanofiber excited by a focused laser beam at variable distances, $d$, from the fiber tip (g), and corresponding light intensity vs. $d$ data (h). The light waveguided and then exiting the tip is clearly visible in the micrographs. The continuous line in (h) is the best fit of data by an exponential decay function, from which the optical loss coefficient can be calculated. The bottom-left inset in (h) shows a bent fiber, whereas the plot in the top-right inset displays the calculated fraction of guided power in the fundamental mode vs. the fiber diameter. The points A, B and C are obtained by using different solvent mixtures to electrospin the corresponding fibers. Adapted with permission from Macromolecules 2013;46:5935-42 [86]. Copyright @ 2013 American Chemical Society. 
Published in Progress in Polymer Science 43, 48-95, doi: 10.1016/j.progpolymsci.2014.10.001 (2015).
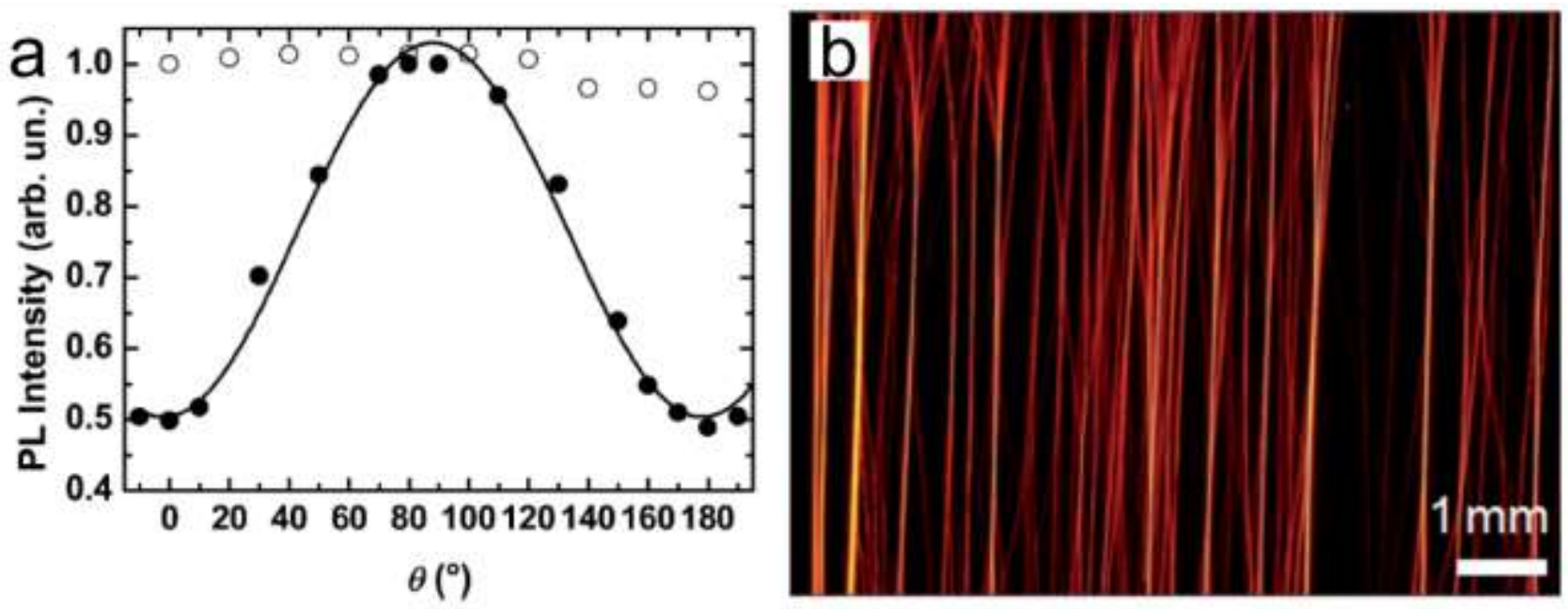

Fig. 3 (a) Dependence of the emission collected from an uniaxially aligned array of conjugated polymer nanofibers realized by nanofluidic lithography, on the angle, $\theta$, defined by the fiber longitudinal axis and the axis of a polarizer. Data from fibers are displayed as full circles, whereas empty circles indicate data from reference films, which do not show modulation related to polarized emission. Data from fibers are instead well described by a Malus' law, i.e. intensity $\cong \cos ^{2}(\theta)$, which evidences the preferential polarization of emitted light along the fiber length. Adapted with permission from Adv Mater 2008;20:4158-62 [21]. Copyright (c) 2008 Wiley-VCH Verlag GmbH \& Co. KGaA. (b) Fluorescence micrograph of an uniaxially aligned array of MEH-PPV/PEO electrospun nanofibers. Adapted with permission from J Mater Chem 2012;22:4695-703 [17]. Copyright @ 2012 The Royal Society of Chemistry. 
Published in Progress in Polymer Science 43, 48-95, doi: 10.1016/j.progpolymsci.2014.10.001 (2015).
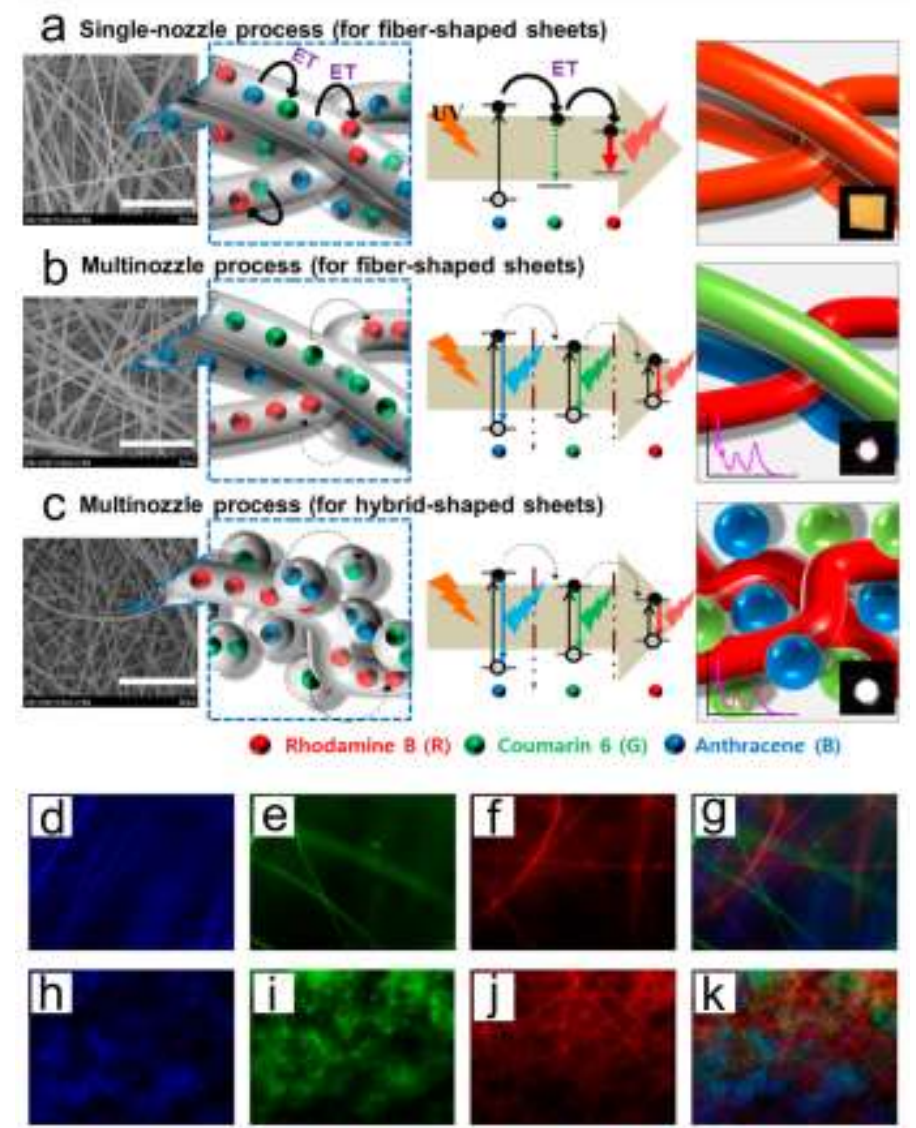

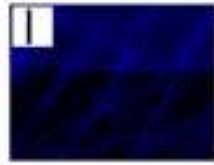

blue channel

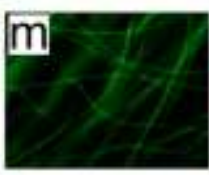

green channel

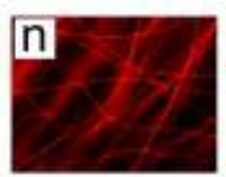

red channel

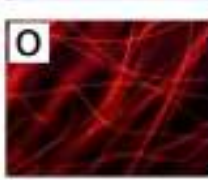

merged

Fig. 4 Schematics of production of light-emitting electrospun sheets by a single-nozzle (a) or by multi-nozzle techniques (b and c), and fluorescence micrographs of sheets made by the (b) method (d-g), by the (c) method (h-k), and by the (a) method (1-o). In (a), different dyes are embedded in the same nanofiber, thus promoting energy transfer (here ET) phenomena from high-energy donors to low-energy acceptors. In (b), fibers produced by different nozzles are doped with different dyes, reducing ET phenomena considerably. In (c), beads are produced by two types of nozzles, and fibers by the other type. Also in this case, different dopants are used for the different species of particles. The bottom-right insets of (a-c) show the corresponding sheets under photo-excitation. The overall emission is reddish (a) or white (b, c), respectively. In (d-o), different panels correspond to sheets realized with different relative concentrations of the involved dyes and particles. A wide tuneability is achieved in the visible range. Adapted with permission from ACS Appl Mater Interfaces 2013;5:6038-44 [98]. Copyright @ 2013 American Chemical Society. 
Published in Progress in Polymer Science 43, 48-95, doi: 10.1016/j.progpolymsci.2014.10.001 (2015).
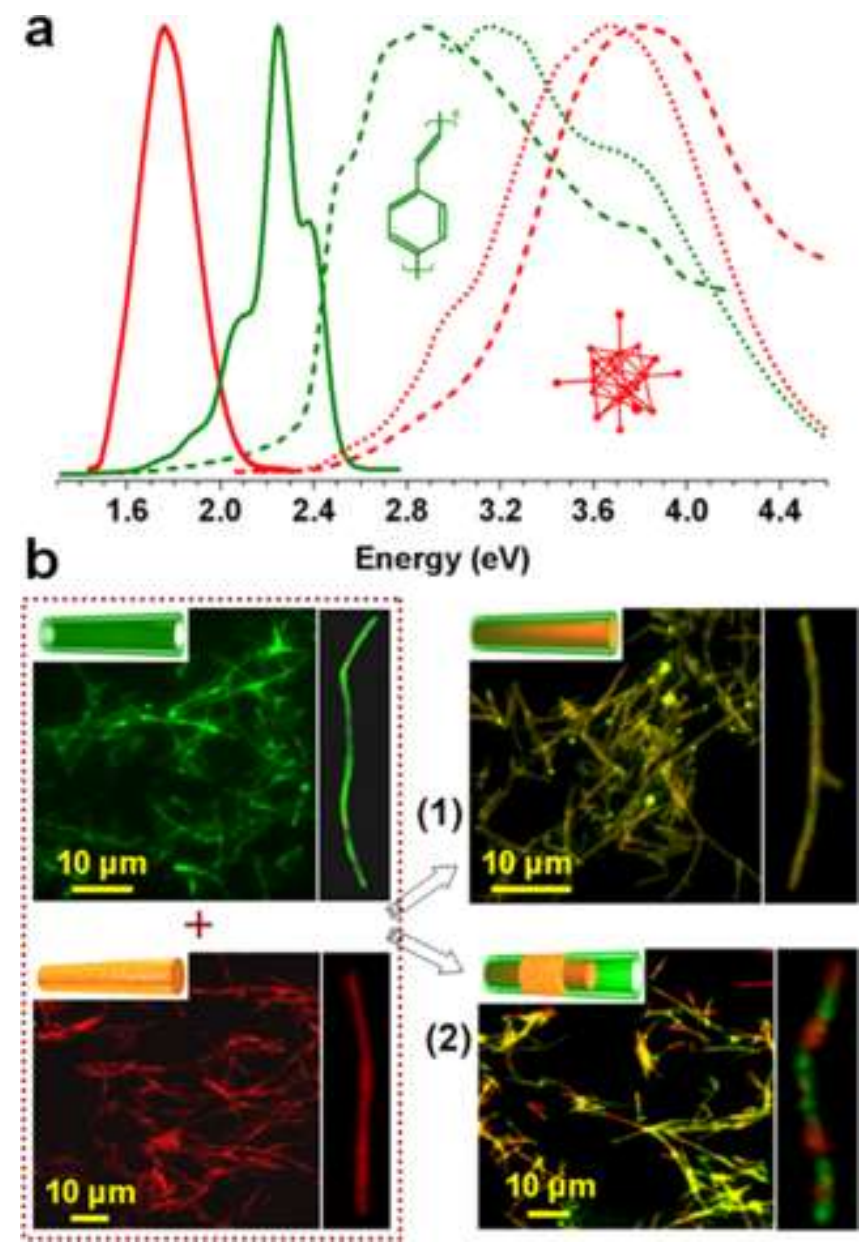

Fig. 5 (a) Normalized absorption (dashed lines), excitation (dotted lines) and luminescence (solid lines) spectra of films of PPV, emitting in the green region of the visible spectrum, and of $\left(n-\mathrm{Bu}_{4} \mathrm{~N}\right)_{2}\left[\mathrm{Mo}_{6} \mathrm{Br}_{8} \mathrm{~F}_{6}\right]$, which is a phosphorescent red emitter, together with the corresponding molecular structures, and (b) fluorescent micrographs of PPV nanotubes (top-left), of $\left(n-\mathrm{Bu}_{4} \mathrm{~N}\right)_{2}\left[\mathrm{Mo}_{6} \mathrm{Br}_{8} \mathrm{~F}_{6}\right] / \mathrm{PMMA}$ nanowires (bottom-left), and of coaxial nanowires with a $(n$ $\left.\mathrm{Bu}_{4} \mathrm{~N}\right)_{2}\left[\mathrm{Mo}_{6} \mathrm{Br}_{8} \mathrm{~F}_{6}\right] / \mathrm{PMMA}$ core and a continuous (1, top-right) and a discontinuous PPV shell (2, bottom-right), respectively. Reproduced with permission from ACS Nano 2013;7:2977-87 [108]. Copyright (C) 2013 American Chemical Society. 

(2015).

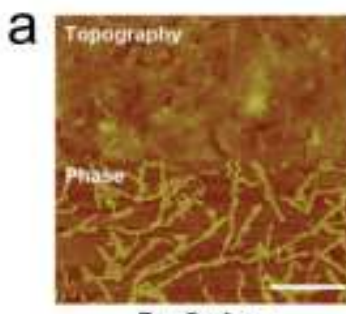

Top Surface

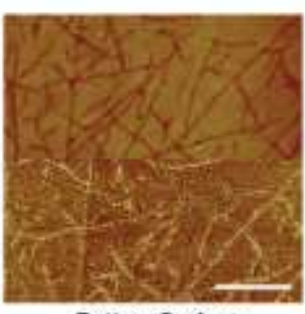

Bottom Surface

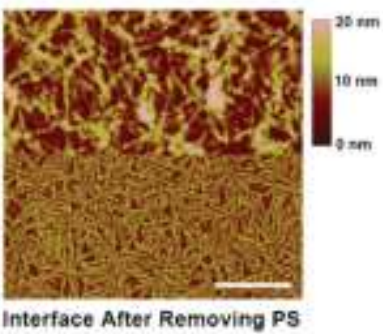

Interface After Removing PS
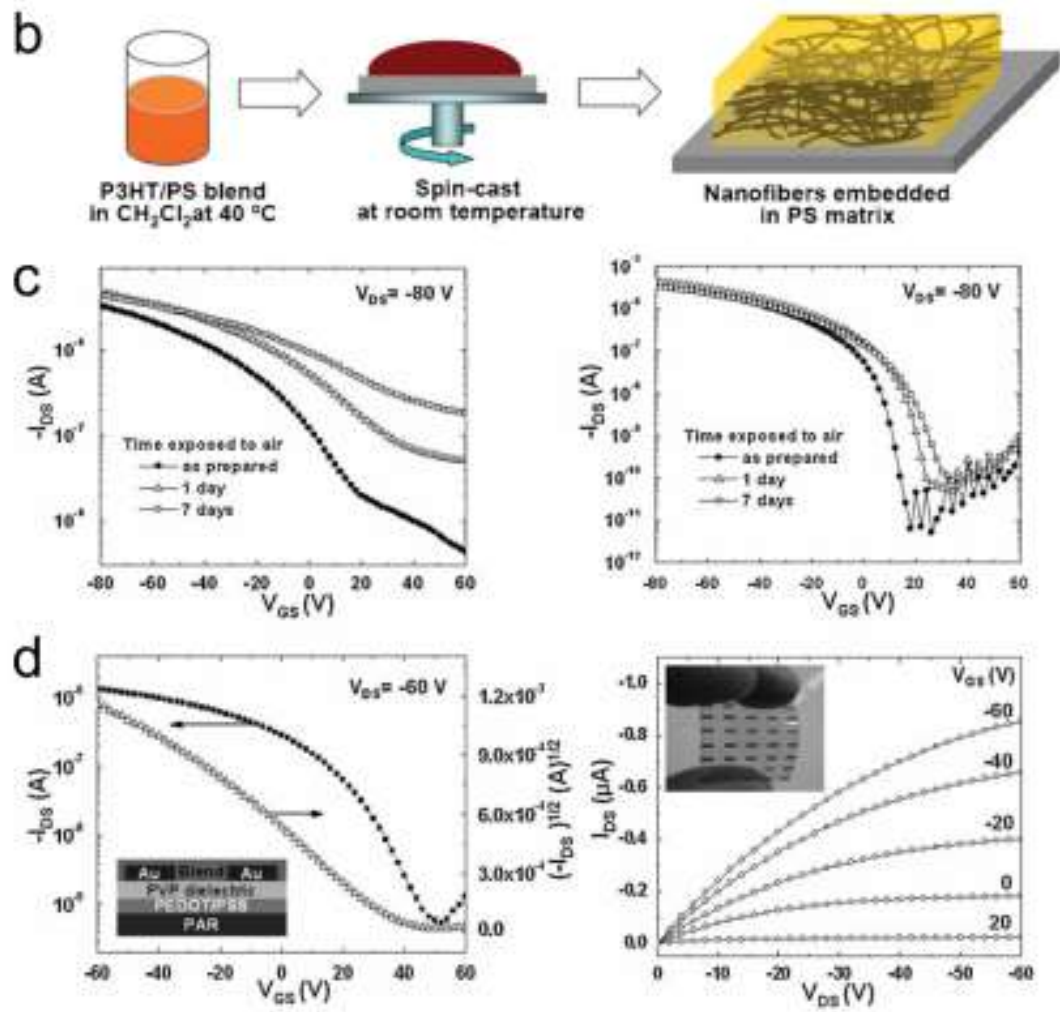

Fig. 6 P3HT/polystyrene with formation of conductive nanofibers, and devices made thereof. (a) Atomic force microscopy (AFM) topographic images and phase images of a P3HT/polystyrene film realized by spin coating from a dichloromethane solution. The top and the bottom sample surfaces are shown, together with the interface, imaged upon dissolving the polystyrene matrix. Scale bar $=500 \mathrm{~nm}$. (b) Process schematics. (c) Transfer characteristics showing the behavior of the drain-source current (here, $\left.I_{D S}\right) v s$. gate-source voltage (here, $V_{G S}$ ) and its dependence on air exposure for FETs based on P3HT (left) and on P3HT/polystyrene with fibril formation (right). (d) Transfer (left) and inputoutput (right) characteristics of flexible FETs based on P3HT/polystyrene. The device scheme and photograph are displayed in the insets of the left and of the right plots, respectively. Adapted with permission from Adv Mater 2009;21:1349-53 [110]. Copyright @ 2009 Wiley-VCH Verlag GmbH \& Co. KGaA. 
Published in Progress in Polymer Science 43, 48-95, doi: 10.1016/j.progpolymsci.2014.10.001 (2015).
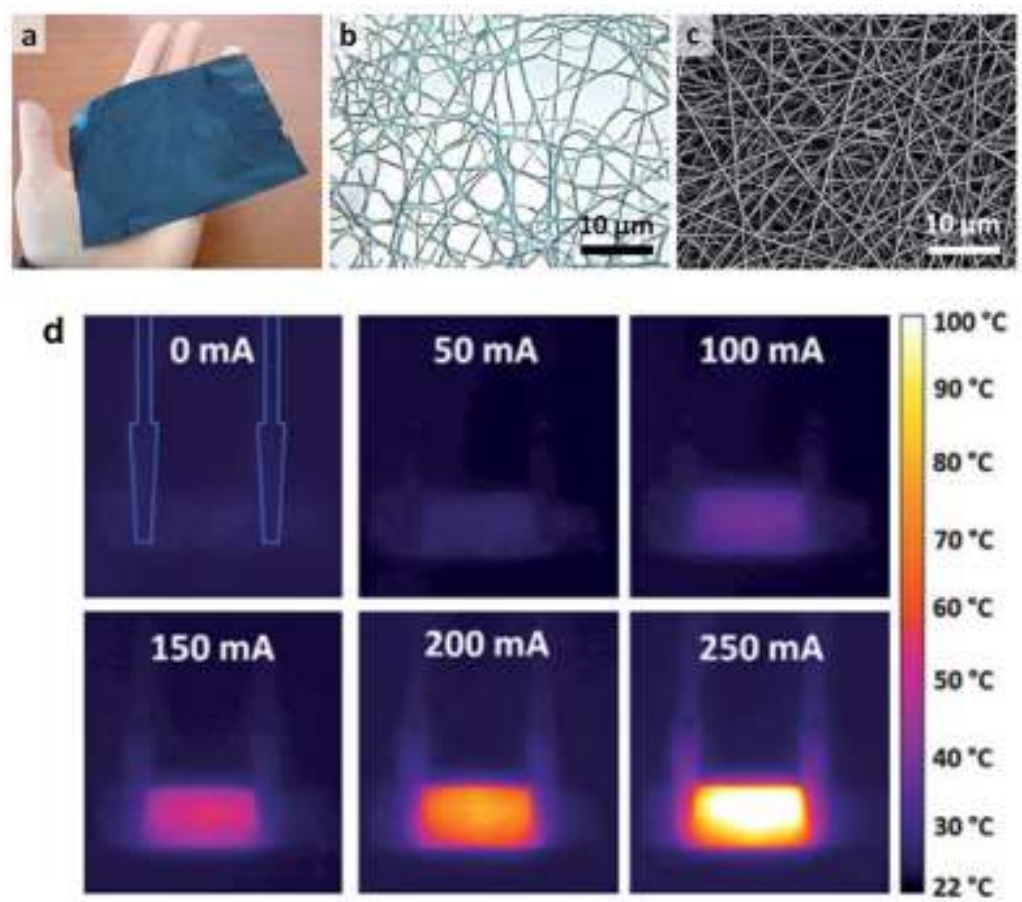

Fig. 7 Electrically colour-changing nanofibers with resistive heating properties. PEDOT nanofiber mat (a), and corresponding optical microscopy (b) and scanning electron microscopy (SEM, c) images. These nanofibers have been realized by electrospinning of a solution with an oxidant (iron(III)tosylate), polyvinylpyrrolidone, and pyridine, followed by vapour-phase polymerization of the EDOT monomer. (d) Mats imaged, at different applied currents, by means of an infrared camera highlighting temperature changes. The scheme of electrodes is shown in the first panel. Adapted with permission from J Mater Chem 2010;20:8233-5 [112]. Copyright (C 2010 National Research Council Canada. Published by The Royal Society of Chemistry. 

(2015).
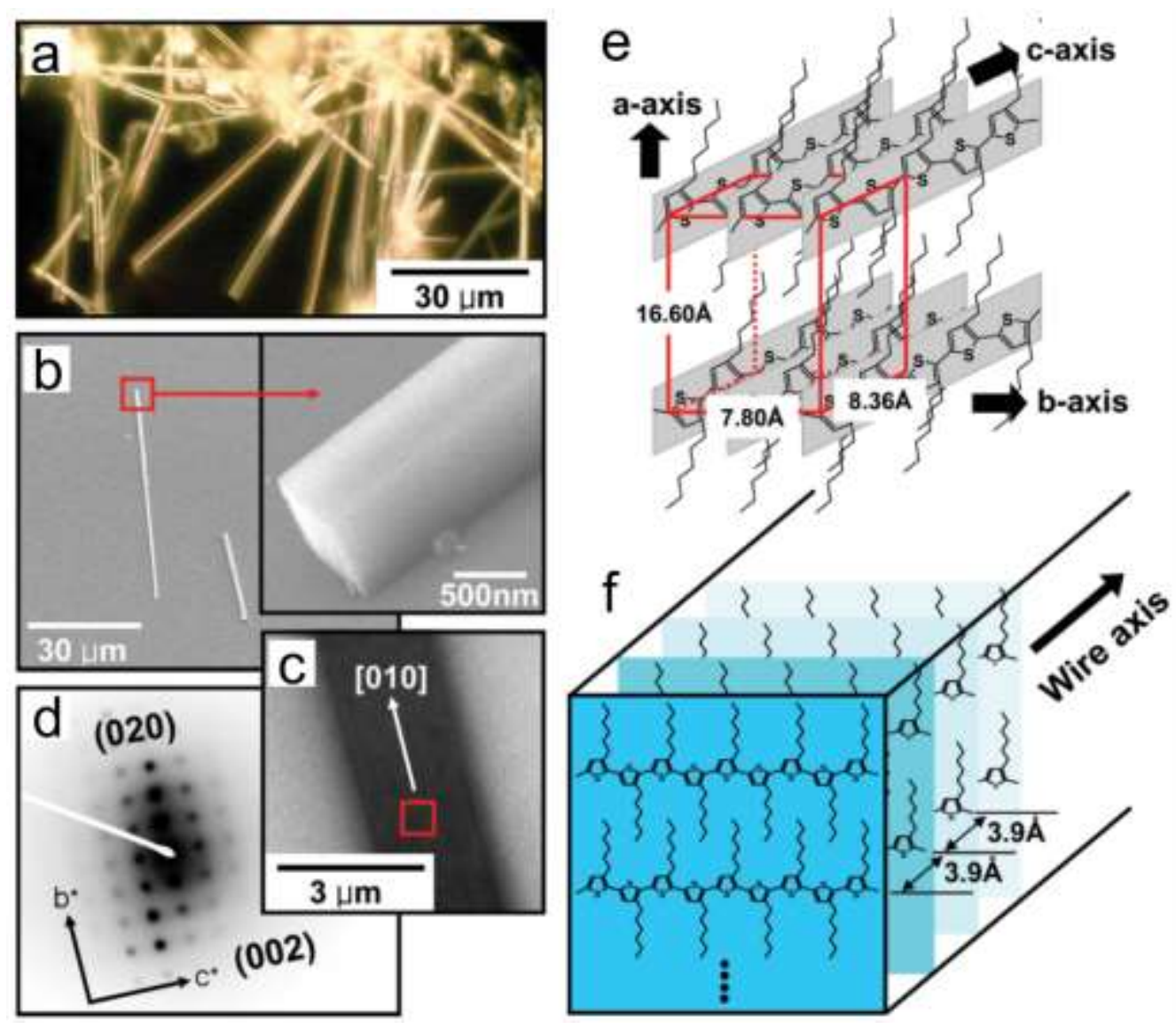

Fig. 8 Morphology and structure of P3HT microfibers. (a) Polarized optical micrograph. (b) SEM and zoom magnification of the tip. (c) Transmission electron microscopy (TEM), highlighting a preferential growth along the [010] direction. (d) Selected-area electron diffraction pattern. (e) Supramolecular structure. (f) Configuration in the fibers. The $b$ axis is aligned along the fiber length. Adapted with permission from Adv Mater 2006;18:719-23 [40]. Copyright $\odot 2006$ Wiley-VCH Verlag GmbH \& Co. KGaA. 
Published in Progress in Polymer Science 43, 48-95, doi: 10.1016/j.progpolymsci.2014.10.001 (2015).
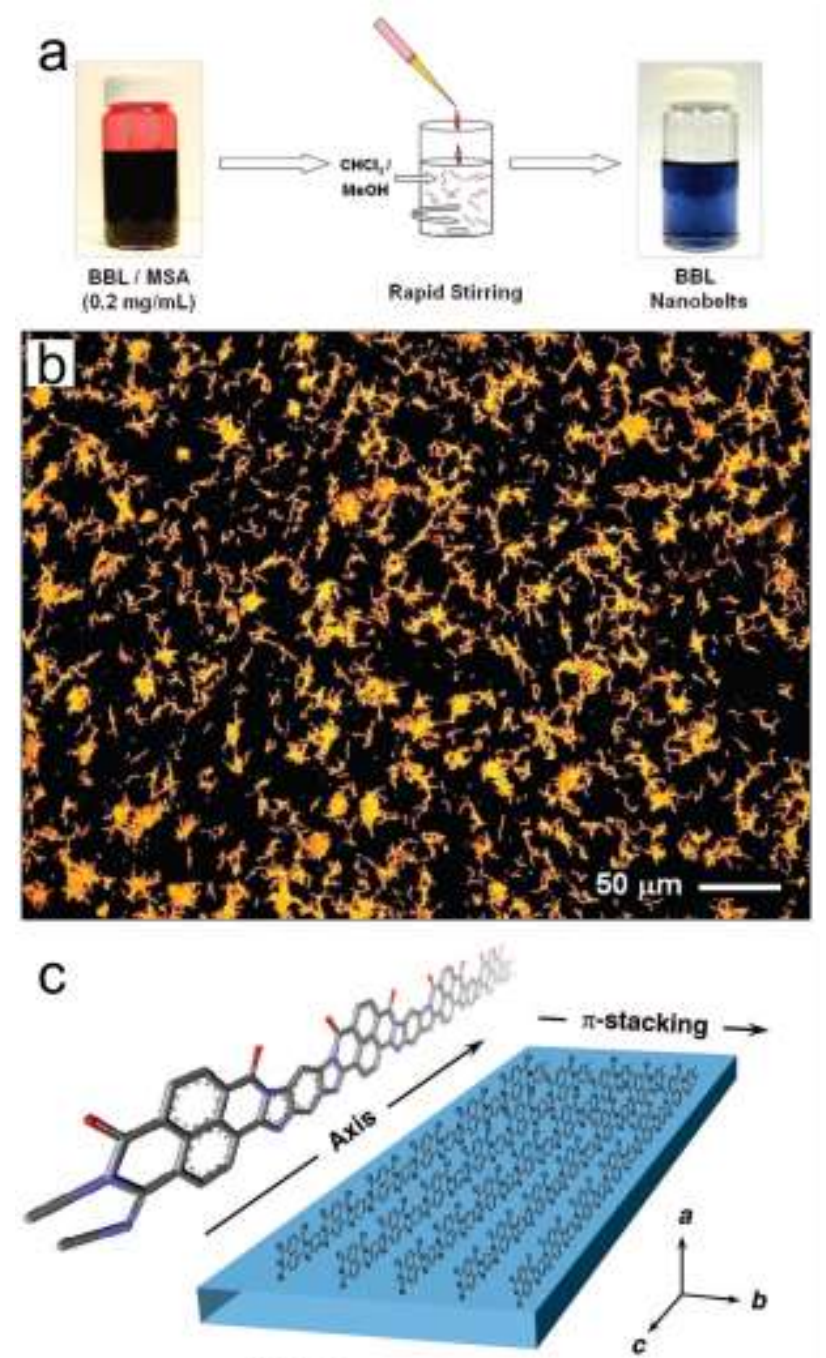

BBL Nanobelt

Fig. 9 Process for preparing BBL nanobelts from methanesulfonic acid (MSA) solutions added dropwise to a rapidstirring vial of $\mathrm{CHCl}_{3} / \mathrm{MeOH}$ (4:1), washing and centrifugation with deionized water (a), dark-field optical micrograph of the resulting nanobelts (b), and schematics of the proposed packing of polymer molecules within an individual nanostructure, with the $\pi$-stacking direction perpendicular to the nanobelt longitudinal axis (c). Adapted with permission from Chem Mater 2008;20:4712-9 [144]. Copyright @ 2008 American Chemical Society. 
Published in Progress in Polymer Science 43, 48-95, doi: 10.1016/j.progpolymsci.2014.10.001 (2015).
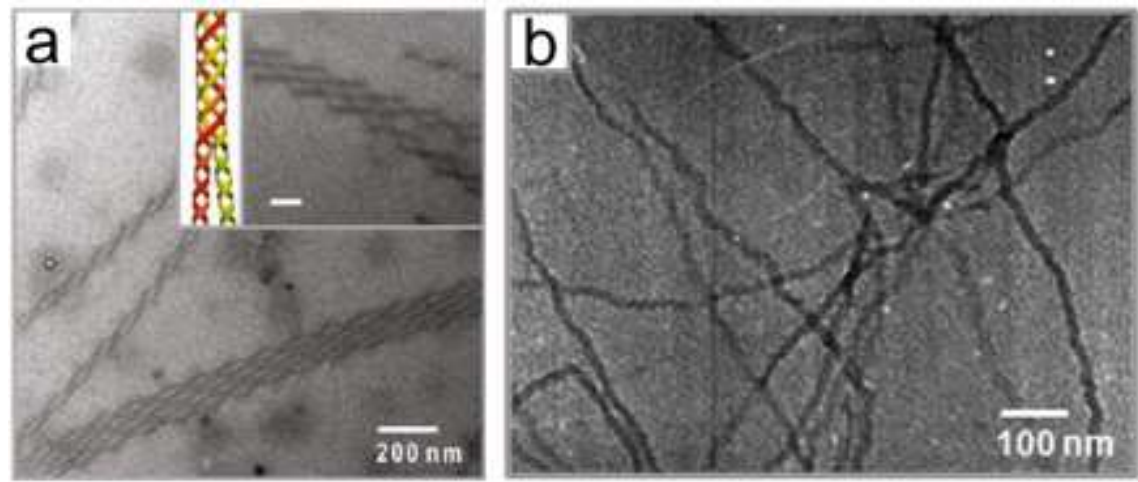

Fig. 10 TEM micrographs showing the formation of helices in multiple strands (a) and the formation of twisted fibrils (b) from P3HT-block-poly(3-triethylene glycol thiophene) copolymers. The inset in (a) displays a TEM micrograph at high resolution (scale bar $=100 \mathrm{~nm}$ ), and a scheme illustrating the formation of a multiple strand. Adapted with permission from J Am Chem Soc 2011;133:10390-3 [146]. Copyright @ 2011 American Chemical Society.
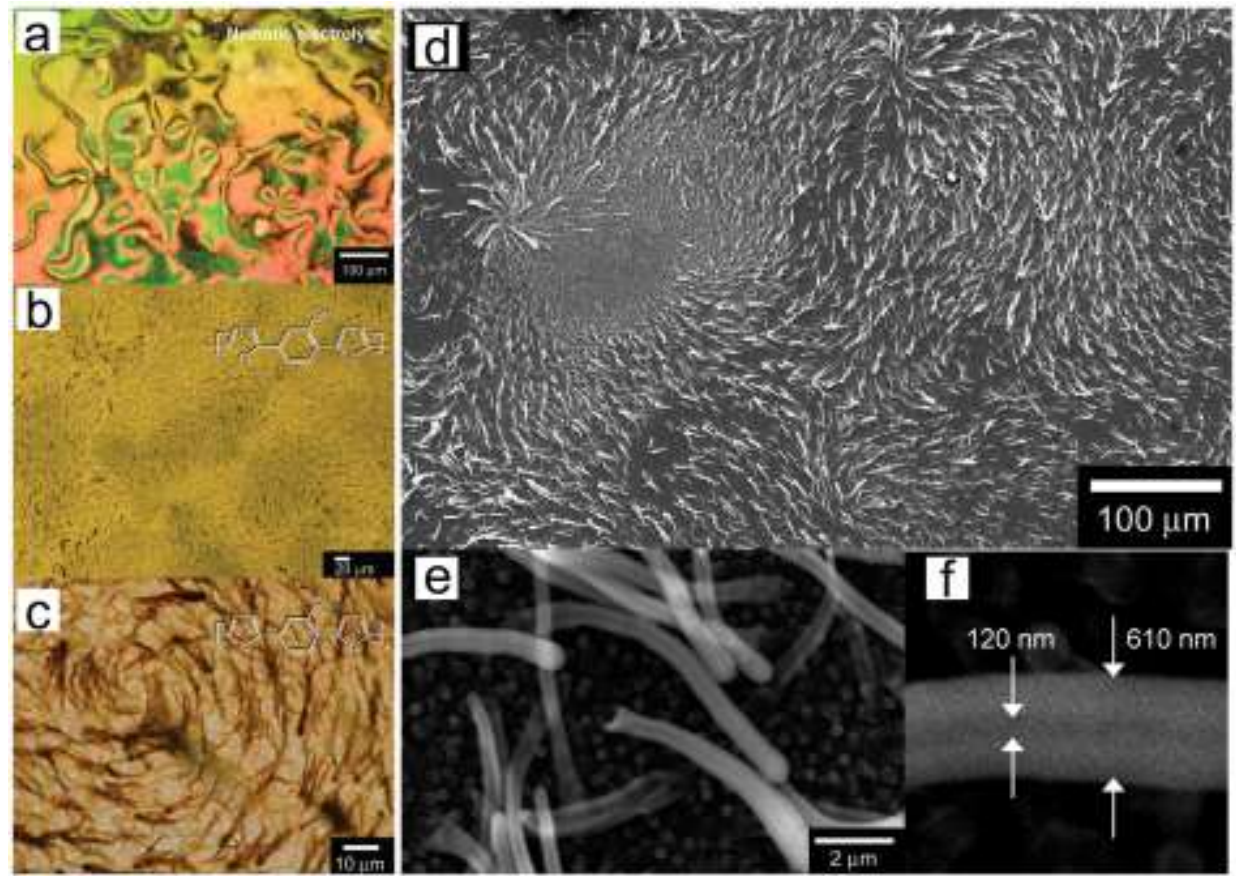

Fig. 11 Achievement and alignment of conjugated polymer nanotubes by a nematic liquid crystal template. Polarized optical micrographs of a 4-cyano-4'-hexylbiphenyl-based nematic electrolyte (a), and of poly[2,5-di(2-furyl)toluene] obtained by electrochemical polymerization in the electrolyte $(b, c)$. (d-f) SEM micrographs of produced nanotubes at different magnifications. Adapted with permission from Macromolecules 2008;41:4551-4 [151]. Copyright @ 2008 American Chemical Society. 
Published in Progress in Polymer Science 43, 48-95, doi: 10.1016/j.progpolymsci.2014.10.001 (2015).
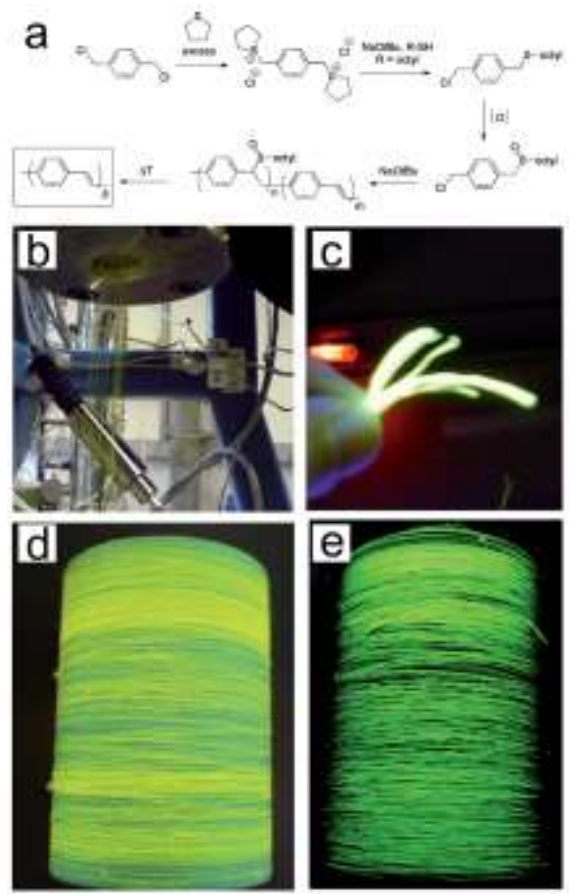

Fig. 12 Sulfinyl-based PPV fibers realized by dry-spinning. (a) Polymer synthesis. (b) Photograph of the dry-spinning set-up. (c) Produced fibers under UV irradiation. (d, e) Reel-collected fibers under ambient light (d) and under UV (e), respectively. Adapted with permission from J Mater Chem 2012;22:11851-60 [29]. Copyright @ 2012 The Royal Society of Chemistry.

a
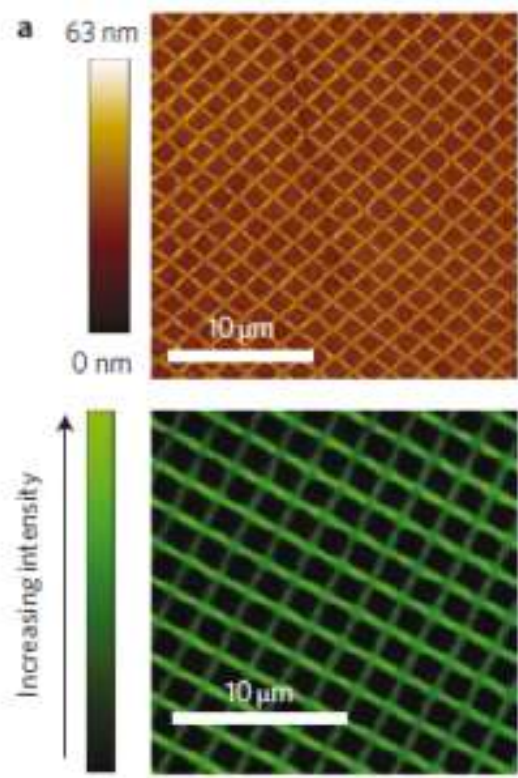

b
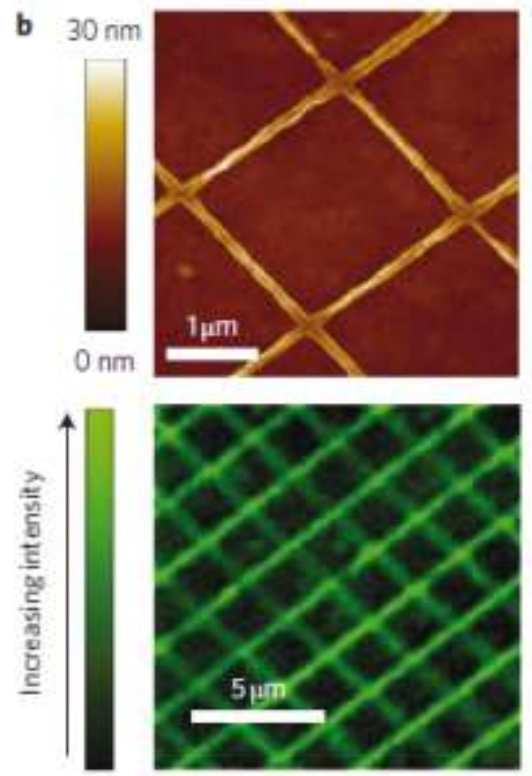

Fig. 13 AFM micrographs (top panels) and confocal fluorescence images (bottom panels) of PPV nanowires fabricated by thermochemical nanopatterning. The nanostructures are obtained by polymerizing at $230{ }^{\circ} \mathrm{C}$ with a probe tip moving at a speed of $10 \mu \mathrm{m} \mathrm{s}^{-1}$ (a), and at $250{ }^{\circ} \mathrm{C}$ with a probe tip speed of $5 \mu \mathrm{m} \mathrm{s}^{-1}$ (b), respectively. Adapted with permission from Nat Nanotechnol 2009;4:664-8 [30]. Copyright @ 2009 McMillan Publishers Ltd. 
Published in Progress in Polymer Science 43, 48-95, doi: 10.1016/j.progpolymsci.2014.10.001 (2015).
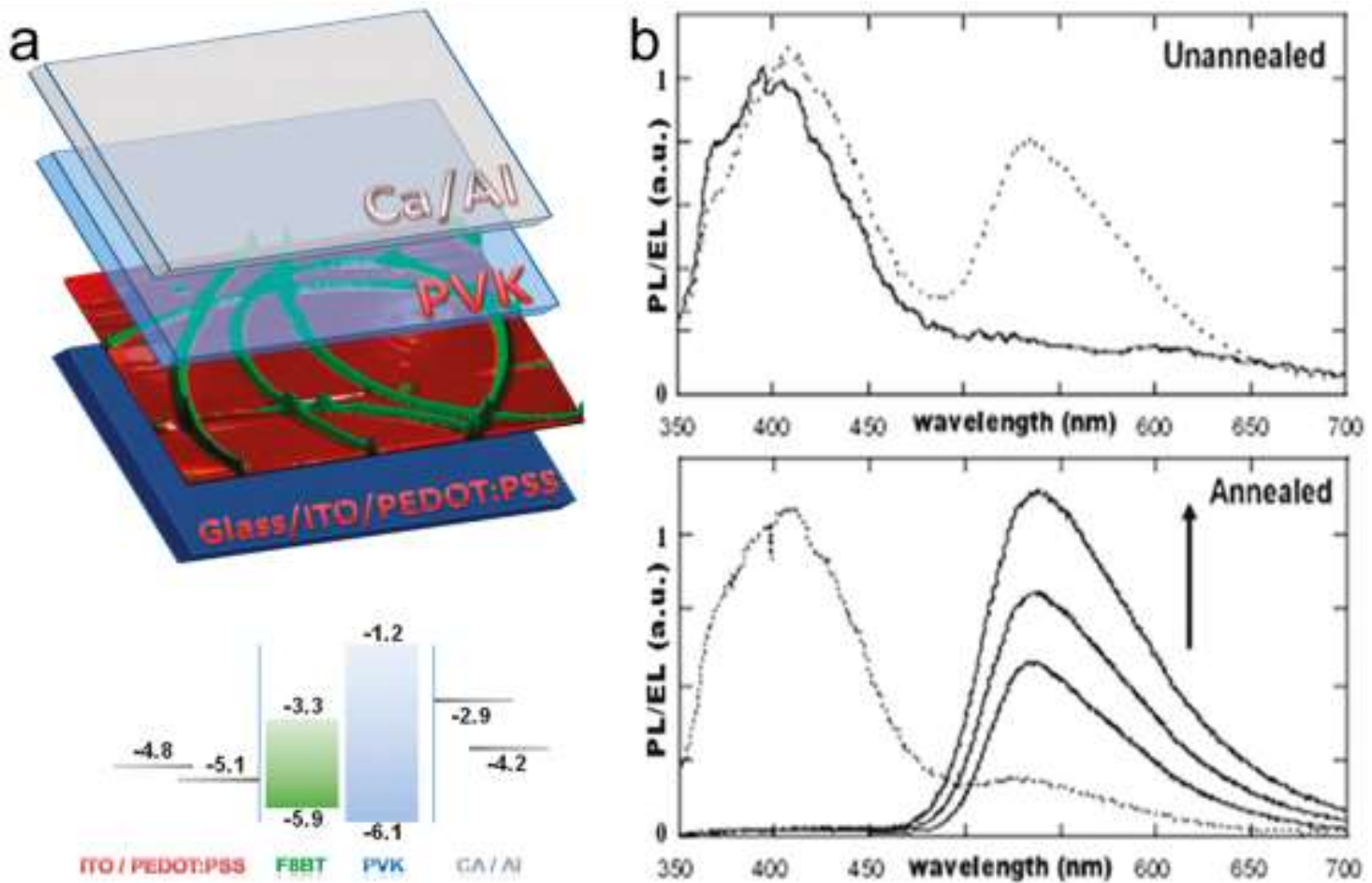

Fig. 14 Building OLEDs by electrospun polymer nanofibers. (a) Device structure and energy level scheme. (b) Normalized electroluminescence (solid lines) and photoluminescence (dotted lines) spectra of F8BT/PEO fibers in an unannealed (top panel) and in an annealed (bottom panel) condition, respectively. Annealing has been carried out at $150^{\circ} \mathrm{C}$, leading to separation of the F8BT and PEO components and significantly improving electroluminescence. Adapted with permission from ACS Nano 2011;5:5572-8 [61]. Copyright @ 2011 American Chemical Society. 
Published in Progress in Polymer Science 43, 48-95, doi: 10.1016/j.progpolymsci.2014.10.001 (2015).

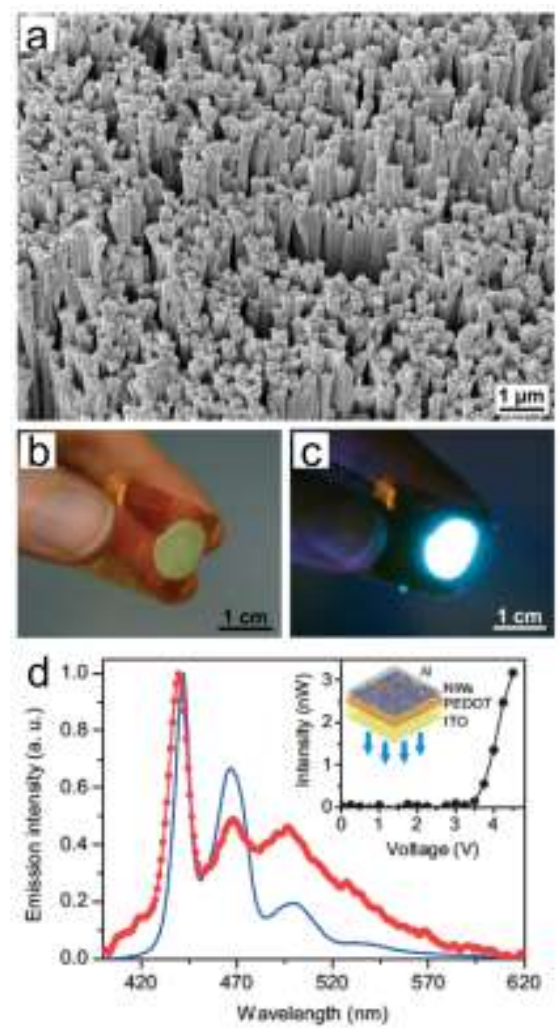

Fig. 15 OLEDs by conjugated polymer nanowires synthetized in hard templates. (a) SEM micrograph of vertically standing PFO nanowires after template removal. (b, c) Photographs of the array, mounted on a flexible tape and illuminated by white light (b) or under UV excitation (c), respectively. (d) Electroluminescence (dotted line) and photoluminescence (continuous line) spectra. Inset: device scheme and emission intensity-voltage characteristics. Adapted with permission from Adv Mater 2008;20:42-8 [56]. Copyright @ 2008 Wiley-VCH Verlag GmbH \& Co. KGaA.
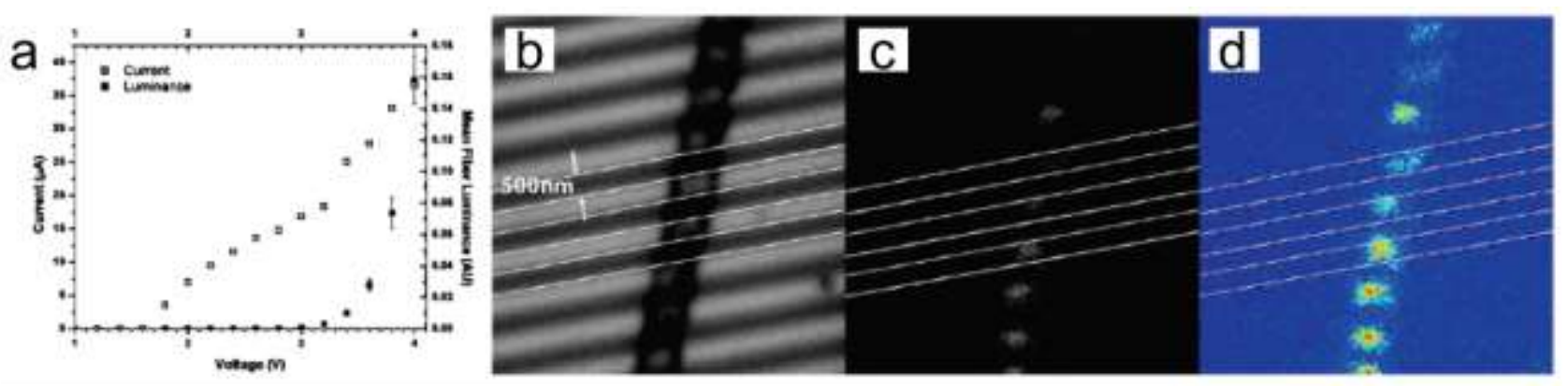

Fig. 16 OLEDs by electrospun polymer nanofibers and a planar electrode geometry. (a) Nanofiber device emission luminance-voltage (solid symbols) and current-voltage (open symbols) characteristics. (b) Bright field micrograph of an electrospun fiber made of ruthenium(II) tris(bipyridine)-doped PEO and spanning several interelectrode gaps, each with width of $500 \mathrm{~nm}$. (c) Corresponding emission map at $4 \mathrm{~V}$, also shown in pseudo-colors in (d). Adapted with permission from Nano Lett 2007;7:458-63 [69]. Copyright @ 2007 American Chemical Society. 
Published in Progress in Polymer Science 43, 48-95, doi: 10.1016/j.progpolymsci.2014.10.001 (2015).

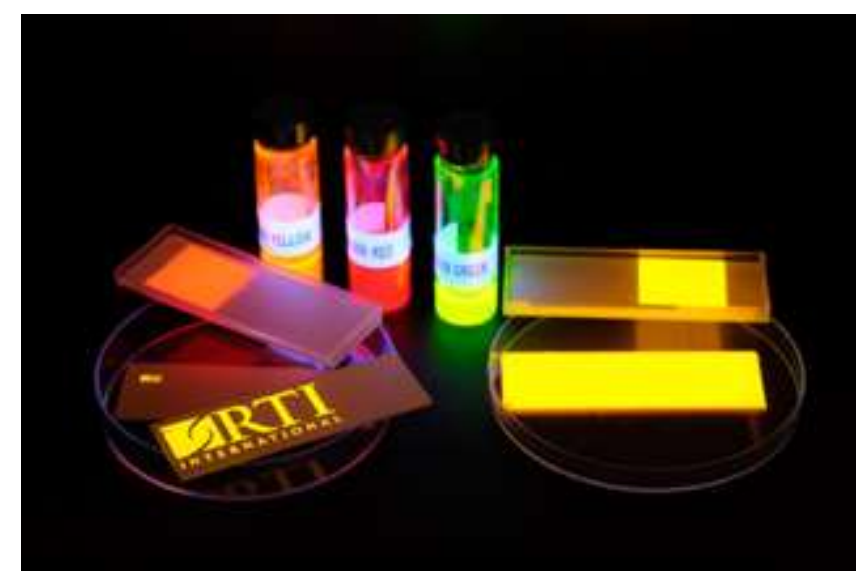

Fig. 17 Pristine solutions and surfaces covered by NLITe ${ }^{\mathrm{TM}}$ light-emitting nanofibers. Photograph kindly supplied by RTI International.

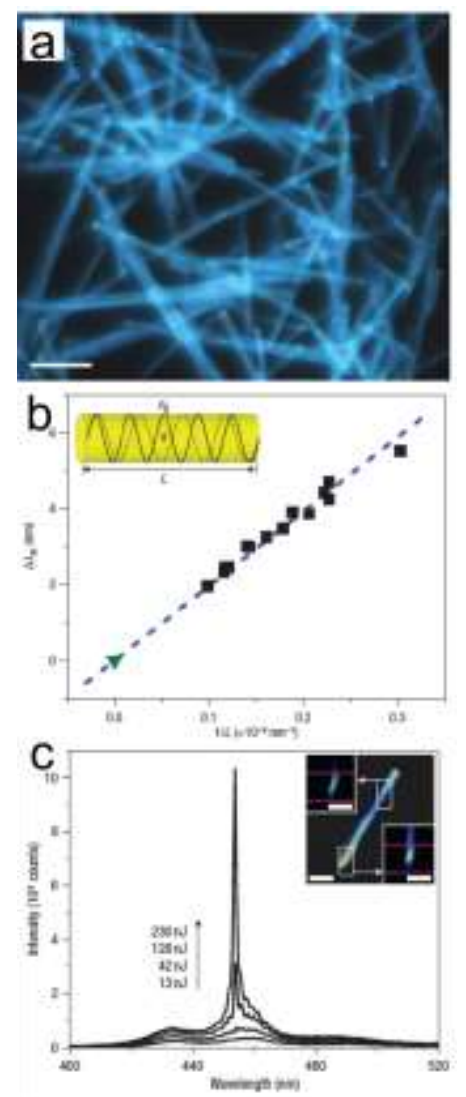

Fig. 18 Conjugated polymer lasing nanowires grown by hard templates. (a) Fluorescence micrograph. (b) Dependence of the spacing between emission modes on the reciprocal wire length, $1 / L$, evidencing good agreement with Fabry-Pérot microcavity mechanisms at the base of spectral selection (dashed line). The inset is a schematics of the Fabry-Pérot microcavity defined by the tips of the organic wire. (c) Room temperature lasing spectra. The inset shows an emission micrograph of the wire, highlighting the different regions where spectra have been collected. Scale bar $=2 \mu \mathrm{m}$. Adapted with permission from Nat Nanotechnol 2007;2:180-4 [54]. Copyright @ 2007 McMillan Publishers Ltd. 
Published in Progress in Polymer Science 43, 48-95, doi: 10.1016/j.progpolymsci.2014.10.001 (2015).
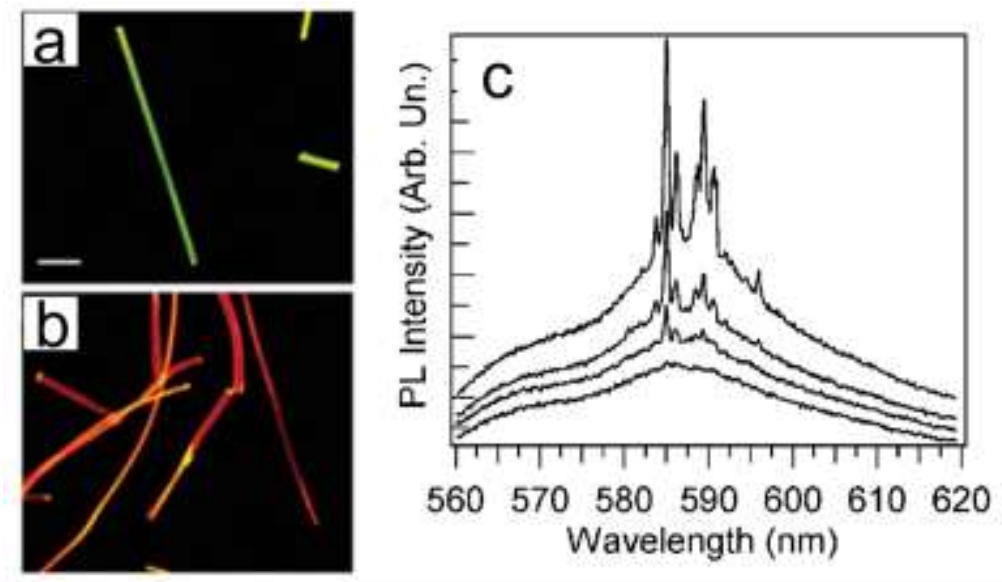

Fig. 19 Dye-doped, electrospun lasing polymer nanofibers. (a, b) Fluorescence micrographs of polymer nanofibers doped by coumarin and rhodamine, respectively. (c) Room temperature lasing spectra collected at different excitation

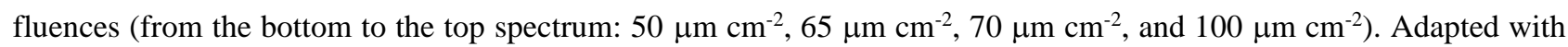
permission from Small 2009;5:562-6 [68]. Copyright @ 2009 Wiley-VCH Verlag GmbH \& Co. KGaA.

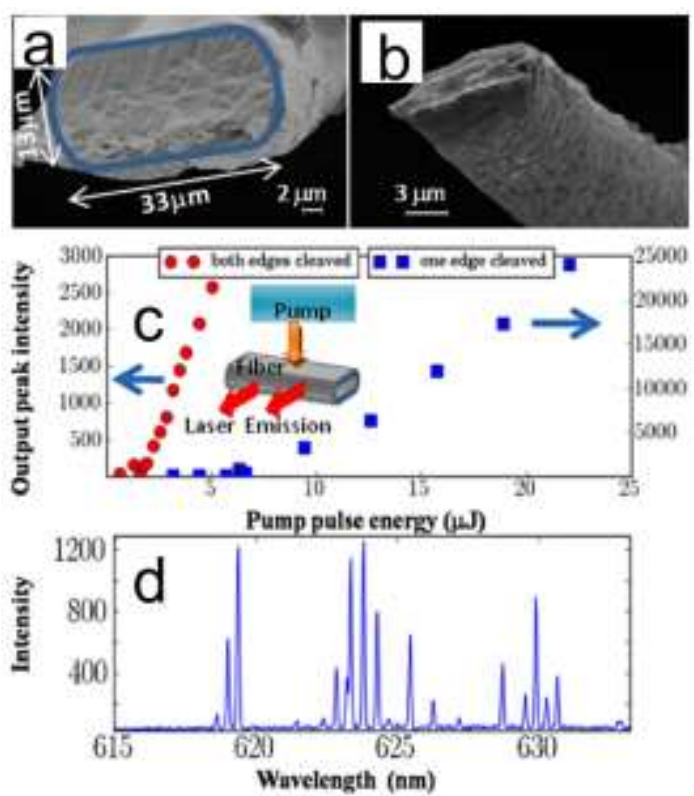

Fig. 20 Electrospun polymer fiber doped with DCM. (a, b) SEM micrographs highlighting the non-circular crosssections of the fibers. (c) Input-output characteristics of the optically-pumped lasing fibers. The inset displays the scheme of the pumping and collection geometries. (d) Typical lasing spectrum. Adapted with permission from Appl Phys Lett 2011;99:263303/1-3 [257]. Copyright (C 2011 AIP Publishing LLC. 
Published in Progress in Polymer Science 43, 48-95, doi: 10.1016/j.progpolymsci.2014.10.001 (2015).

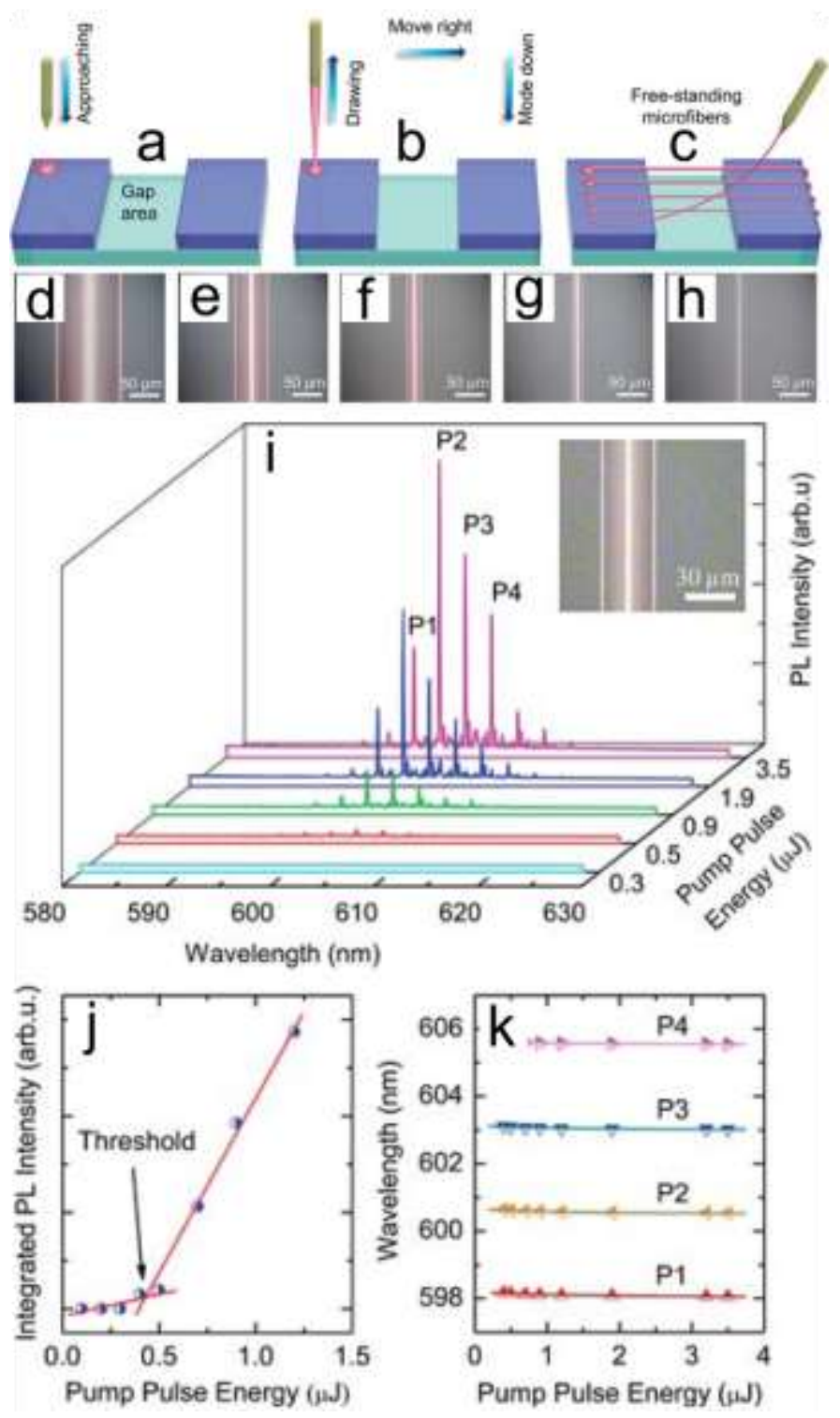

Fig. 21 Polymer fiber production by drawing through a gap of $0.5 \mathrm{~cm}(\mathrm{a}-\mathrm{c})$, top-view optical micrographs of obtained fibers having different diameters (d-h), room temperature lasing spectra from whispering gallery modes (i), input-output characteristics (j), and dependence of the emission peaks (P1-P4 in i) on the pump pulse energy (k). The inset in (i) shows another top-view optical micrograph of the lasing fiber. Adapted with permission from Laser Photon Rev 2013;7:133-9 [258]. Copyright @ 2012 Wiley-VCH Verlag GmbH \& Co. KGaA. 
Published in Progress in Polymer Science 43, 48-95, doi: 10.1016/j.progpolymsci.2014.10.001 (2015).

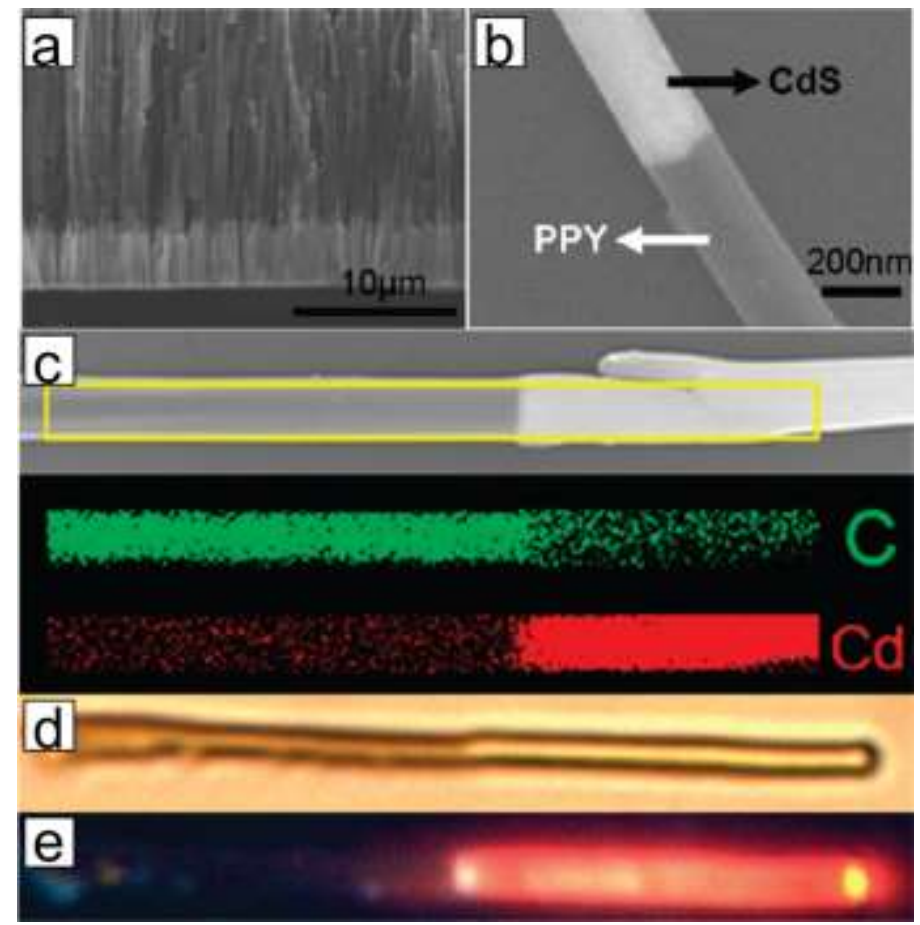

Fig. 22 Organic-inorganic $p-n$ junction nanowires. Side-view (a) and magnified SEM view (b) of CdS-PPY heterojunction nanowires. (c) Element mapping and (d) optical micrograph of a single wire. (e) Corresponding fluorescence micrograph. Adapted with permission from J Am Chem Soc 2008;130:9198-9 [190]. Copyright @ 2008 American Chemical Society. 
Published in Progress in Polymer Science 43, 48-95, doi: 10.1016/j.progpolymsci.2014.10.001 (2015).
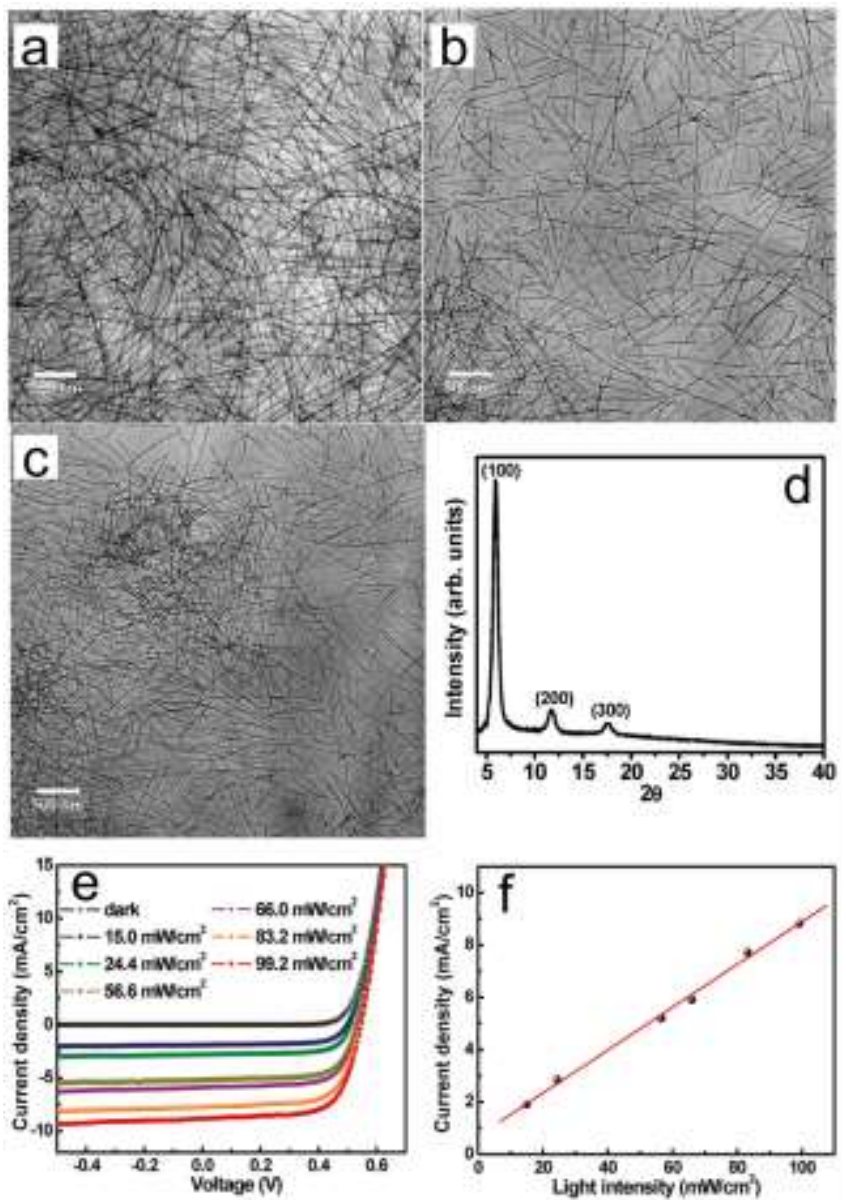

Fig. 23 (a-c) TEM micrographs of regioregular poly(3-pentylthiophene) nanowires, self-assembled at different concentrations in 1,2-dichlorobenzene, (d) exemplary X-ray diffraction spectrum, and (e, f) photovoltaic performances. (e) Current density-voltage characteristics of a nanowire:fullerene bulk heterojunction solar cell in the dark and under AM1.5 solar illumination in air at different light intensity. (f) Current density vs. light intensity. Adapted with permission from Macromolecules 2009;42:8817-26 [269]. Copyright @ 2009 American Chemical Society. 
Published in Progress in Polymer Science 43, 48-95, doi: 10.1016/j.progpolymsci.2014.10.001 (2015).
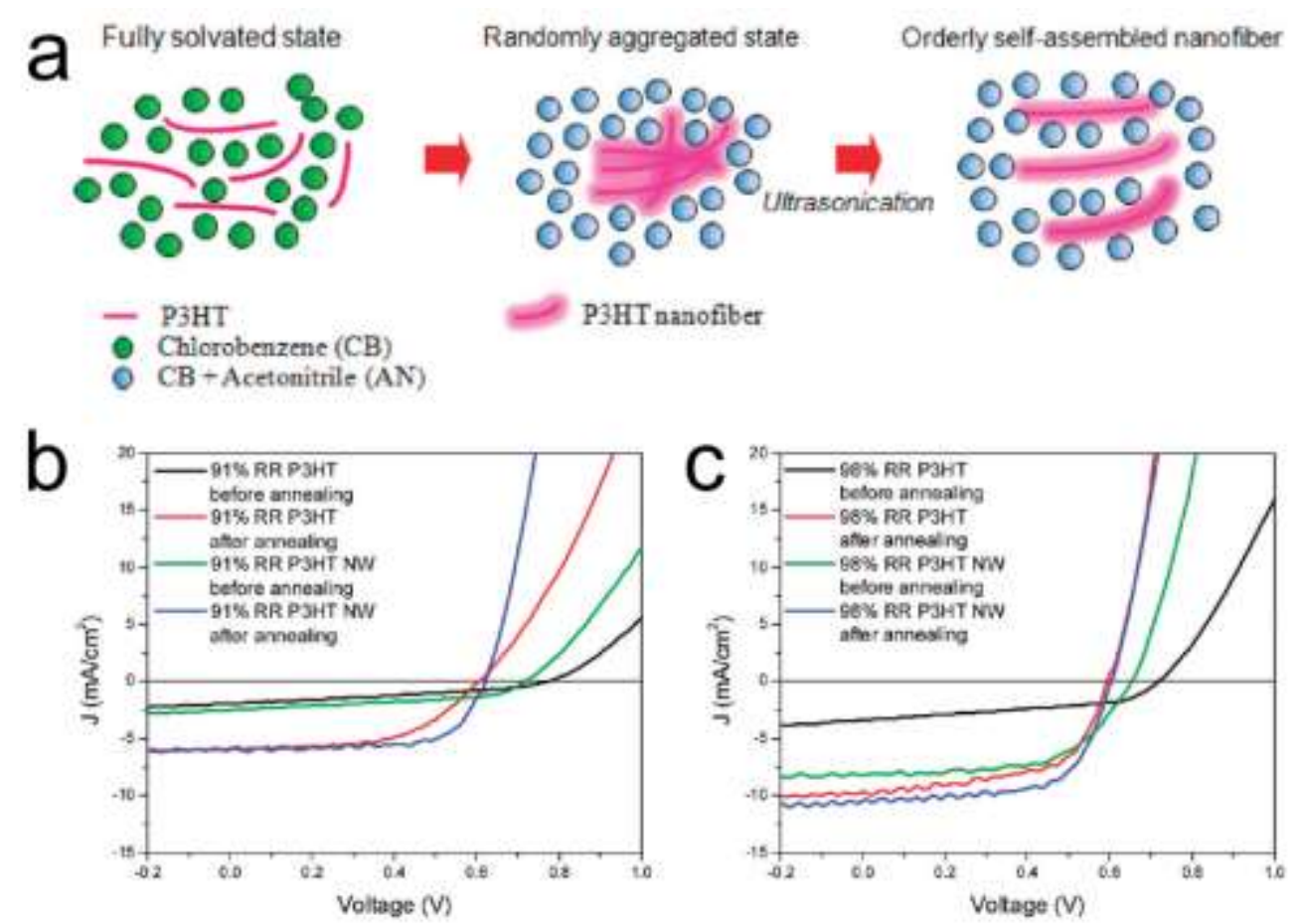

Fig. 24 Formation of P3HT nanofibers assisted by ultrasonication, and use of so obtained fibers in solar cells. (a) Scheme showing the assembly of nanofibers in a system comprising a dipolar solvent. (b, c) Current density-voltage characteristics of P3HT:fullerene and P3HT nanofiber (here, NW):fullerene bulk heterojunction solar cells, before and after thermal annealing and under $100 \mathrm{~mW} \mathrm{~cm}^{-2}$, AM1.5 solar illumination. The regioregularity of P3HT is $91 \%$ (b) and 98\% (c), respectively. Adapted with permission from ACS Nano 2010;4:2160-6 [272]. Copyright (C) 2010 American Chemical Society. 
Published in Progress in Polymer Science 43, 48-95, doi: 10.1016/j.progpolymsci.2014.10.001 (2015).
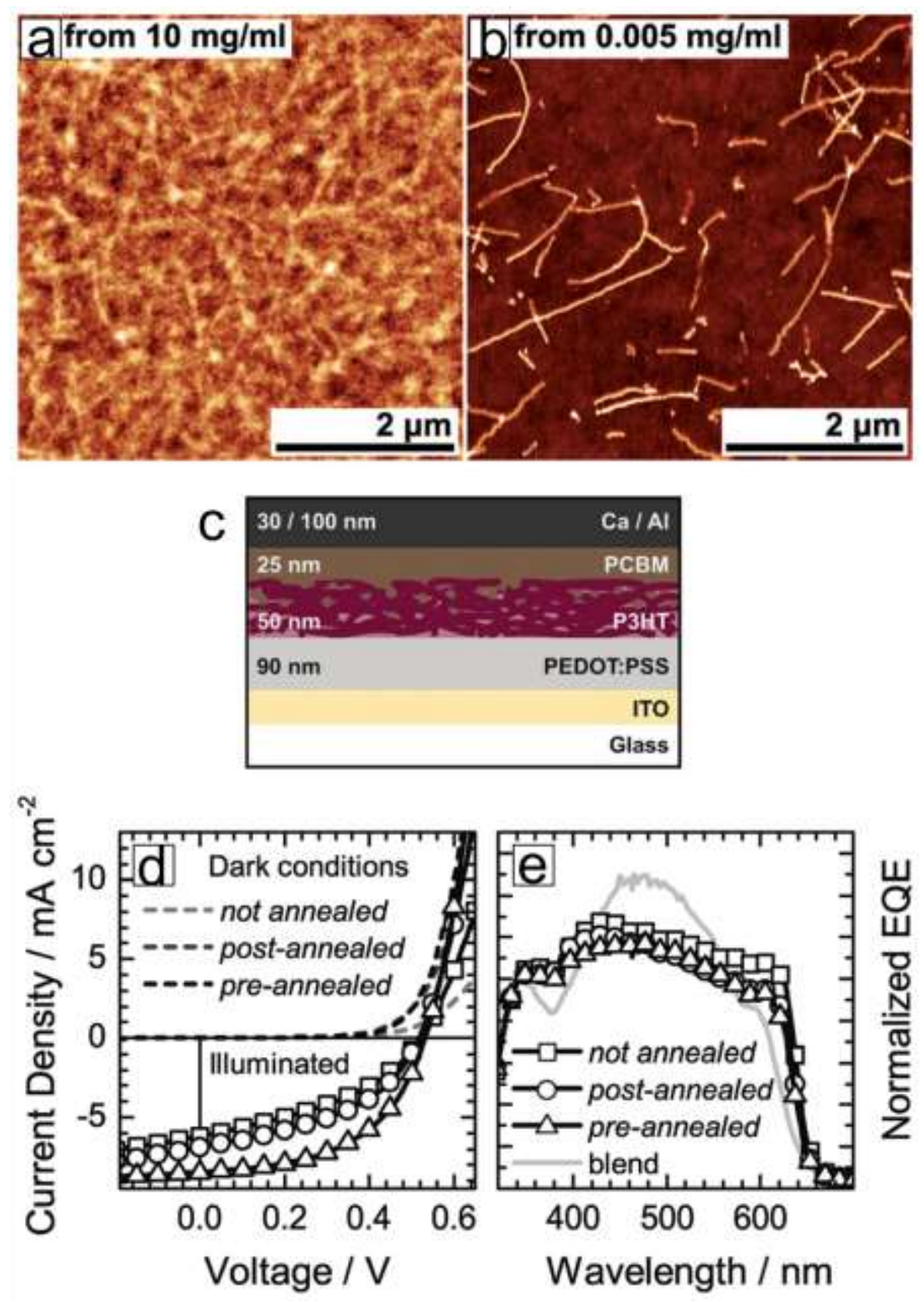

Fig. 25 Organic solar cells based on P3HT nanofibers formed by addition of di-tert-butyl peroxide to a solution in chlorobenzene. (a, b) AFM micrographs of P3HT films comprising nanofibers, following different dilutions of the cast solutions. (c) Device scheme. (d) Current density-voltage characteristics of the solar cells, before and after thermal annealing, under dark and under $100 \mathrm{~mW} \mathrm{~cm}{ }^{-2}$, AM1.5 solar illumination. (e) Measured external quantum efficiency (here EQE) spectra. Adapted with permission from J Mater Chem C 2013;1:7748-57 [274]. Published by The Royal Society of Chemistry. 
Published in Progress in Polymer Science 43, 48-95, doi: 10.1016/j.progpolymsci.2014.10.001 (2015).

(a)
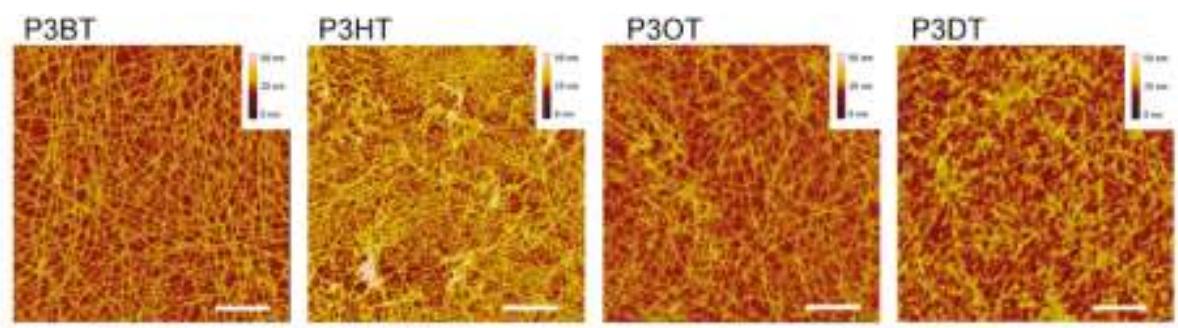

(b)
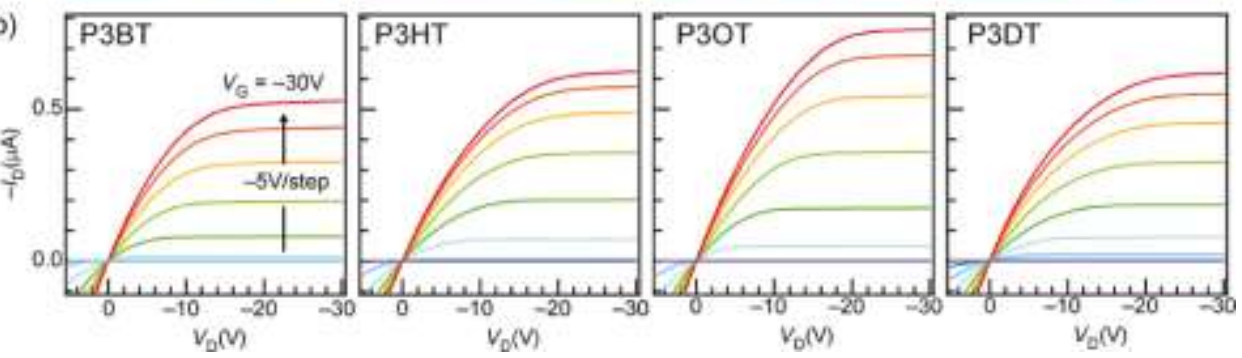

Fig. 26 AFM micrographs of deposited nanofibers made of P3ATs with varying alkyl chain lengths (a), and corresponding FET characteristics (b). Poly-3-butylthiophene, poly-3-octylthiophene, and poly-3-decylthiophene are abbreviated as P3BT, P3OT and P3DT, respectively. Reproduced with permission from Macromolecules 2010;43:7891-4 [33]. Copyright @ 2010 American Chemical Society.
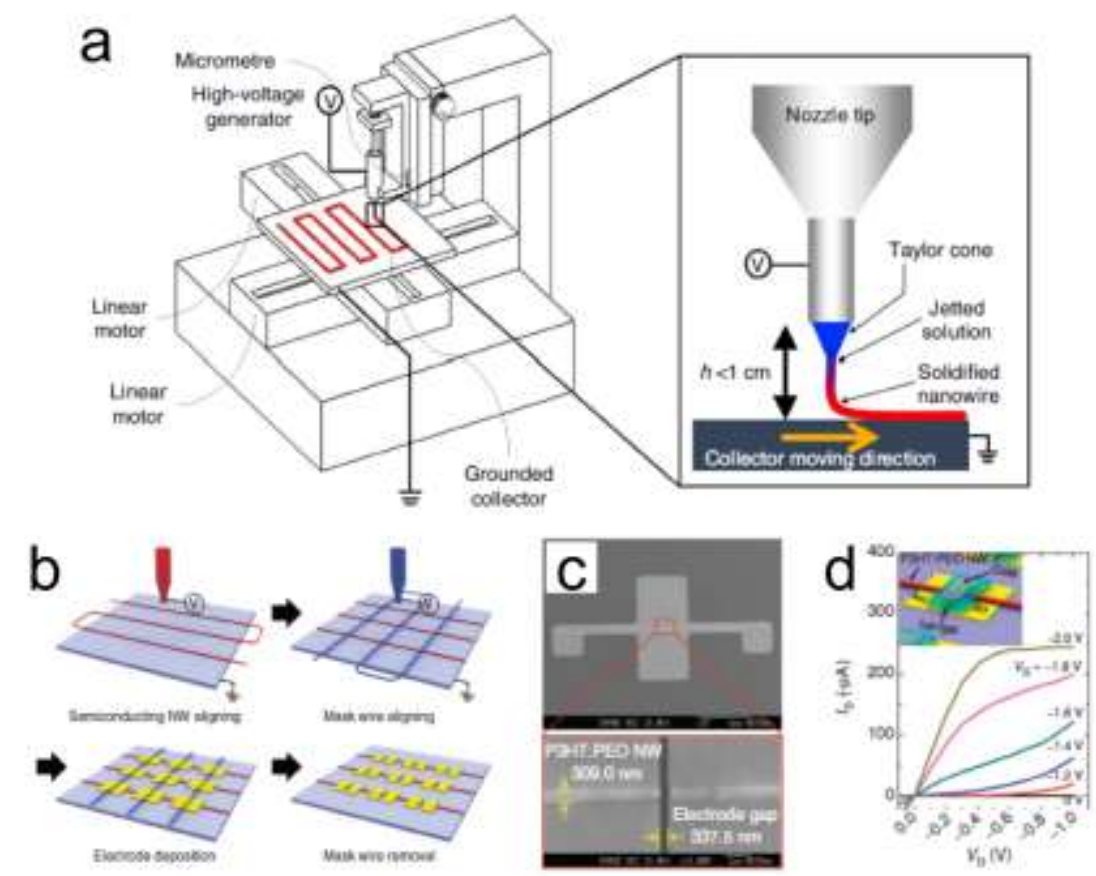

Fig. 27 Conductive polymer nanowire printing and electronic device production. (a) Scheme of the developed printing set-up and process. (b) FET fabrication steps. (c) SEM micrographs of a P3HT/PEO nanowire and of the device electrode gap. (d) FET characteristics. Inset: three-dimensional device scheme. Adapted with permission from Nat Commun 2013;4:1773/1-9 [49]. Copyright @ 2013 McMillan Publishers Ltd. 
Published in Progress in Polymer Science 43, 48-95, doi: 10.1016/j.progpolymsci.2014.10.001 (2015).
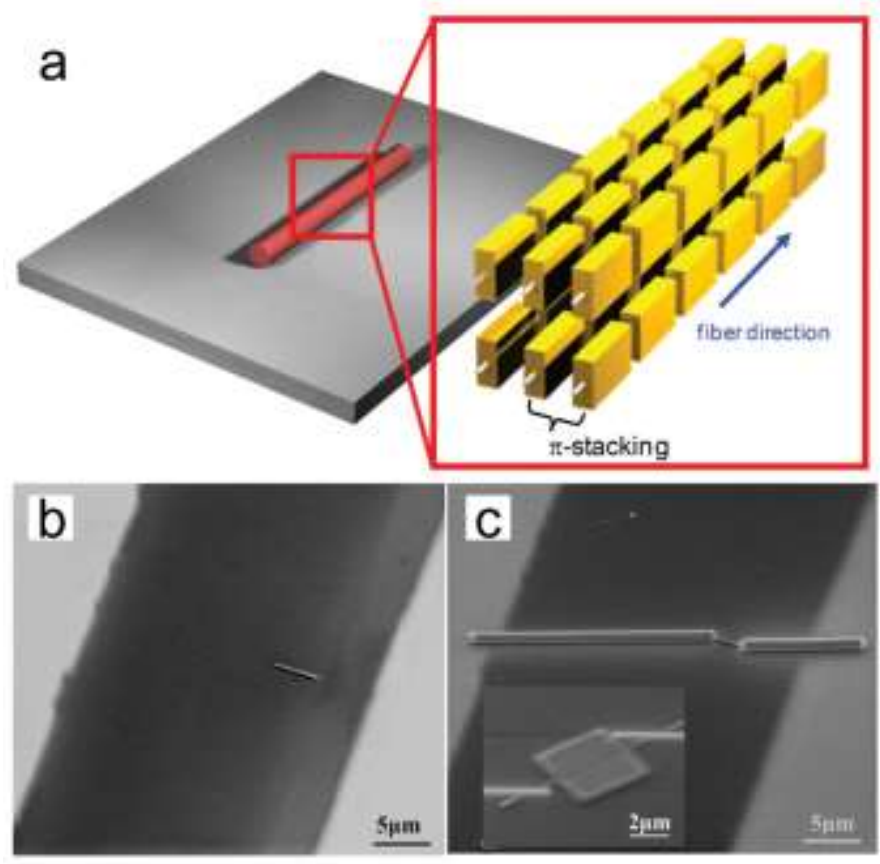

Fig. 28 Schematics of the macromolecular and $\pi$-stacking direction of cyclopentadithiophene-benzothiadiazole in nanofibers (a), and SEM micrographs of the fiber between electrodes before (b) and after (c) deposition of Pt highresolution features by a focused ion beam. Adapted with permission from Adv Mater 2012;24:417-20 [138]. Copyright (c) 2012 Wiley-VCH Verlag GmbH \& Co. KGaA.
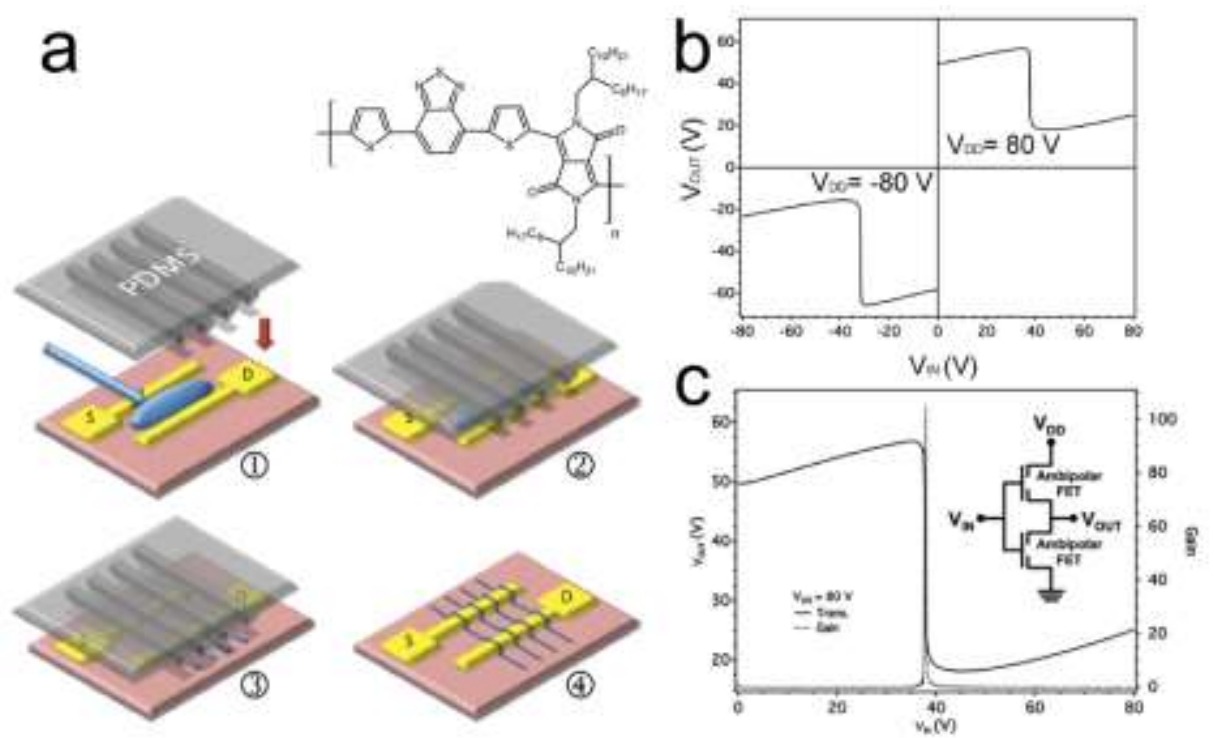

Fig. 29 Schematics of the fabrication of a diketopyrrolopyrrole-benzothiadiazole-based copolymer nanofiber device by lithographically controlled wetting (a), and electrical characteristics (b), transfer characteristic and gain (c) of the soproduced inverter. The insets show the polymer molecular structure (a) and the inverter circuit scheme (c), respectively. Adapted with permission from Nano Lett 2013;13:3643-7 [283]. Copyright @ 2013 American Chemical Society. 
Published in Progress in Polymer Science 43, 48-95, doi: 10.1016/j.progpolymsci.2014.10.001 (2015).
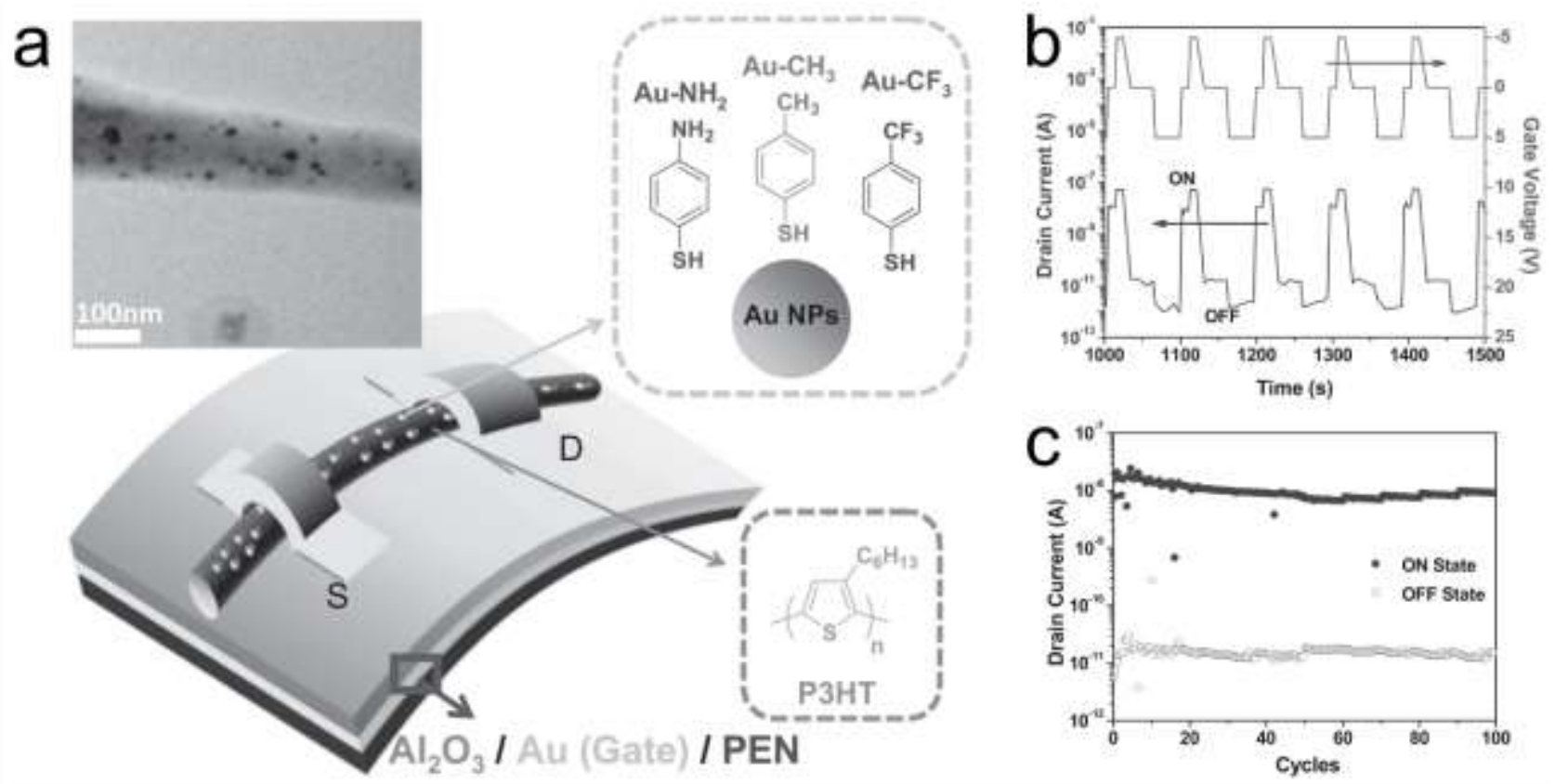

Fig. 30 Scheme of a P3HT:Au- $\mathrm{CF}_{3}$ hybrid nanofiber-based transistor memory device (a), resulting write-read-erase-read cycles (b) and dependence of the drain current on the number of performed cycles, highlighting the device stability (c). Inset in (a): TEM micrograph of a hybrid nanofiber. Adapted with permission from Adv Funct Mater 2013;23:4960-8 [299]. Copyright @ 2013 Wiley-VCH Verlag GmbH \& Co. KGaA.
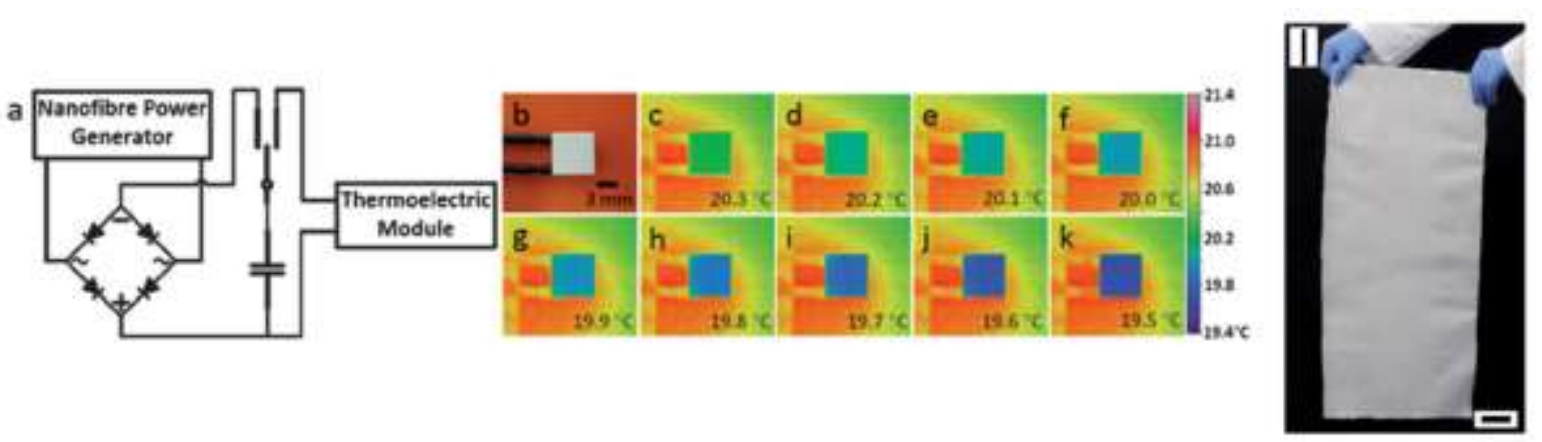

Fig. 31 Nanofiber piezoelectric power generator driving a thermoelectric Peltier cooler, and large-area nanofiber production. Circuit scheme (a), photograph (b) and infrared thermal images (c-k) of the Peltier cooler driven by a nanofiber-based piezoelectric generator, and (l) large-area PVDF mat realized by electrospinning. Scale bar $=5 \mathrm{~cm}$. Adapted with permission from Energy Environ Sci 2013;6:2196-202 [316]. Copyright (c) 2013 The Royal Society of Chemistry. 
Published in Progress in Polymer Science 43, 48-95, doi: 10.1016/j.progpolymsci.2014.10.001 (2015).
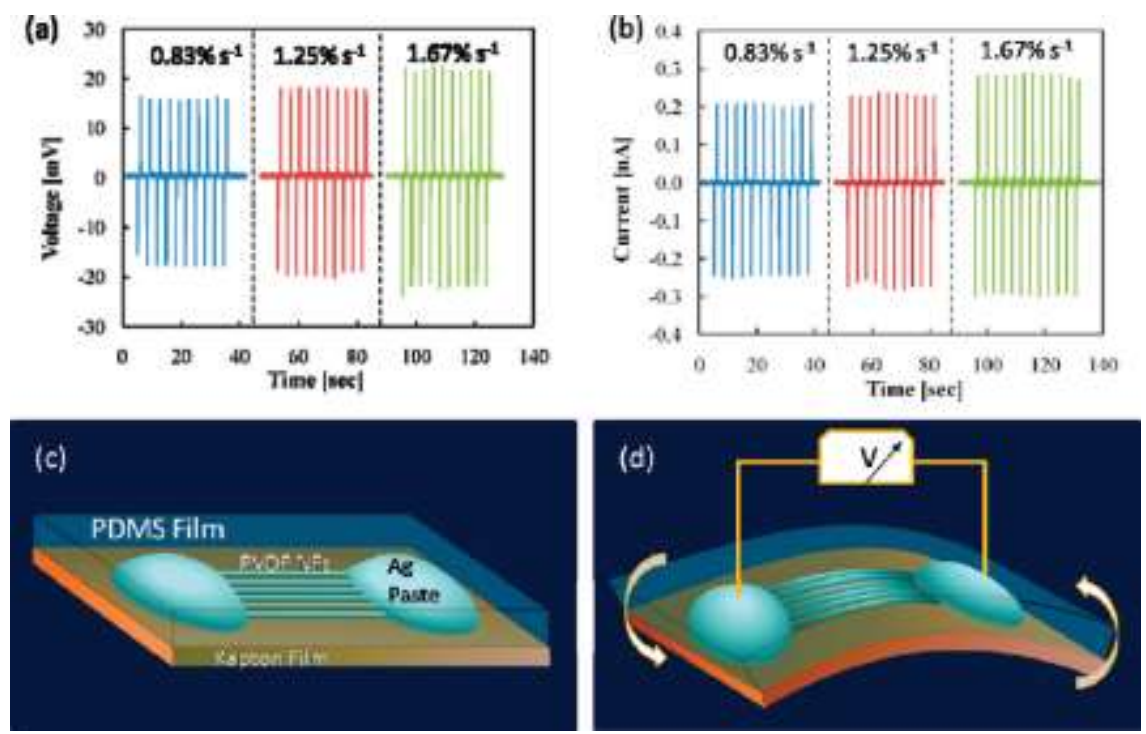

Fig. 32 Open-loop voltage (a) and short-circuit current (b) of a PVDF nanofiber generator, vs. strain cycles, and device scheme in planar conditions (c) and during bending (d). The maximum applied strain is here $0.05 \%$. Voltages up to 20 mV are measured. Reproduced with permission from ACS Nano 2010;4:3647-52 [308]. Copyright @ 2010 American Chemical Society.
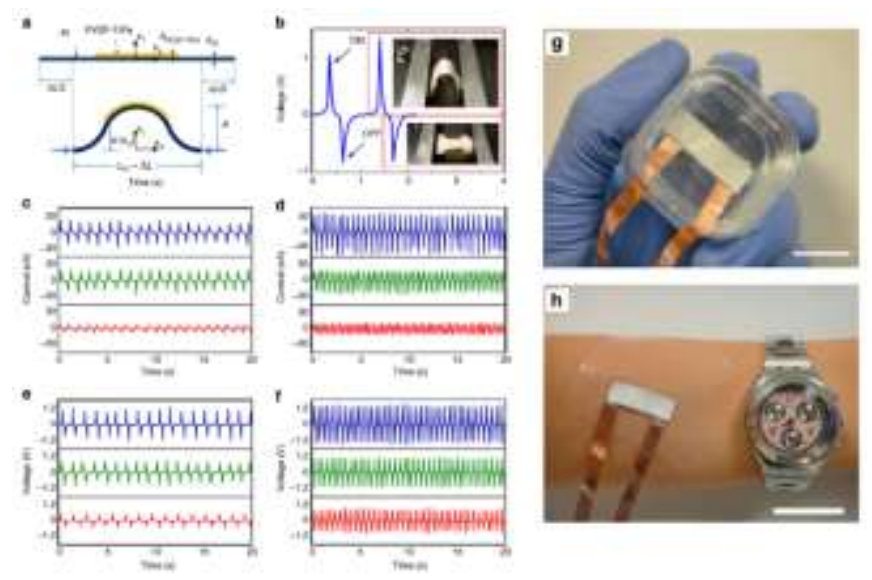

Fig. 33 High-performance piezoelectric arrays of electrospun nanofibers. (a) Device geometry. Here $L_{P I}, \Delta L, w, h_{P V D F}$ $T_{r F e}$ and $h_{P I}$ indicate the initial length of the substrate (flexible polyamide) on which nanofibers are deposited, the inplane substrate compression, the corresponding out-of-plane, buckling displacement (whose maximum value is $A$ ), the thickness of the array of piezoelectric nanofibers, and the thickness of the substrate, respectively. (b) Voltage measured under bending cycles carried out at a frequency of $1 \mathrm{~Hz}$ as shown in the photographs in the insets. (c, d) Short-circuit current and (e, f) open-loop voltage measured during bending cycles at $1 \mathrm{~Hz}(\mathrm{c}, \mathrm{e})$ and at $2 \mathrm{~Hz}(\mathrm{~d}, \mathrm{f})$, respectively. In (cf), panels in each Figure refer to different substrate thickness (from bottom to top panels in each Figure, $h_{P I}=75 \mu \mathrm{m}$, $150 \mu \mathrm{m}$, and $225 \mu \mathrm{m}) .(\mathrm{g}, \mathrm{h})$ Photographs of the device in different configurations, enabling various uses. Adapted with permission from Nat Commun 2013;4:1633/1-10 [114]. Copyright @ 2013 McMillan Publishers Ltd. 
Published in Progress in Polymer Science 43, 48-95, doi: 10.1016/j.progpolymsci.2014.10.001 (2015).

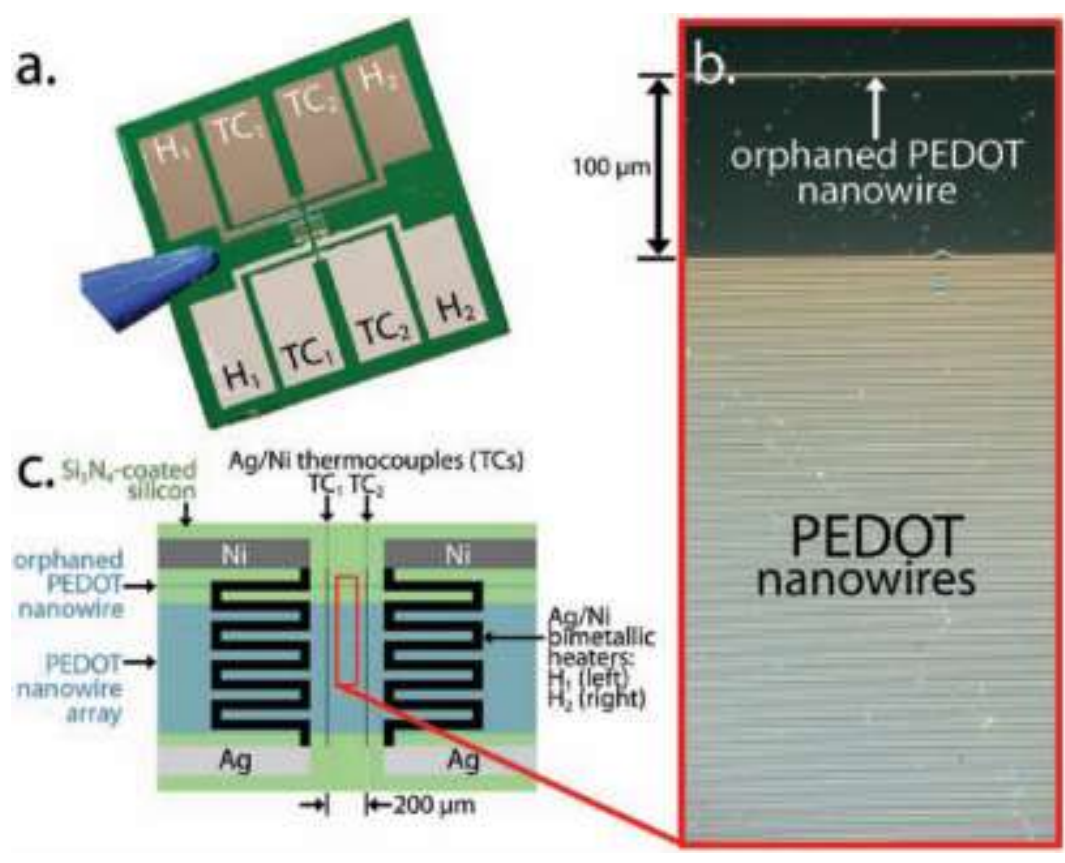

Fig. 34 Thermoelectric PEDOT nanowires. (a) Chip used for measuring thermoelectric figures of merit in PEDOT nanowires. H1, H2: integrated heaters. TC1, TC2: thermocouples. (b) Dark-field optical micrograph of the central region of the chip. (c) Overall device layout. Reproduced with permission from Nano Lett 2011;11:125-31 [325]. Copyright $@ 2011$ American Chemical Society.

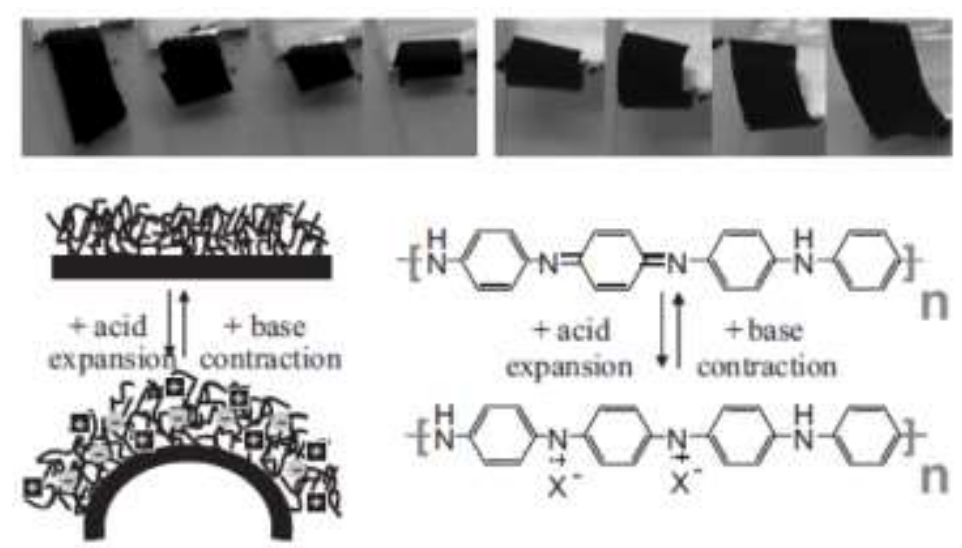

Fig. 35 Actuation of flash-welded PAN nanofibers. The top panels show the sequence of actuation following exposure of nanofibers to camphorsulfonic acid (left), and the reverse process following washing in water and exposure to sodium hydroxide (right). The whole actuation and the reverse process shown in the photographs last $20 \mathrm{~s}$. Bottom panels: schematics illustrating the actuation mechanisms in the asymmetric, flash-welded film of nanofibers upon acid/base exposure. Reproduced with permission from Adv Mater 2008;20:155-8 [334]. Copyright @ 2008 Wiley-VCH Verlag GmbH \& Co. KGaA. 


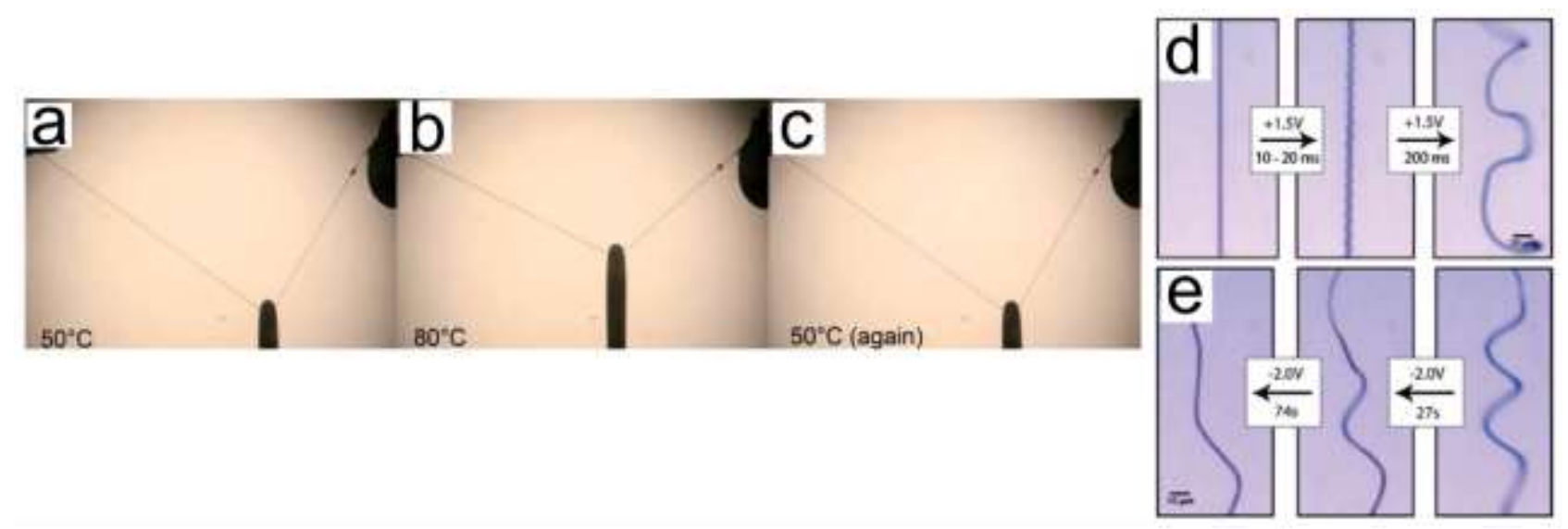

Fig. 36 Various actuators based on polymer micro- and nanofibers. (a-c) Temperature-based control. Photographs of a liquid crystalline elastomer filament glued in between two static substrates and undergone controlled heating. The weight clipped in the middle of the filament highlights the actuation of the filament which is induced by temperature changes. Adapted with permission from Soft Matter 2011;7:3730-4 [338]. Copyright (C) 2011 The Royal Society of Chemistry. (d, e) Oxidized electrospun fibers made of alkoxysilane-functionalized polyferrocenylsilane, rapidly electroactuated (d) and released (e) following the application of $+1.5 \mathrm{~V}$ and $-2.0 \mathrm{~V}$, respectively. Adapted with permission from J Am Chem Soc 2010;132:3236-7 [341]. Copyright @ 2010 American Chemical Society. 